\title{
Supporting information for Enantioselective Organocatalyzed Bromolactonizations: Applications in Natural Product Synthesis
}

Marius Aursnes, Jørn E. Tungen and Trond V. Hansen*

School of Pharmacy, Department of Pharmaceutical Chemistry, University of Oslo, PO Box 1068 Blindern, $N$ 0316 Oslo, Norway

*E-mail: t.v.hansen@farmasi.uio.no

Table of Contents:

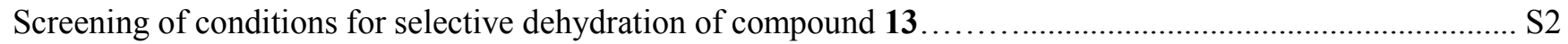

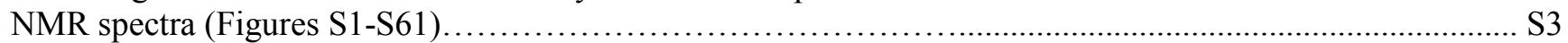

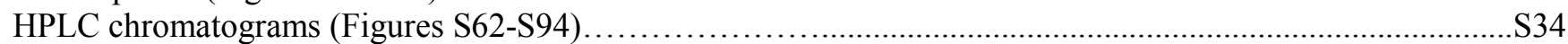

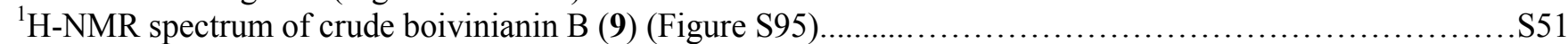

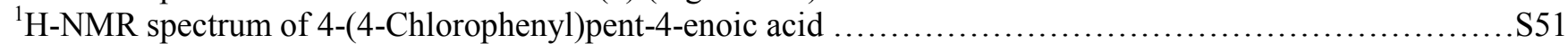

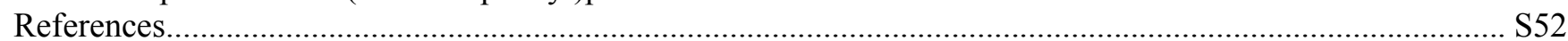


Table S-1 Screening of conditions for selective dehydration of compound $\mathbf{1 3}$

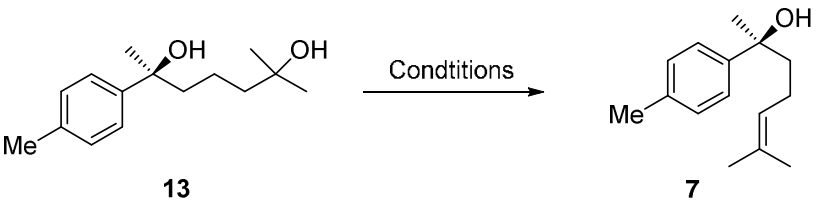

\begin{tabular}{|c|c|c|}
\hline entry & Conditions & $\begin{array}{c}\text { Small scale } \\
\text { yield }(\%)\end{array}$ \\
\hline 1 & DMAP, $\mathrm{MsCl}, \mathrm{CH}_{2} \mathrm{Cl}_{2}$ & 14 \\
\hline 2 & Pyridine, $\mathrm{MsCl}, \mathrm{CH}_{2} \mathrm{Cl}_{2}$ & 5 \\
\hline 3 & $\mathrm{DBU}, \mathrm{MsCl}, \mathrm{CH}_{2} \mathrm{Cl}_{2}$ & 34 \\
\hline 4 & $\mathrm{NEt}_{3}, \mathrm{MsCl}$ & 7 \\
\hline 5 & DBU, $\mathrm{TsCl}, \mathrm{CH}_{2} \mathrm{Cl}_{2}$ & - \\
\hline 6 & DMAP, DBU, TsCl, $\mathrm{CH}_{2} \mathrm{Cl}_{2}$ & - \\
\hline 7 & Martin sulfurane, benzene, $\mathrm{rt}$ & 23 \\
\hline 8 & Burgess reagent, benzene, $\mathrm{rt}$ & - \\
\hline 9 & $\mathrm{P}_{2} \mathrm{O}_{5}$, benzene & 15 \\
\hline 10 & DBU, 2-propanesulfonyl chloride, $\mathrm{CH}_{2} \mathrm{Cl}_{2}$ & 12 \\
\hline 11 & $\mathrm{POCl}_{3}$, pyridine, $0^{\circ} \mathrm{C}$ & 58 \\
\hline
\end{tabular}

As an alternative to purification by chromatography as outlined in the Experimental Section, the regioisomeric mixture could be isomerized to the desired isomer, i.e. (-)-gossonorol (7), by the following protocol: The crude material obtained from the above phosphorus oxychloride-mediated dehydration protocol was dissolved in $96 \%$ ethanol $(10 \mathrm{~mL} / \mathrm{mmol}$ material $)$ and $\mathrm{K}_{2} \mathrm{CO}_{3}(45 \mathrm{~mol} \%)$ and $\mathrm{RhCl}_{3} \cdot 3 \mathrm{H}_{2} \mathrm{O}(25 \mathrm{~mol} \%)$ were added. The setup was evacuated and filled with argon $(3 \mathrm{x})$ and refluxed overnight. Next, the reaction mixture was cooled to room temperature, concentrated in vacuo and the material thus obtained was subjected to purification using flash column chromatography on silica (EtOAc/ /heptane, 15:85). 


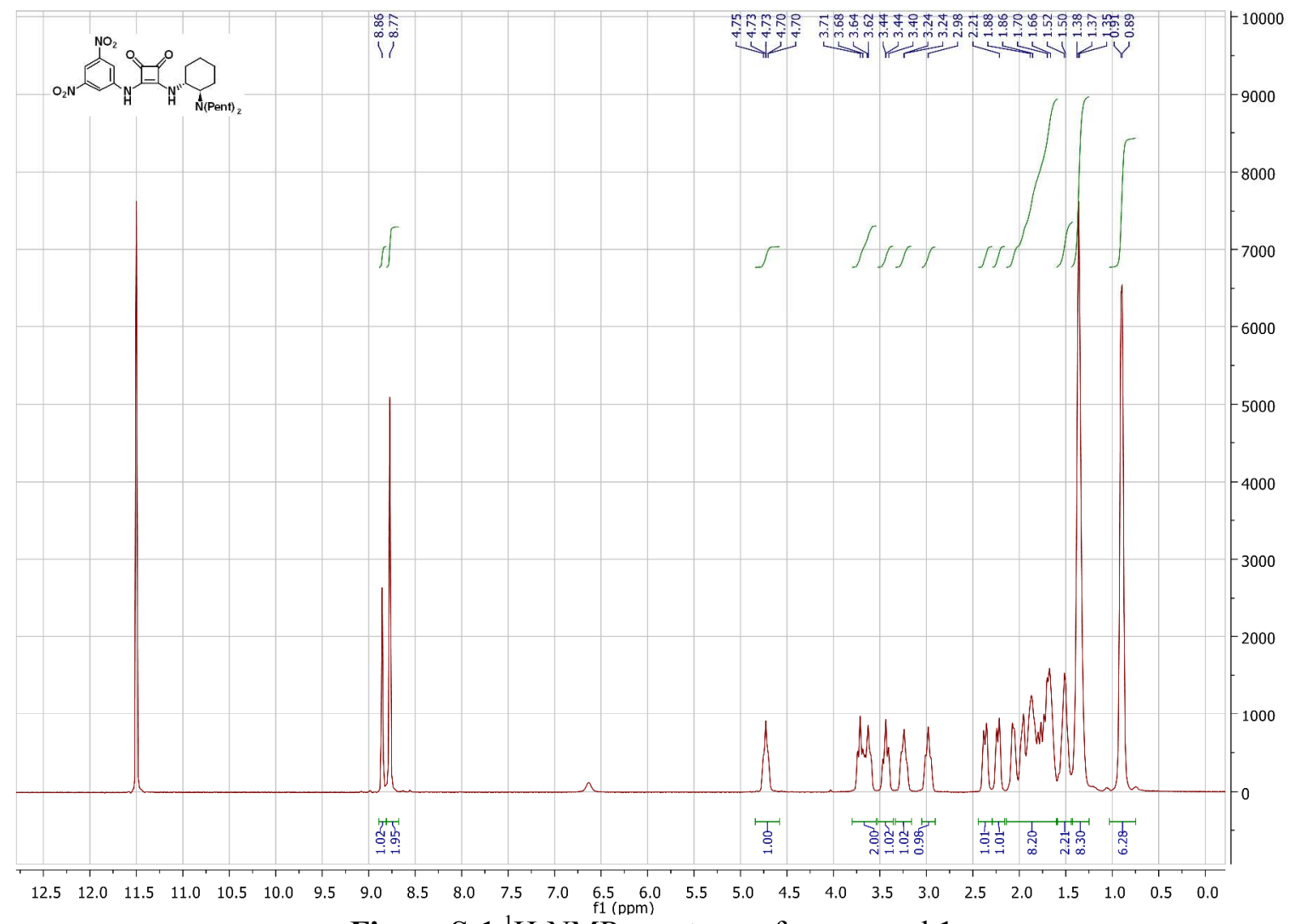

Figure S-1 ${ }^{1}$ H-NMR spectrum of compound 1 a.

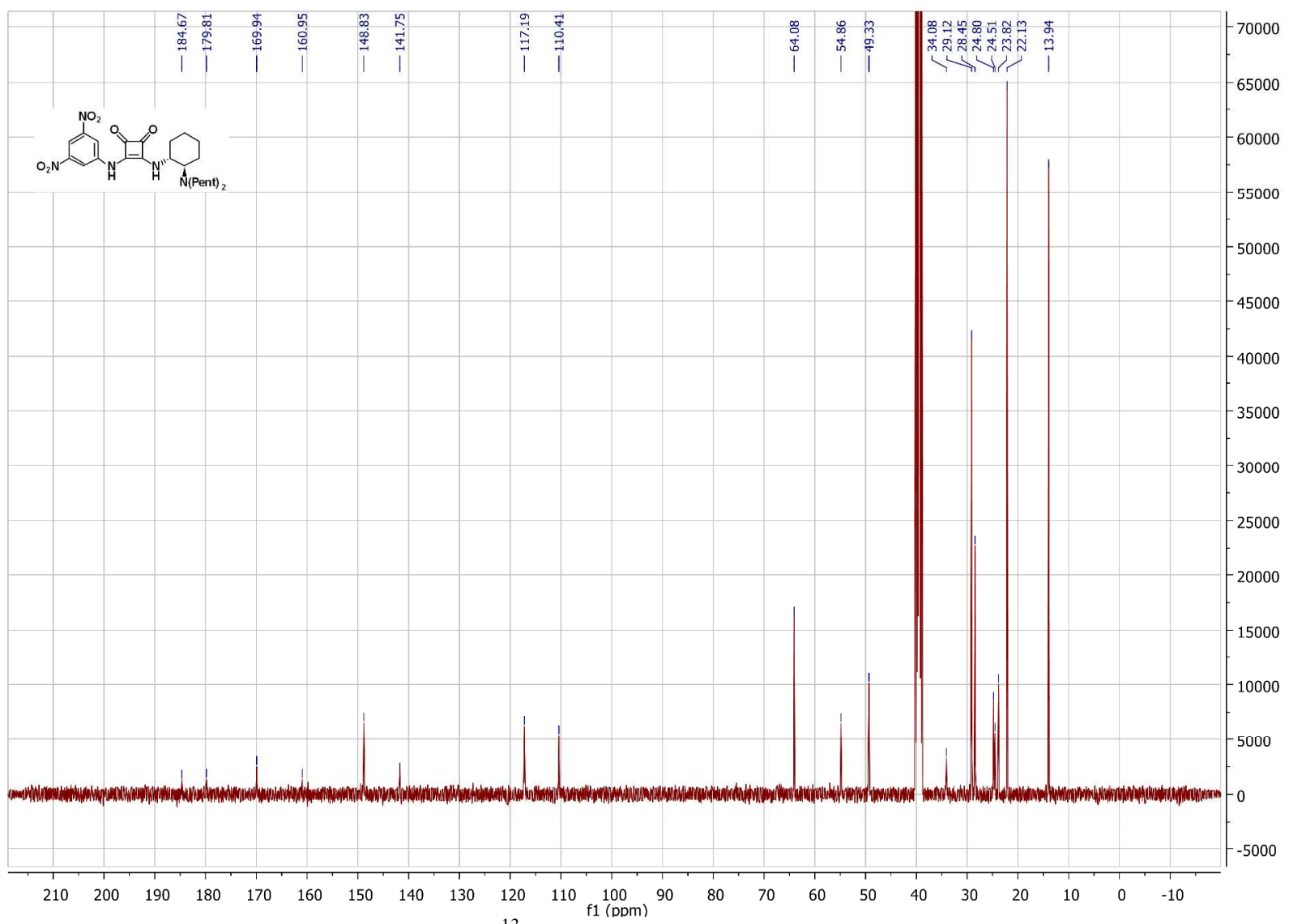

Figure S-2 ${ }^{13} \mathrm{C}-\mathrm{NMR}$ spectrum of compound $\mathbf{1 a}$. 


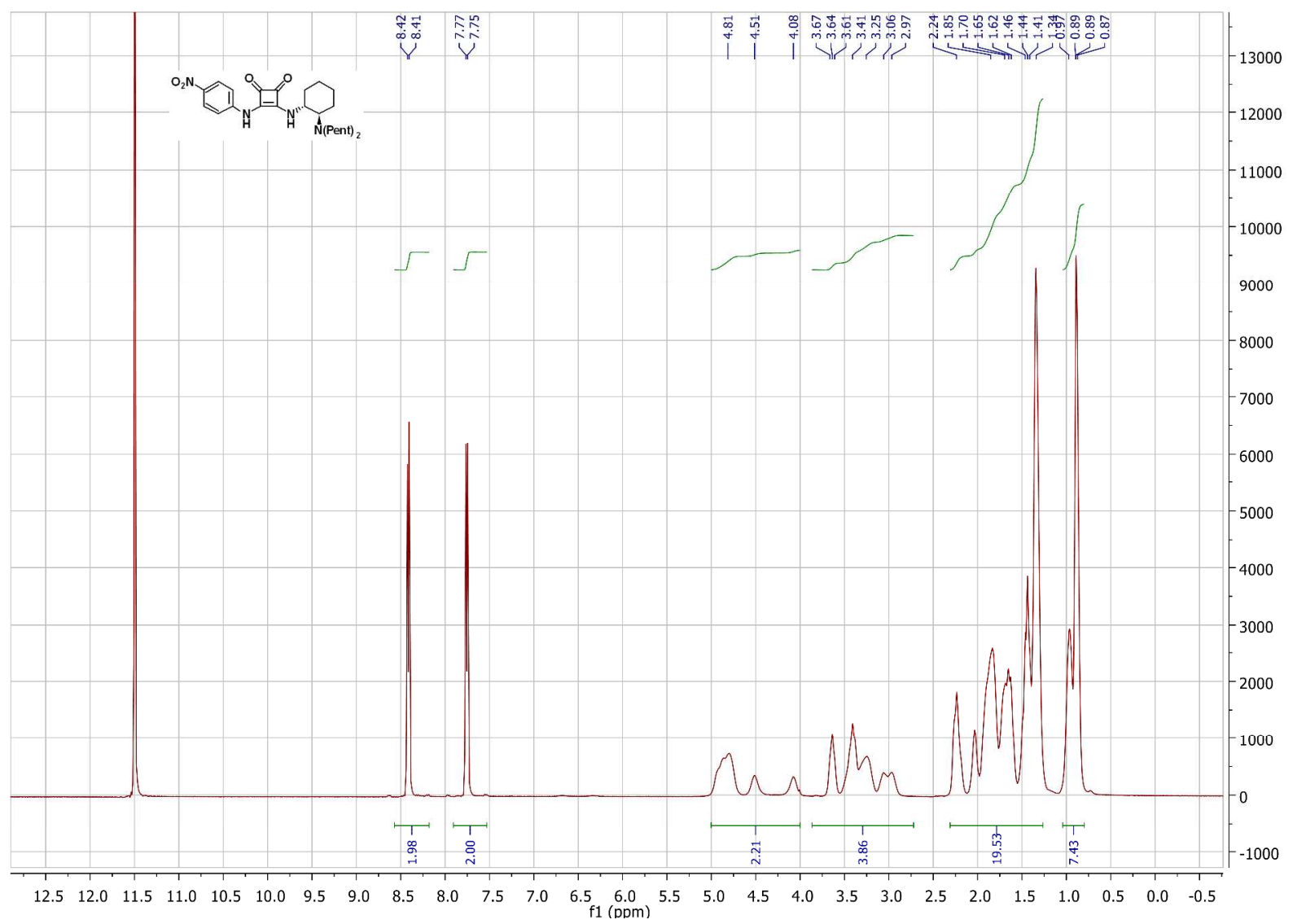

Figure S-3 ${ }^{1}$ H-NMR spectrum of compound $\mathbf{1 c}$.

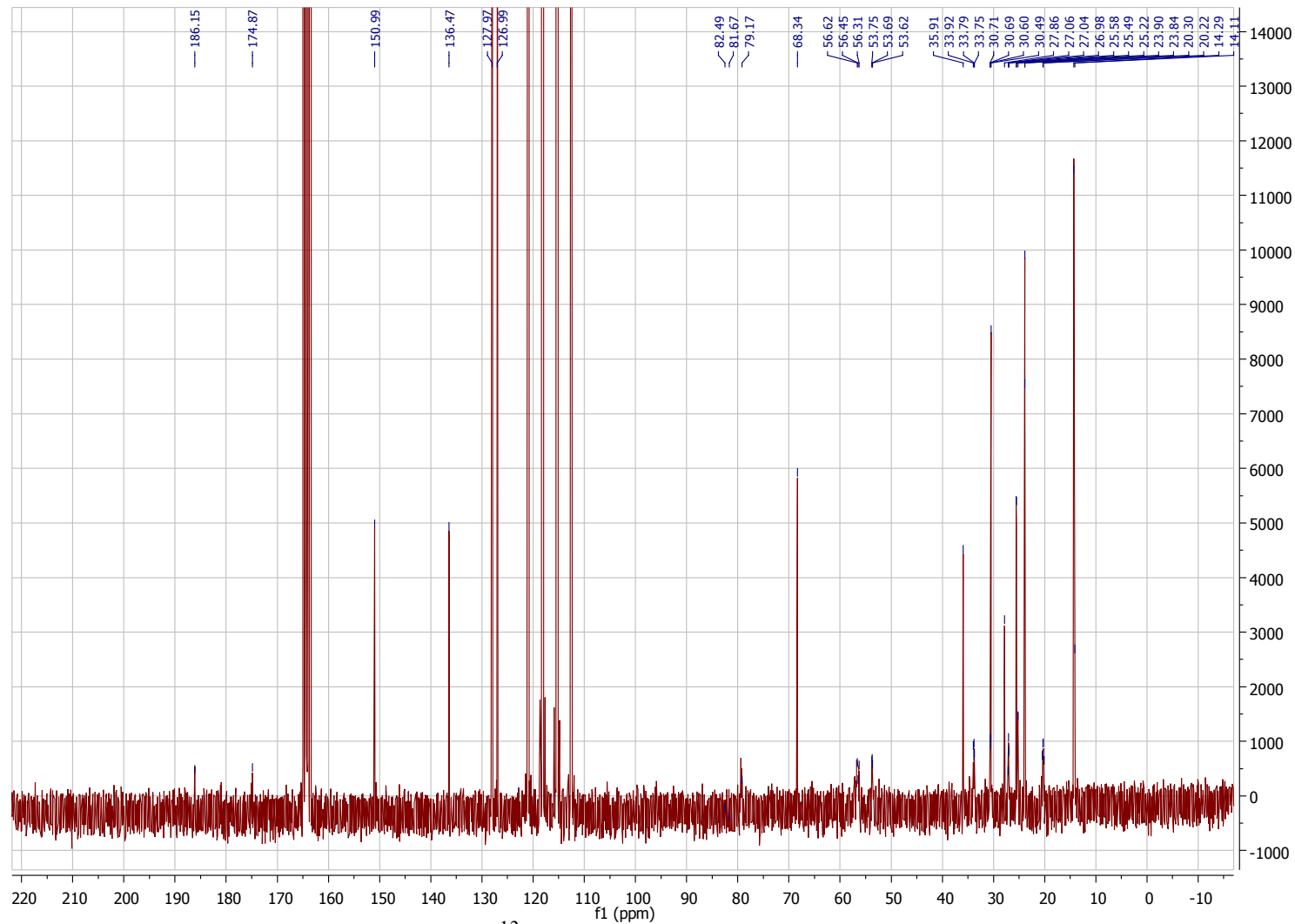

Figure S-4 ${ }^{13} \mathrm{C}-\mathrm{NMR}$ spectrum of compound $\mathbf{1 c}$. 


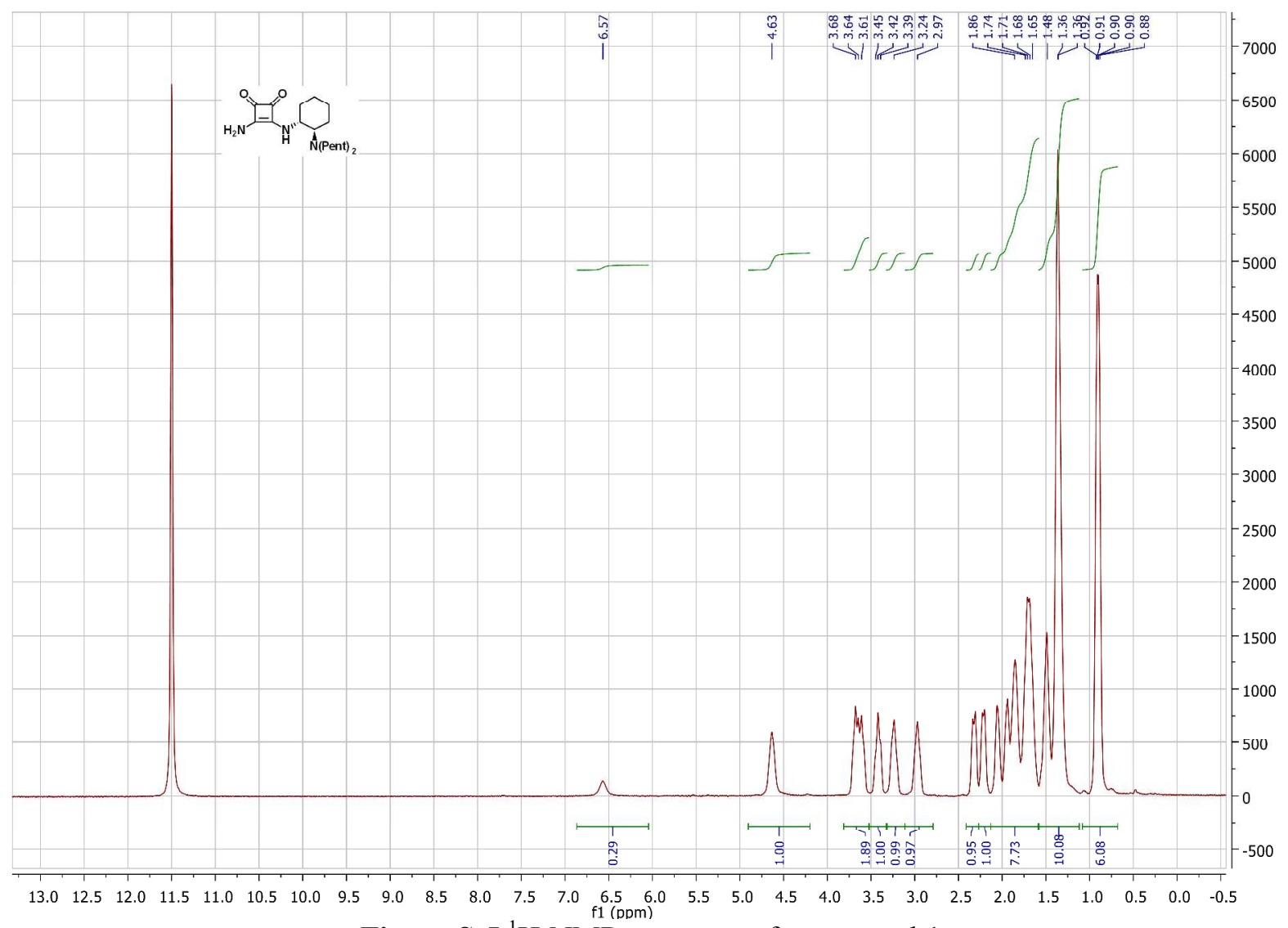

Figure S-5 ${ }^{1} \mathrm{H}-\mathrm{NMR}$ spectrum of compound $\mathbf{1 g}$.

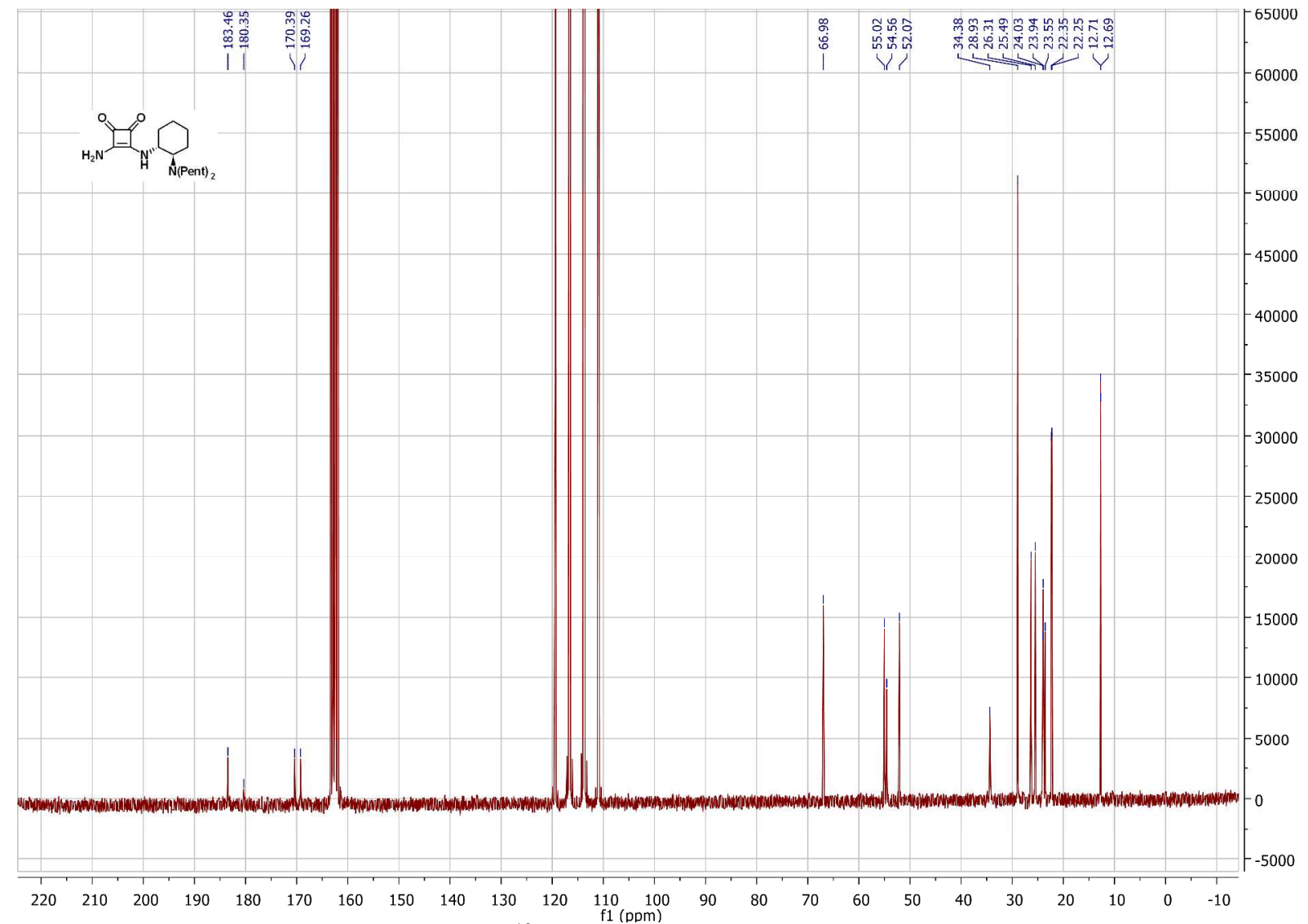

Figure S-6 ${ }^{13} \mathrm{C}-\mathrm{NMR}$ spectrum of compound $\mathbf{1 g}$. 


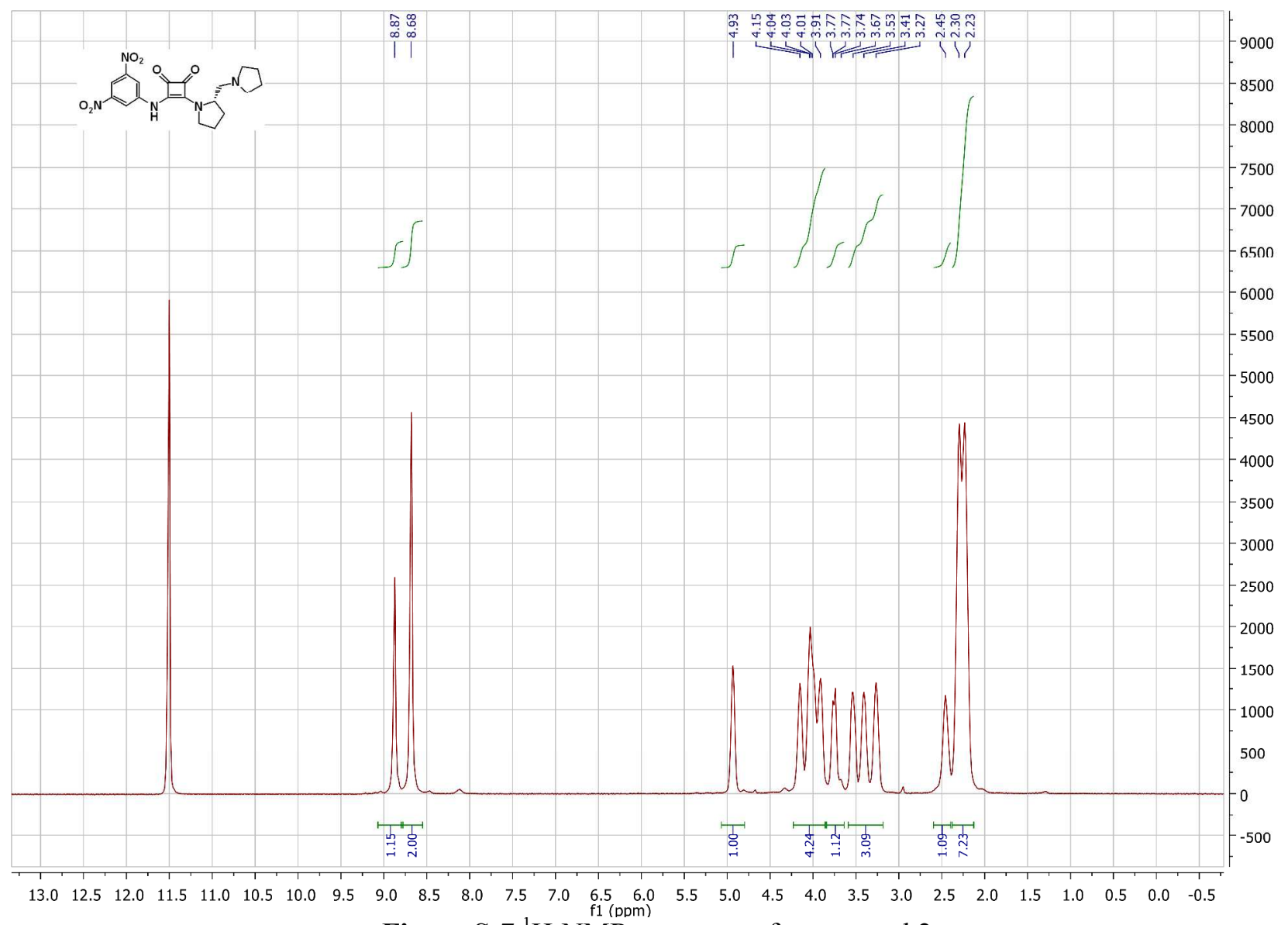

Figure S-7 ${ }^{1} \mathrm{H}-\mathrm{NMR}$ spectrum of compound 2.

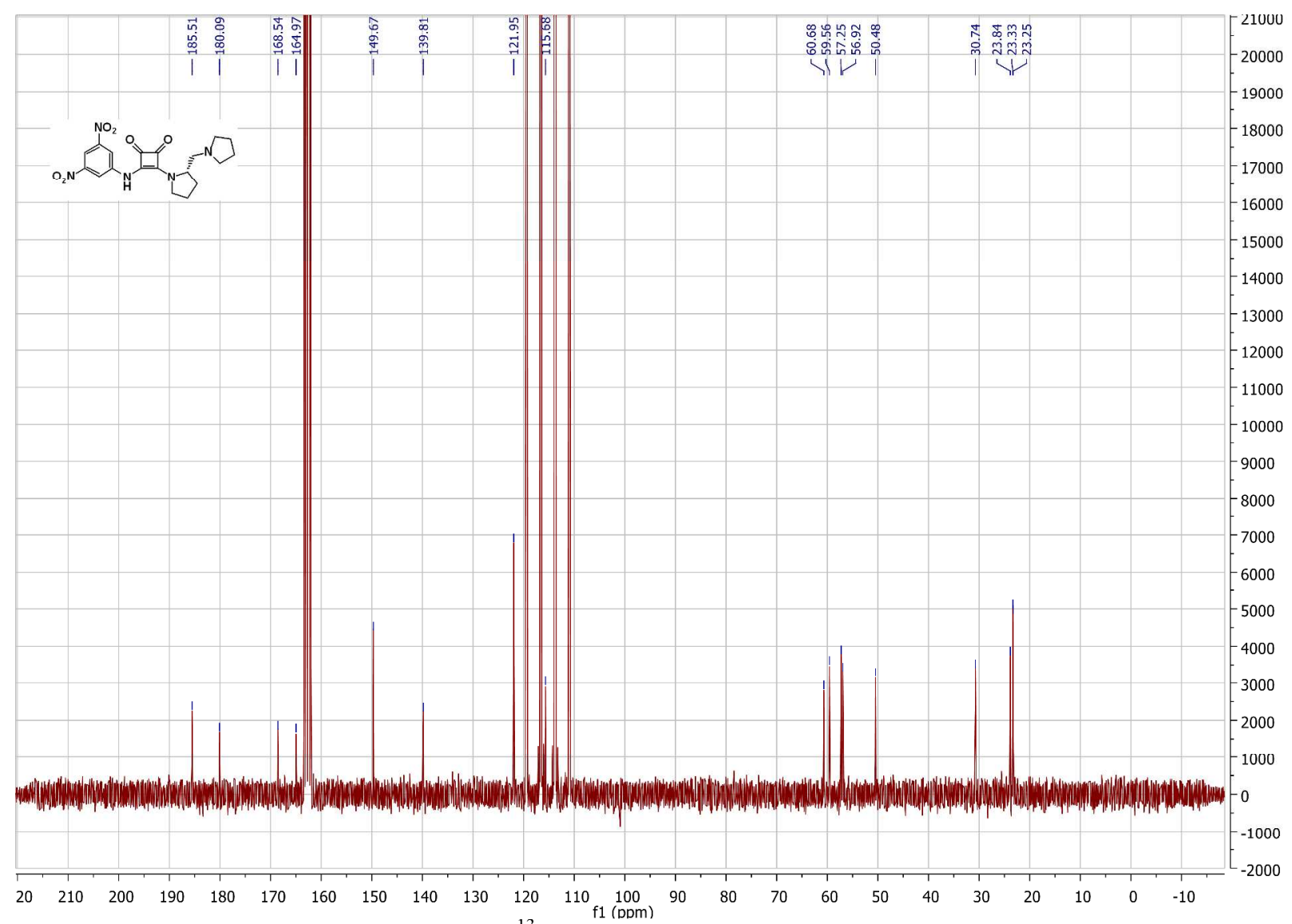

Figure S-8 ${ }^{13} \mathrm{C}-\mathrm{NMR}$ spectrum of compound 2 . 


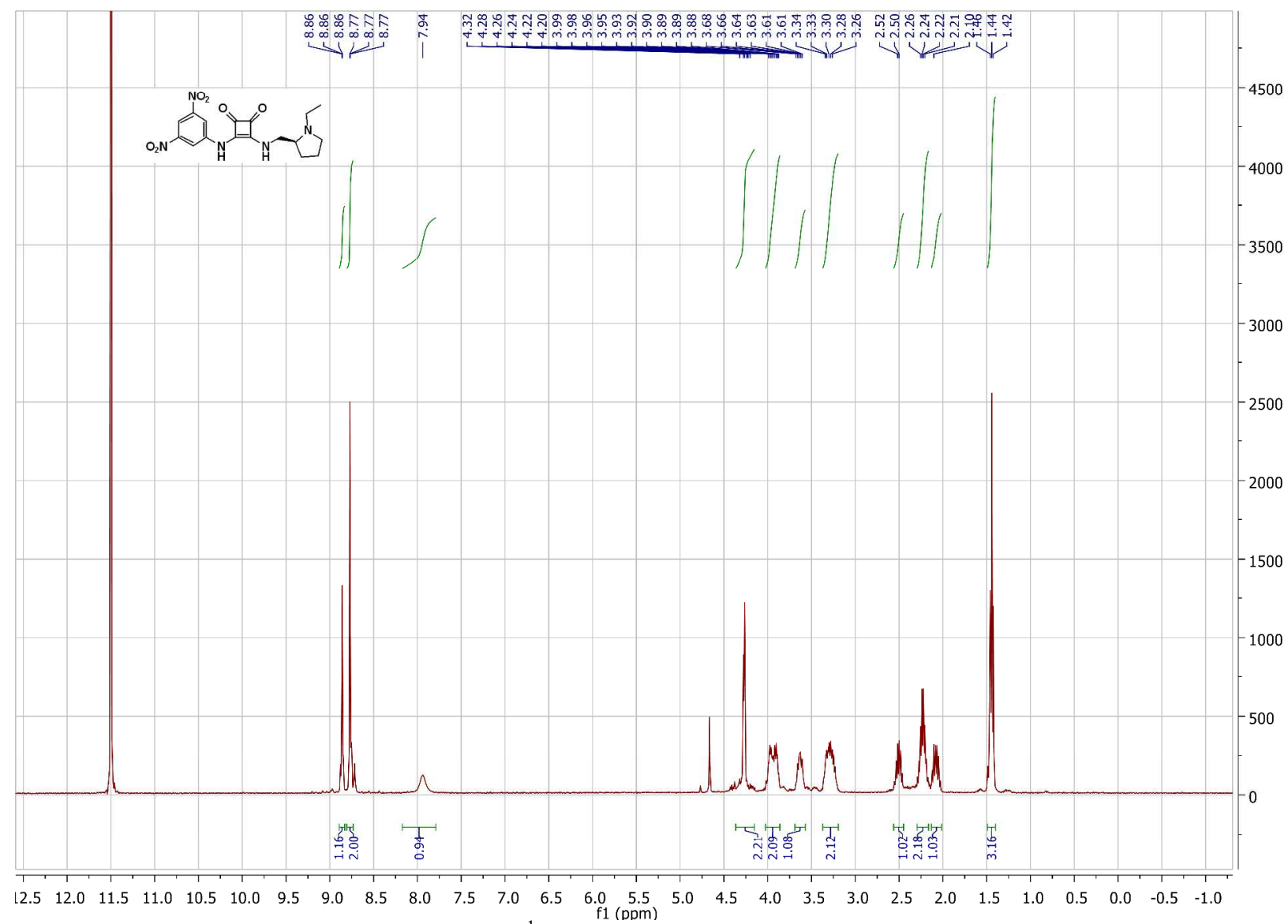

Figure S-9 ${ }^{1} \mathrm{H}-\mathrm{NMR}$ spectrum of compound 3 .

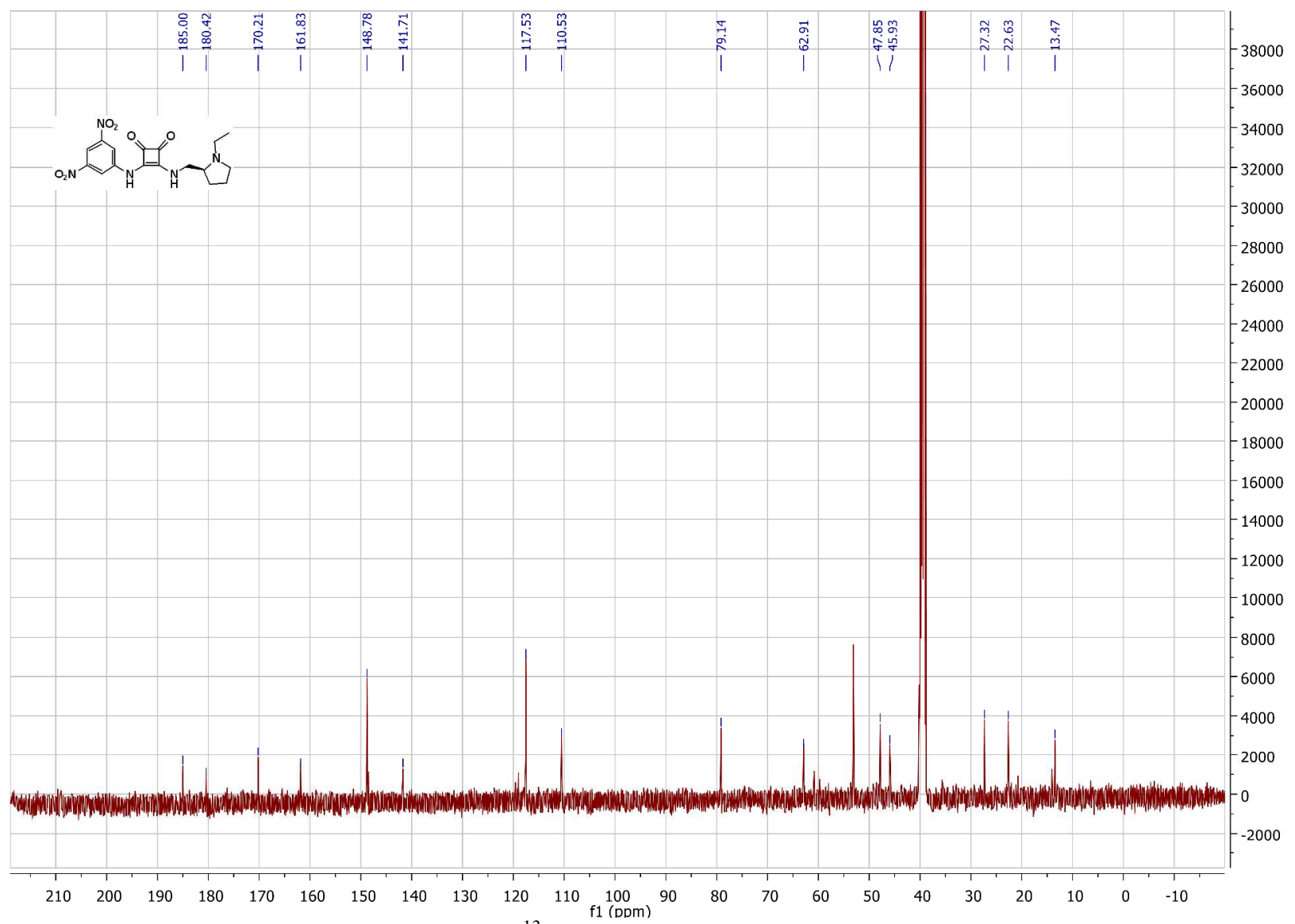

Figure S-10 ${ }^{13} \mathrm{C}$-NMR spectrum of compound 3 . 


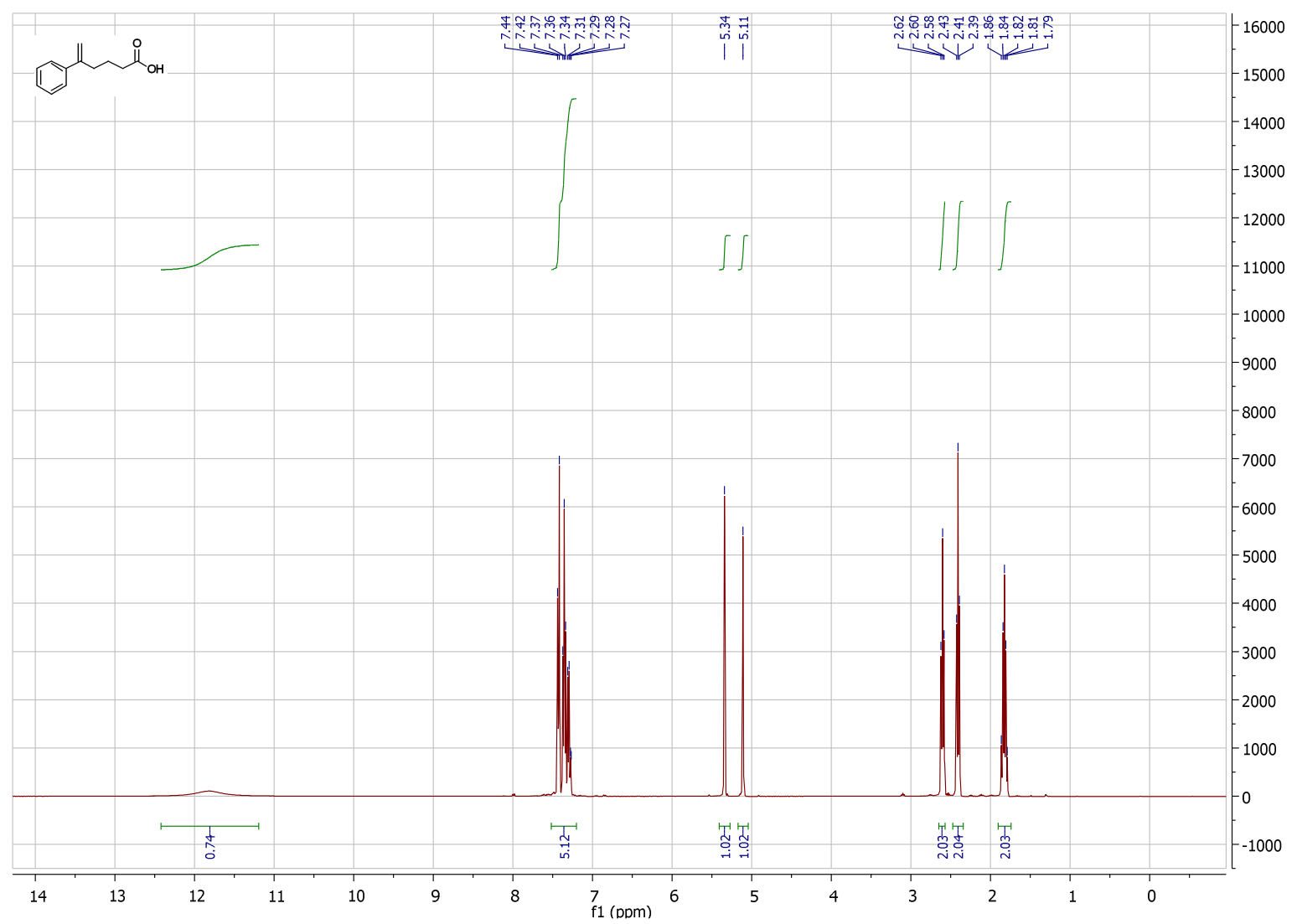

Figure S-11 ${ }^{1}$ H-NMR spectrum of compound 5a.

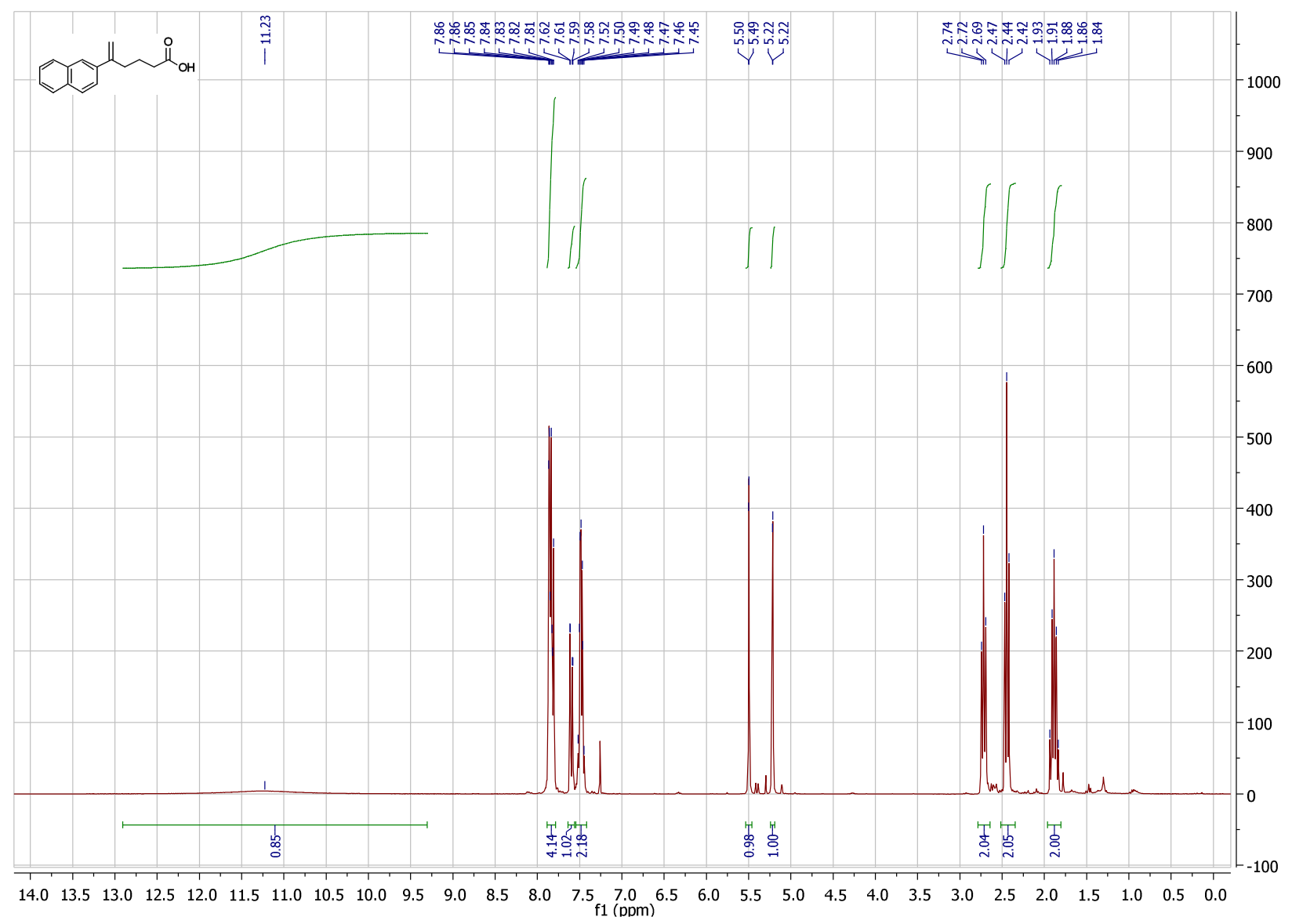

Figure S-12 ${ }^{1} \mathrm{H}-\mathrm{NMR}$ spectrum of compound $\mathbf{5 b}$. 


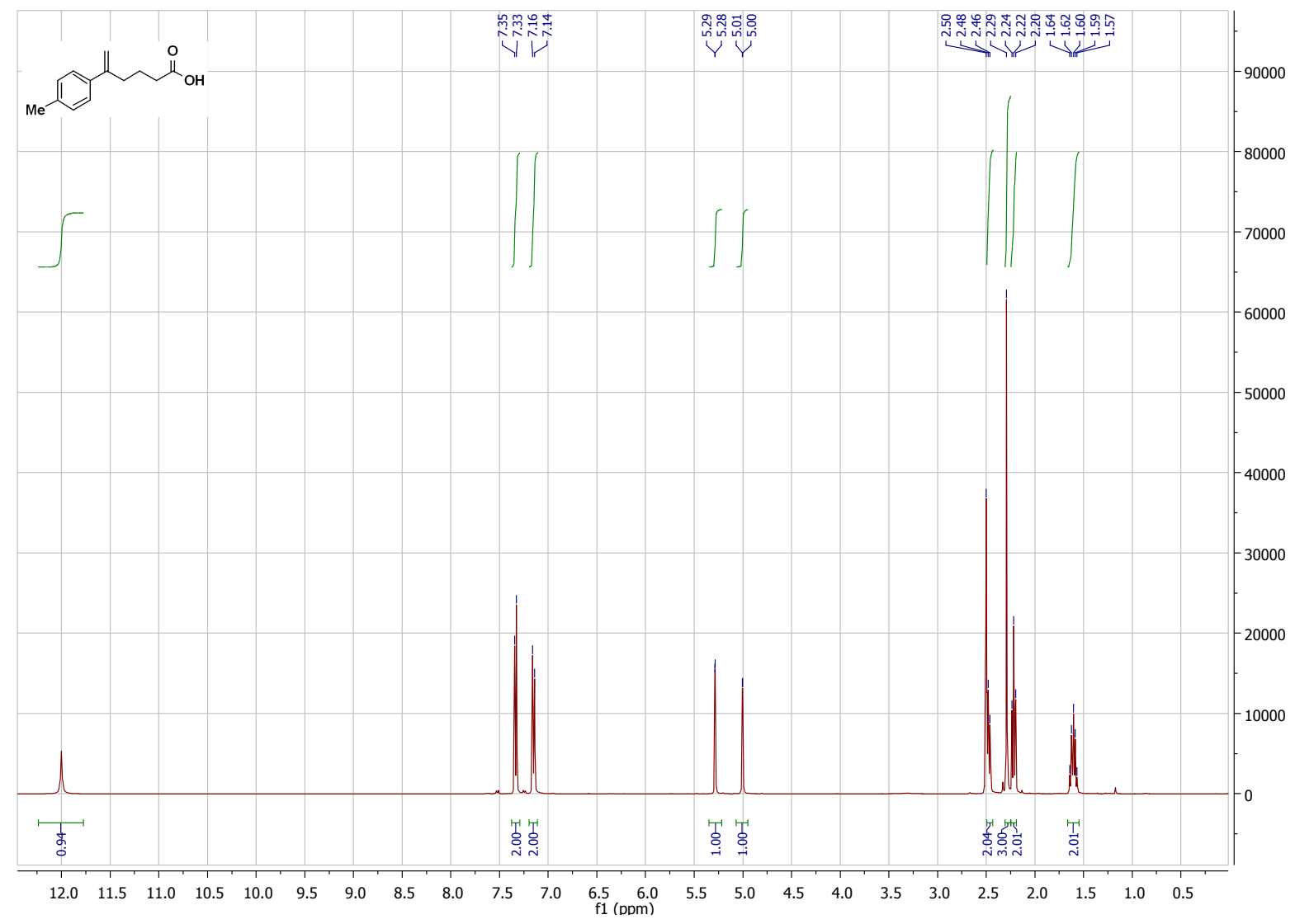

Figure S-13 ${ }^{1}$ H-NMR spectrum of compound $\mathbf{5 c}$.

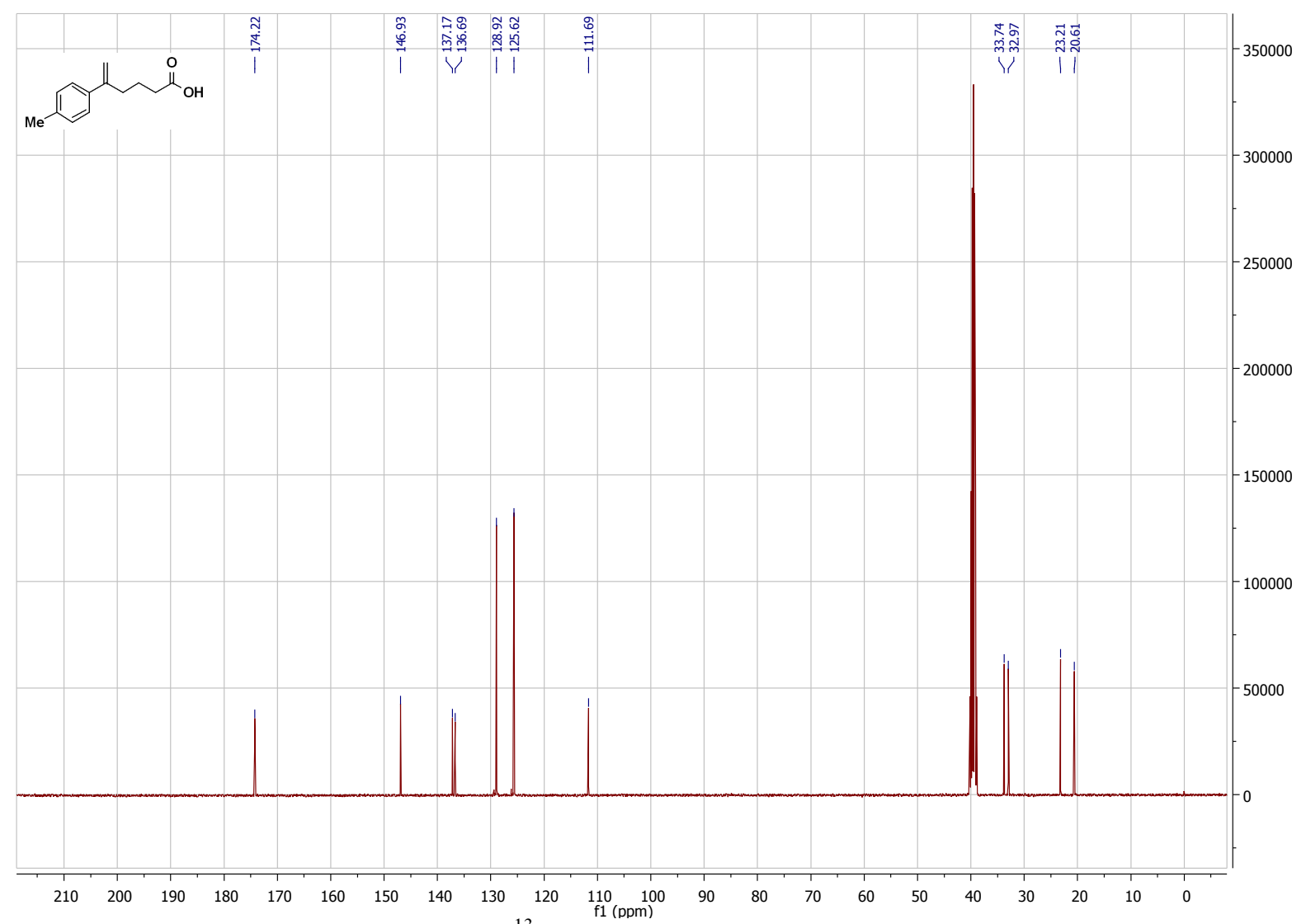

Figure S-14 ${ }^{13} \mathrm{C}$-NMR spectrum of compound $\mathbf{5 c}$. 


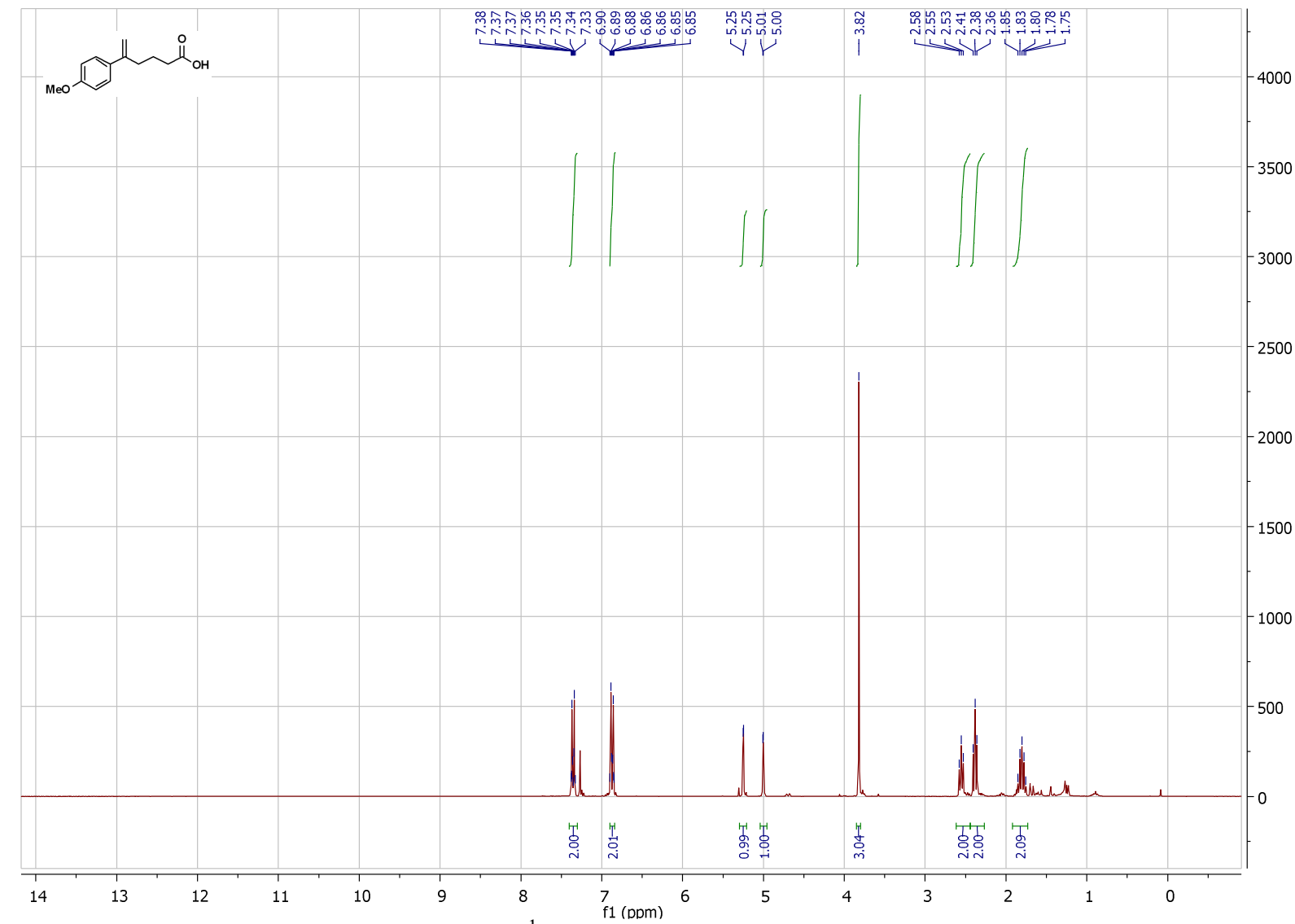

Figure S-15 ${ }^{1}$ H-NMR spectrum of compound $\mathbf{5 d}$.

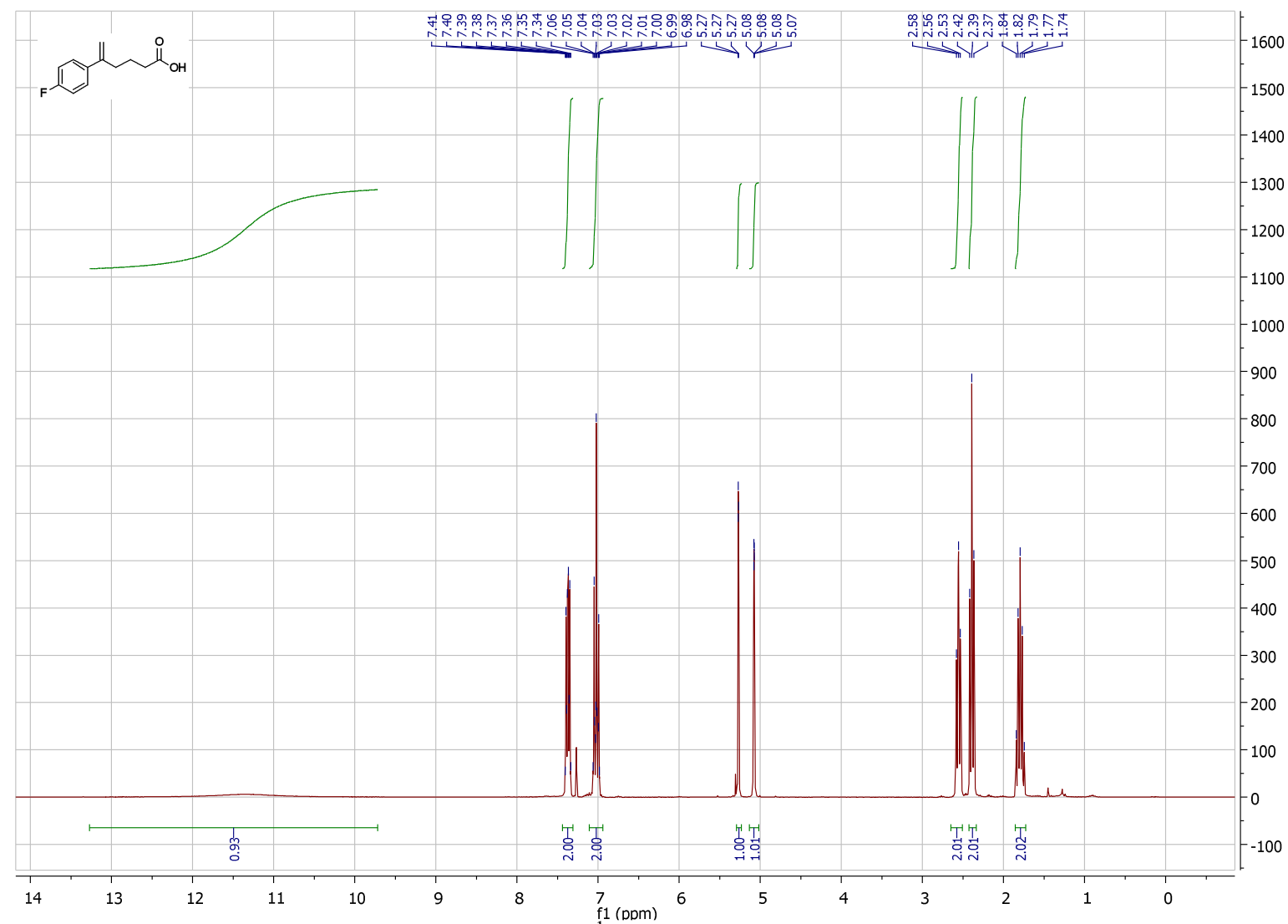

Figure S-16 ${ }^{1} \mathrm{H}-\mathrm{NMR}$ spectrum of compound $\mathbf{5 e}$. 


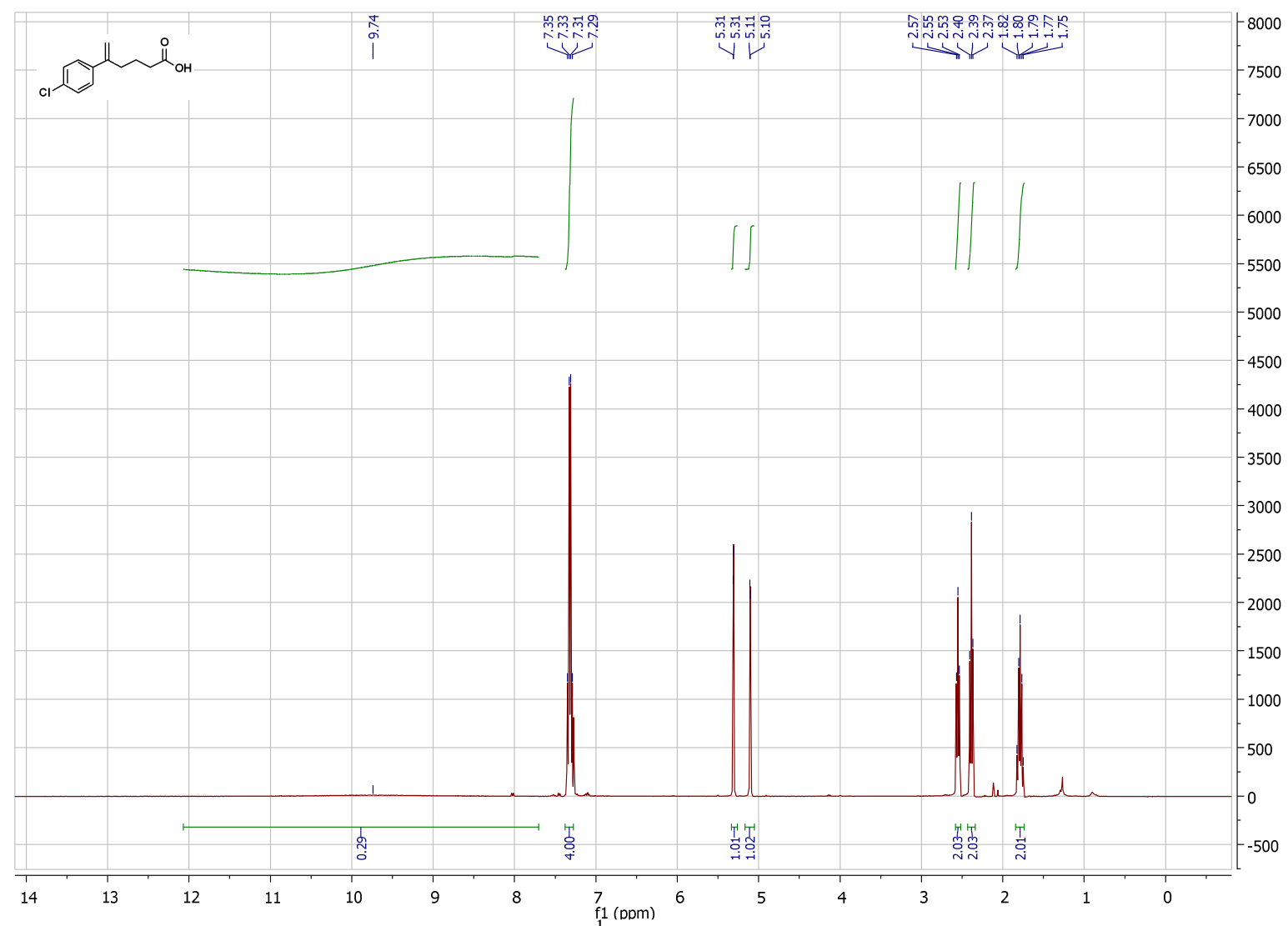

Figure S-17 ${ }^{1} \mathrm{H}-\mathrm{NMR}$ spectrum of compound $\mathbf{5 f}$.

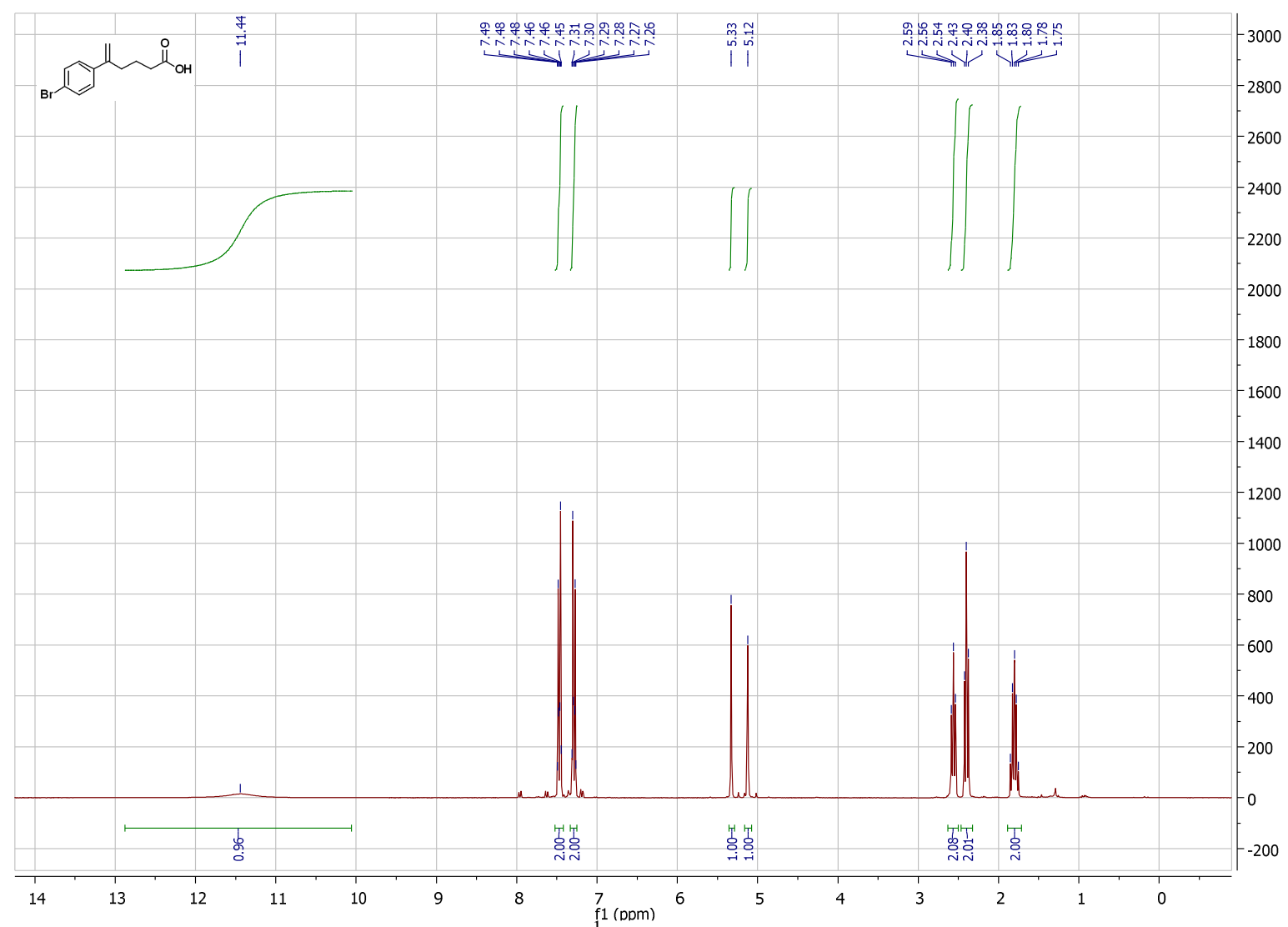

Figure S-18 ${ }^{1} \mathrm{H}-\mathrm{NMR}$ spectrum of compound $\mathbf{5 g}$. 


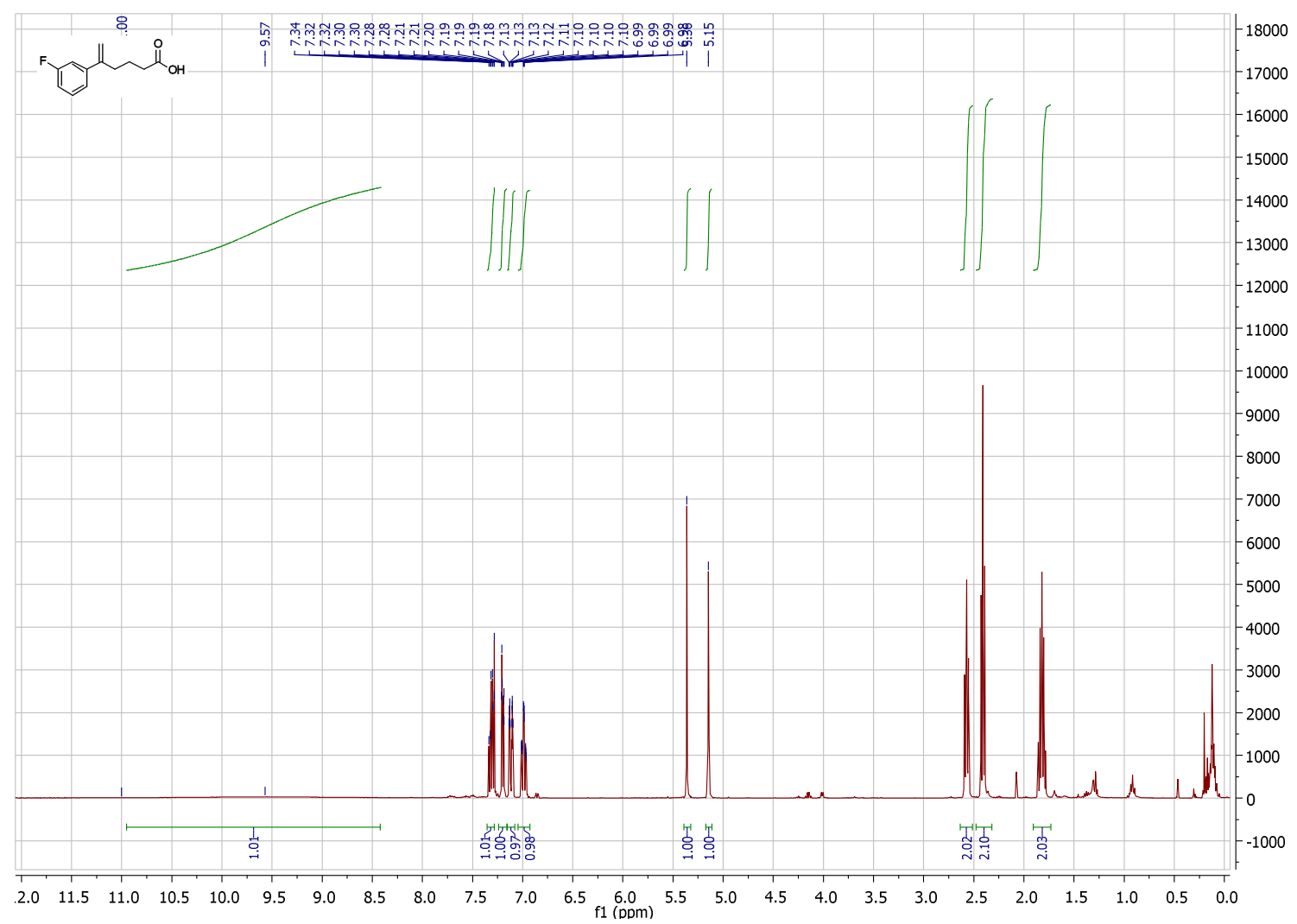

Figure S-19 ${ }^{1}$ H-NMR spectrum of compound $\mathbf{5 h}$.

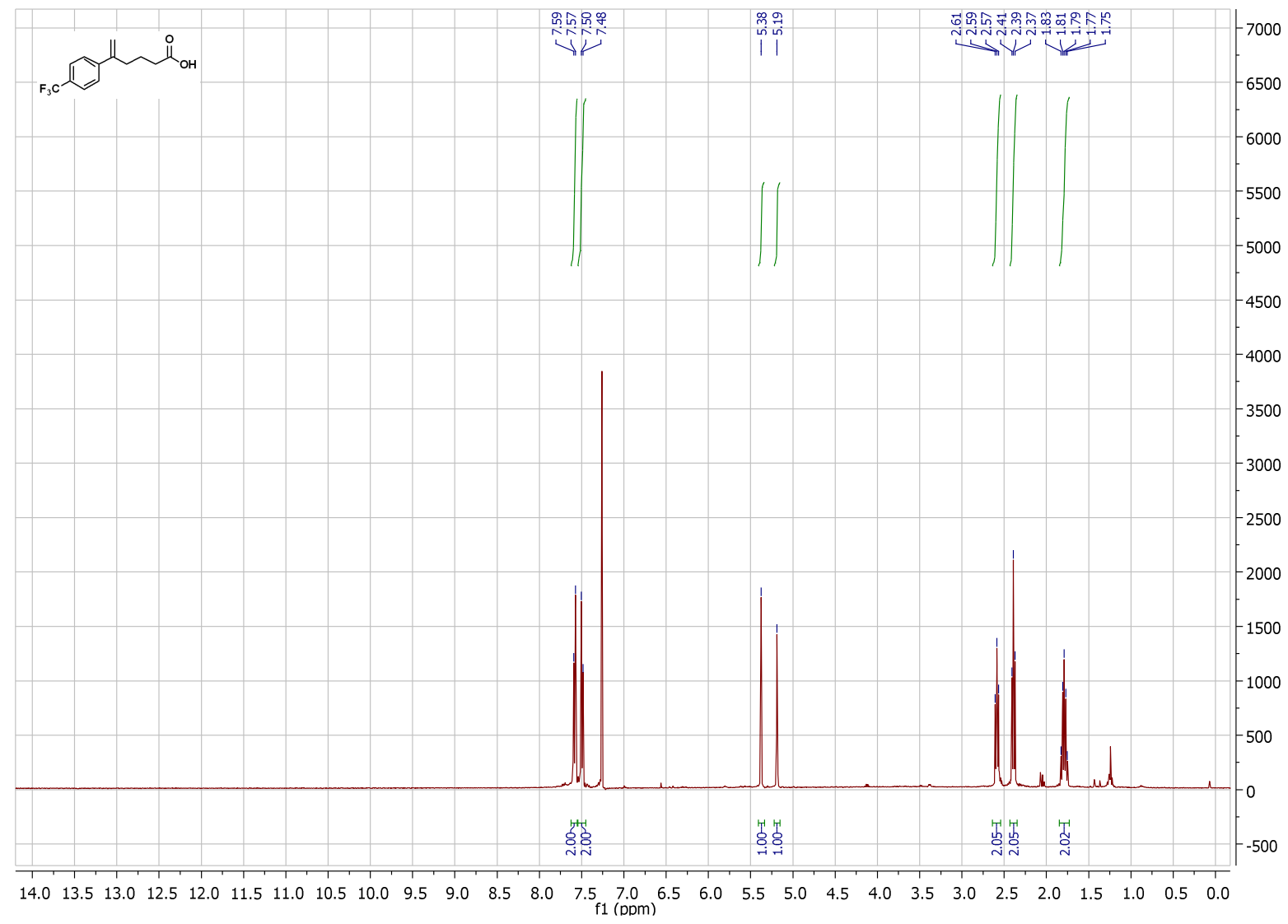

Figure S-20 ${ }^{1} \mathrm{H}-\mathrm{NMR}$ spectrum of compound $\mathbf{5 i}$. 


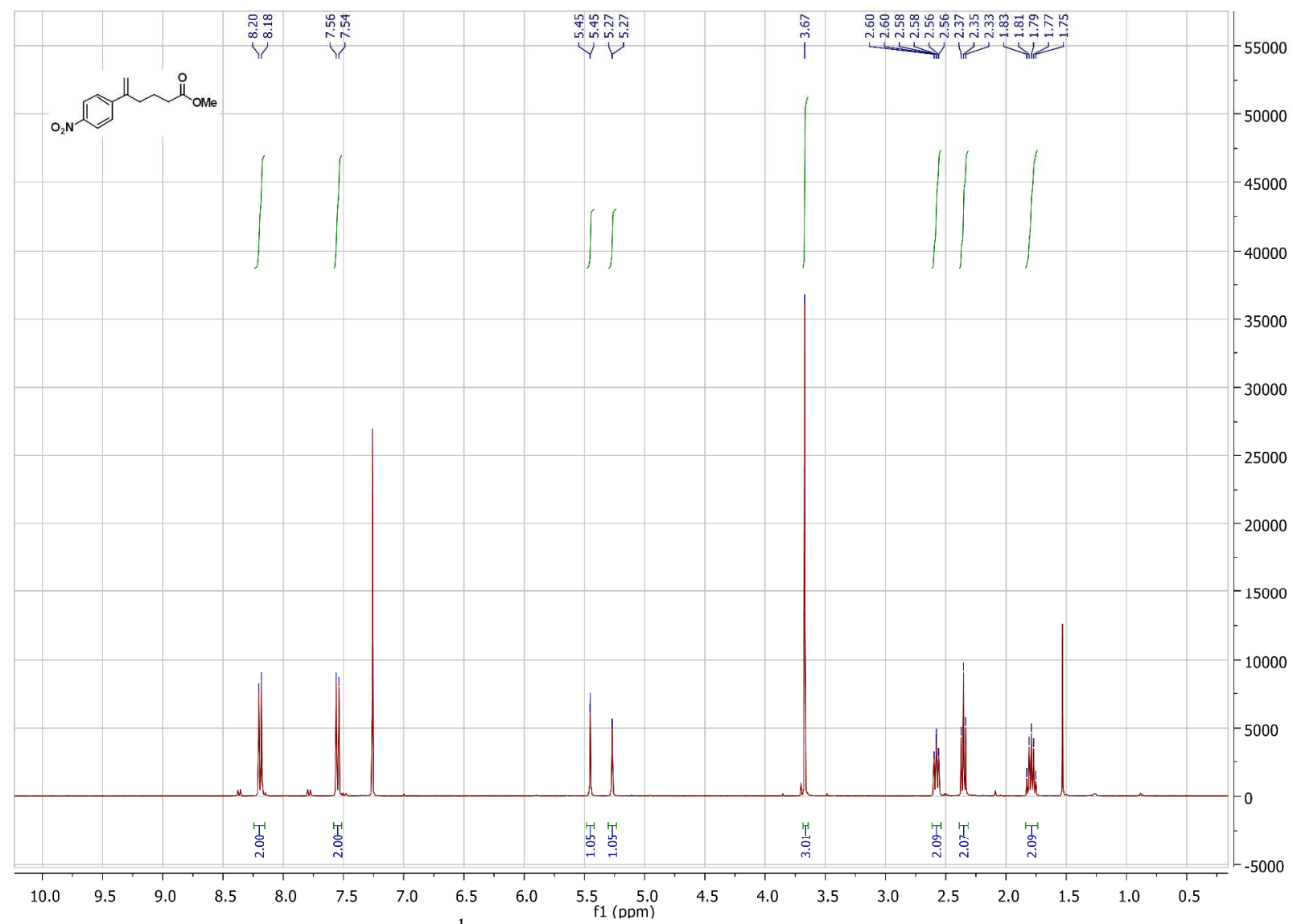

Figure S-21 ${ }^{1}$ H-NMR spectrum of the methyl ester of compound $\mathbf{5 j}$.

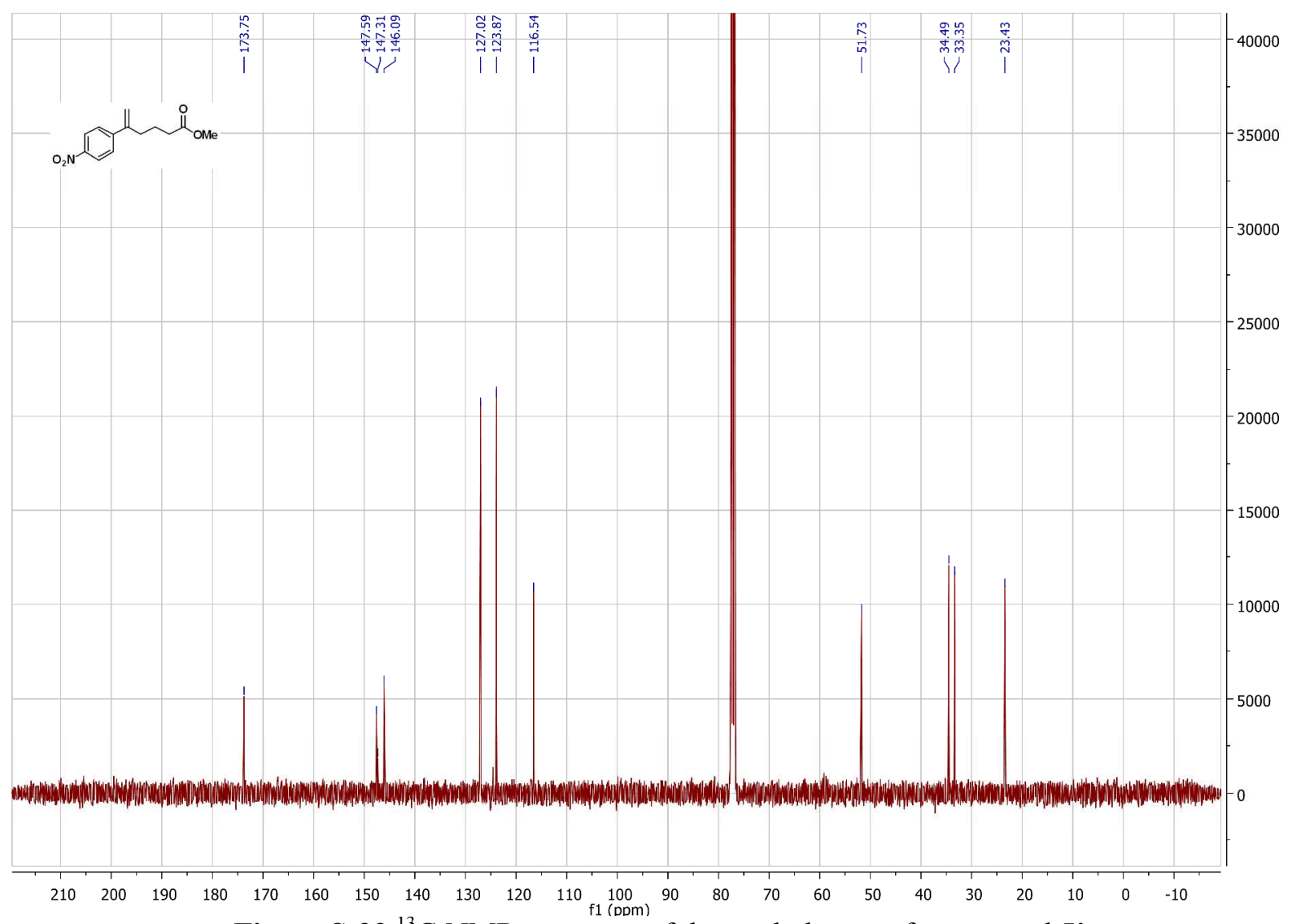

Figure S-22 ${ }^{13} \mathrm{C}-\mathrm{NMR}$ spectrum of the methyl ester of compound $\mathbf{5 j}$. 


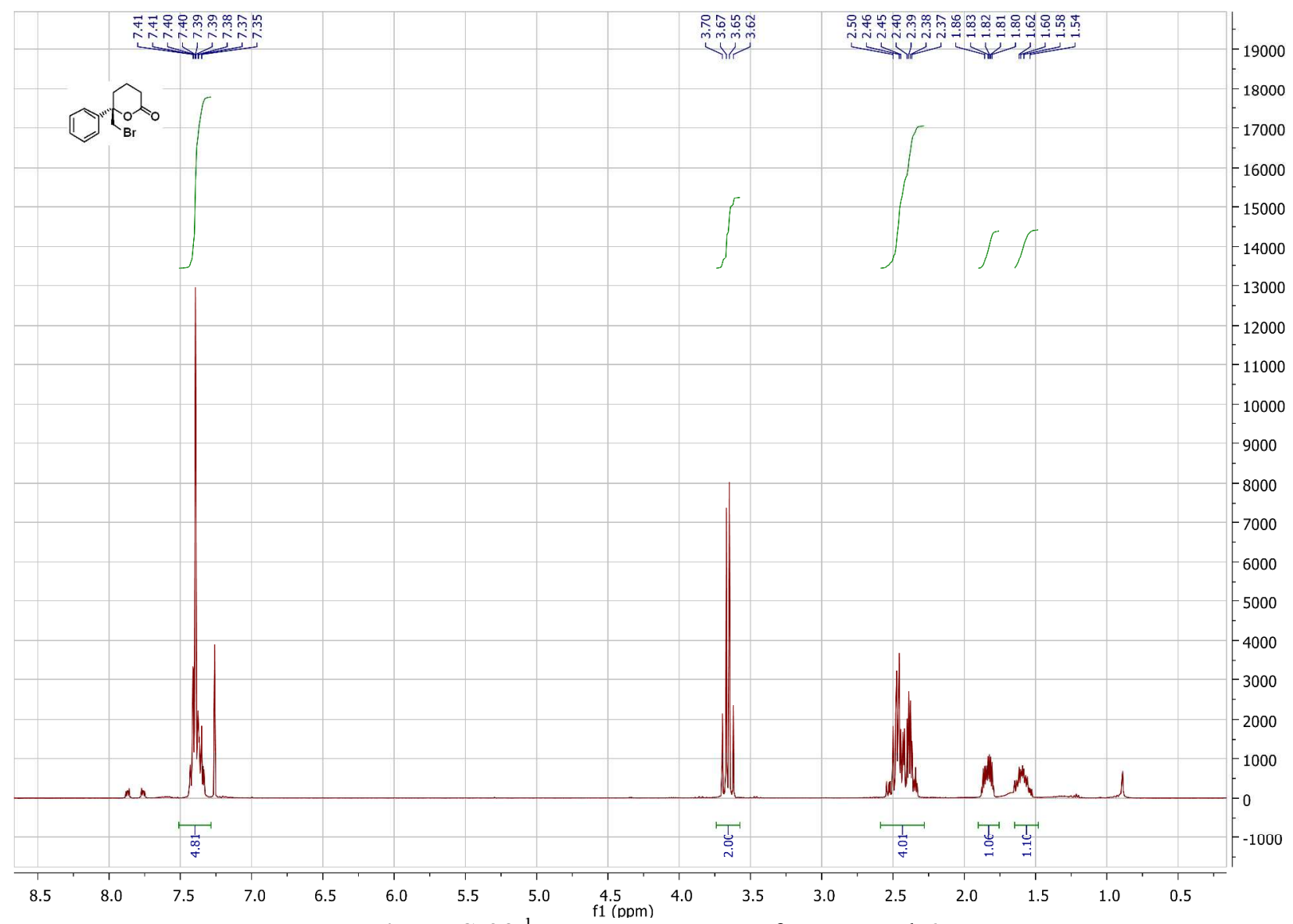

Figure S-23 ${ }^{1}$ H-NMR spectrum of compound $6 \mathbf{a}$.

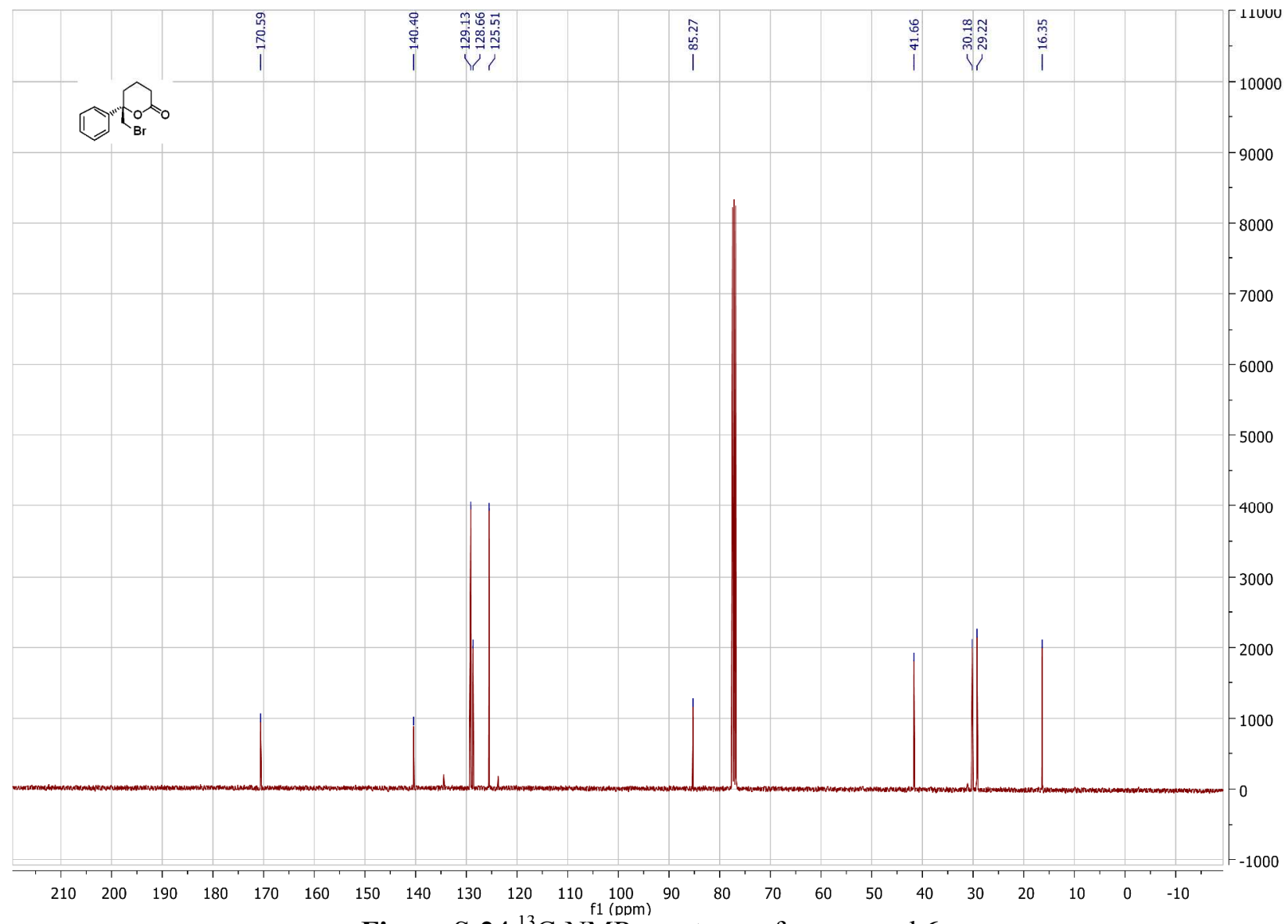

Figure S-24 ${ }^{13} \mathrm{C}$-NMR spectrum of compound $6 \mathbf{a}$. 


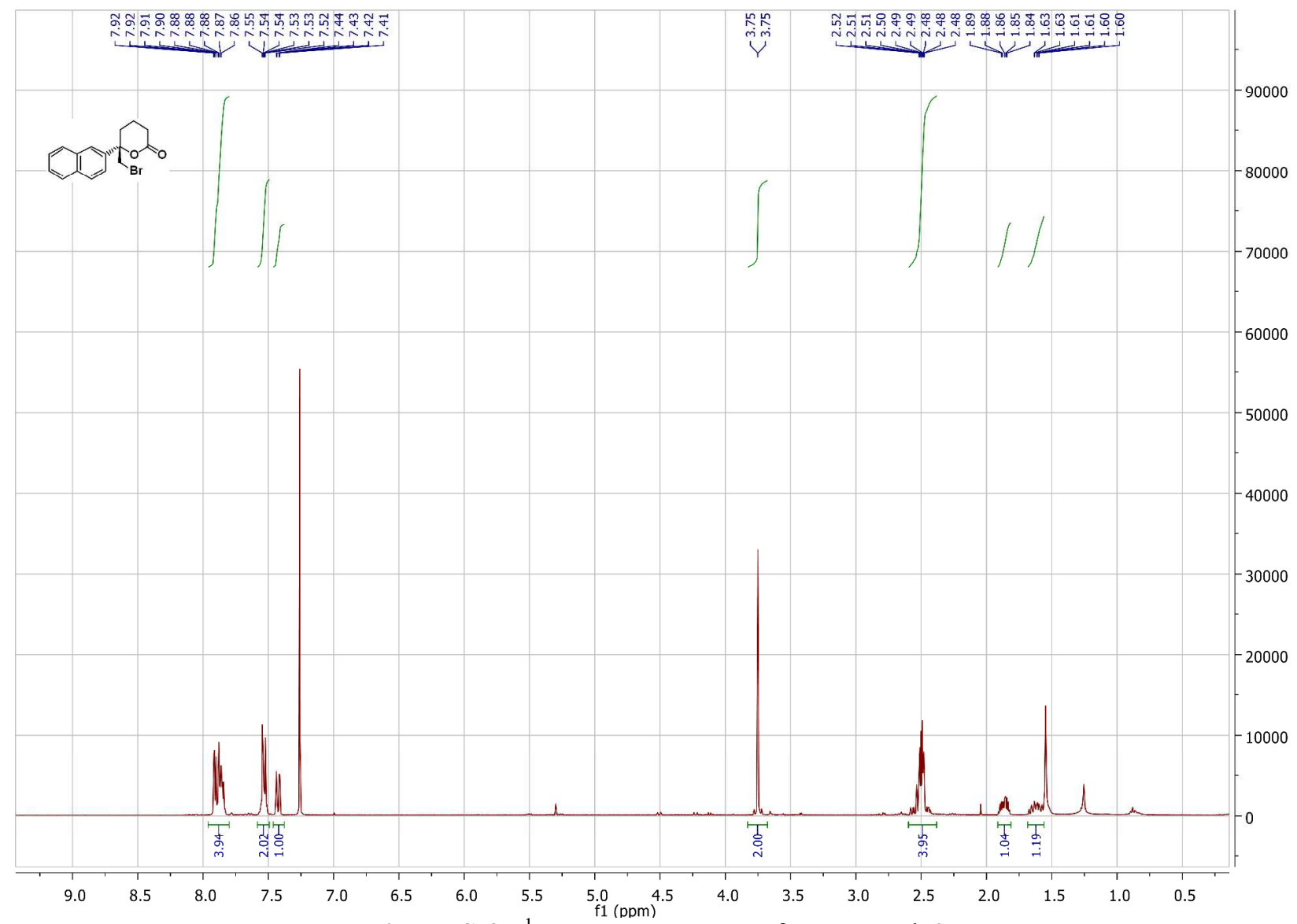

Figure S-25 ${ }^{1}$ H-NMR spectrum of compound $\mathbf{6 b}$.

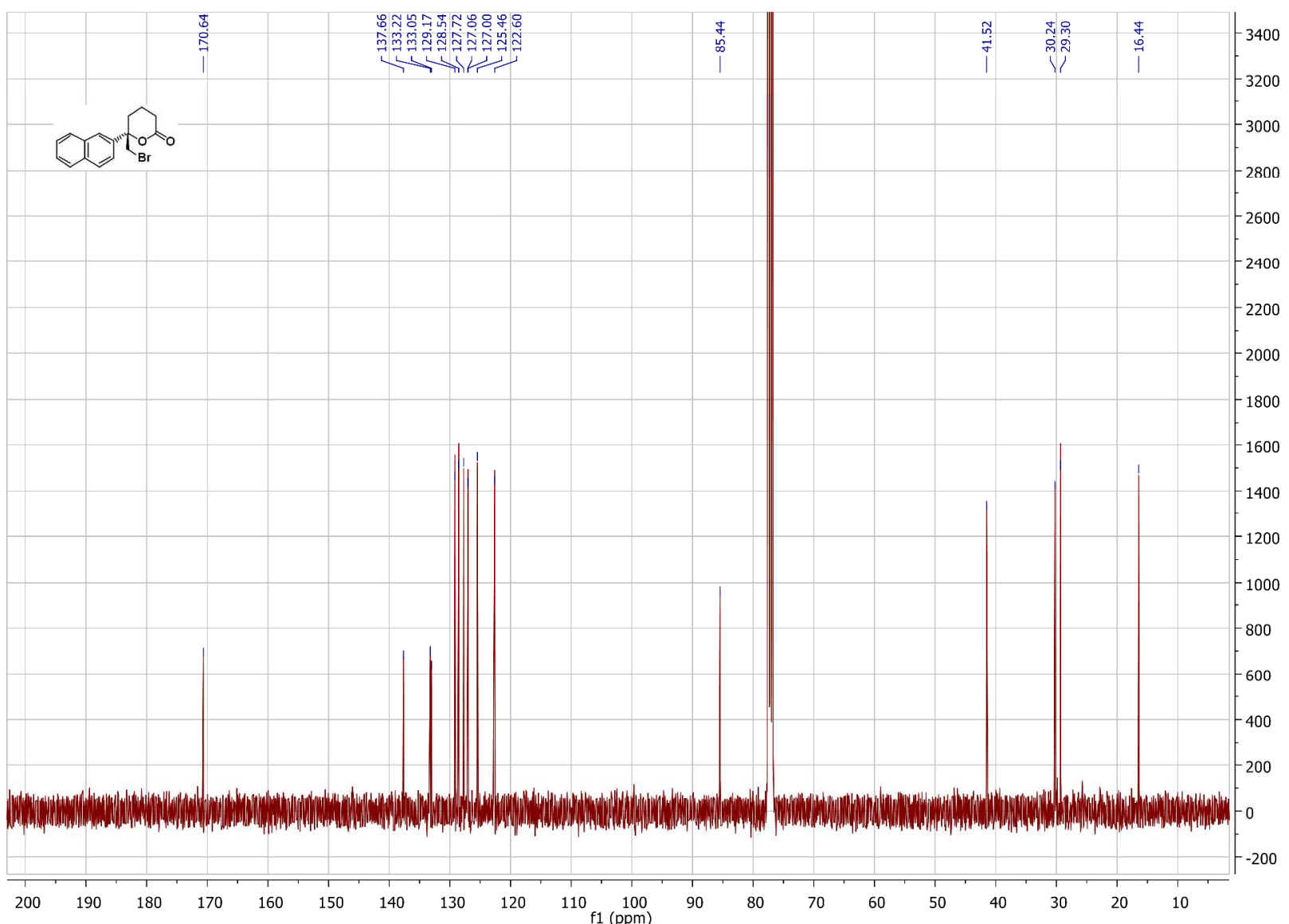

Figure S-26 ${ }^{13}$ C-NMR spectrum of compound $\mathbf{6 b}$. 


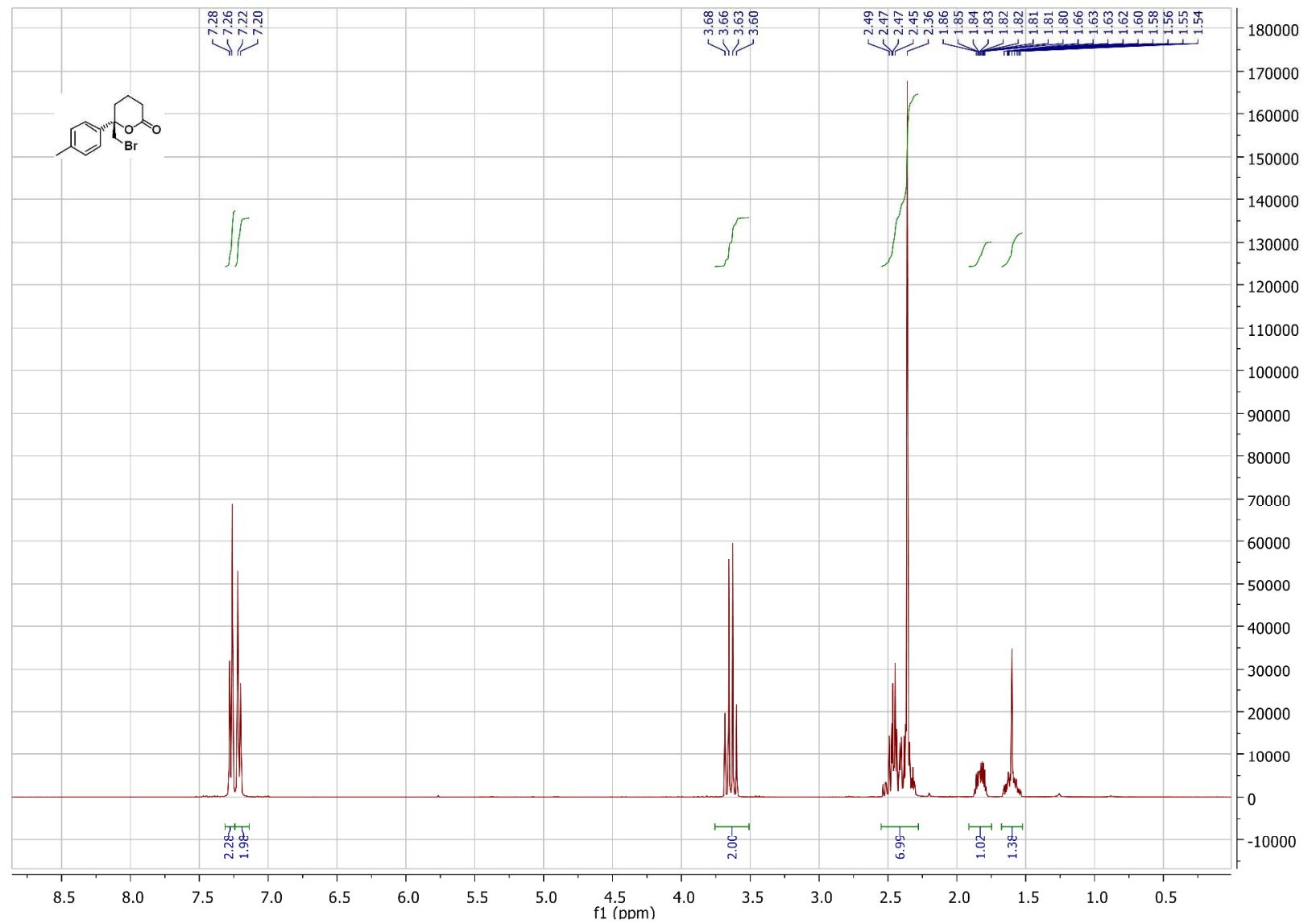

Figure S-27 ${ }^{1}$ H-NMR spectrum of compound $\mathbf{6 c}$.

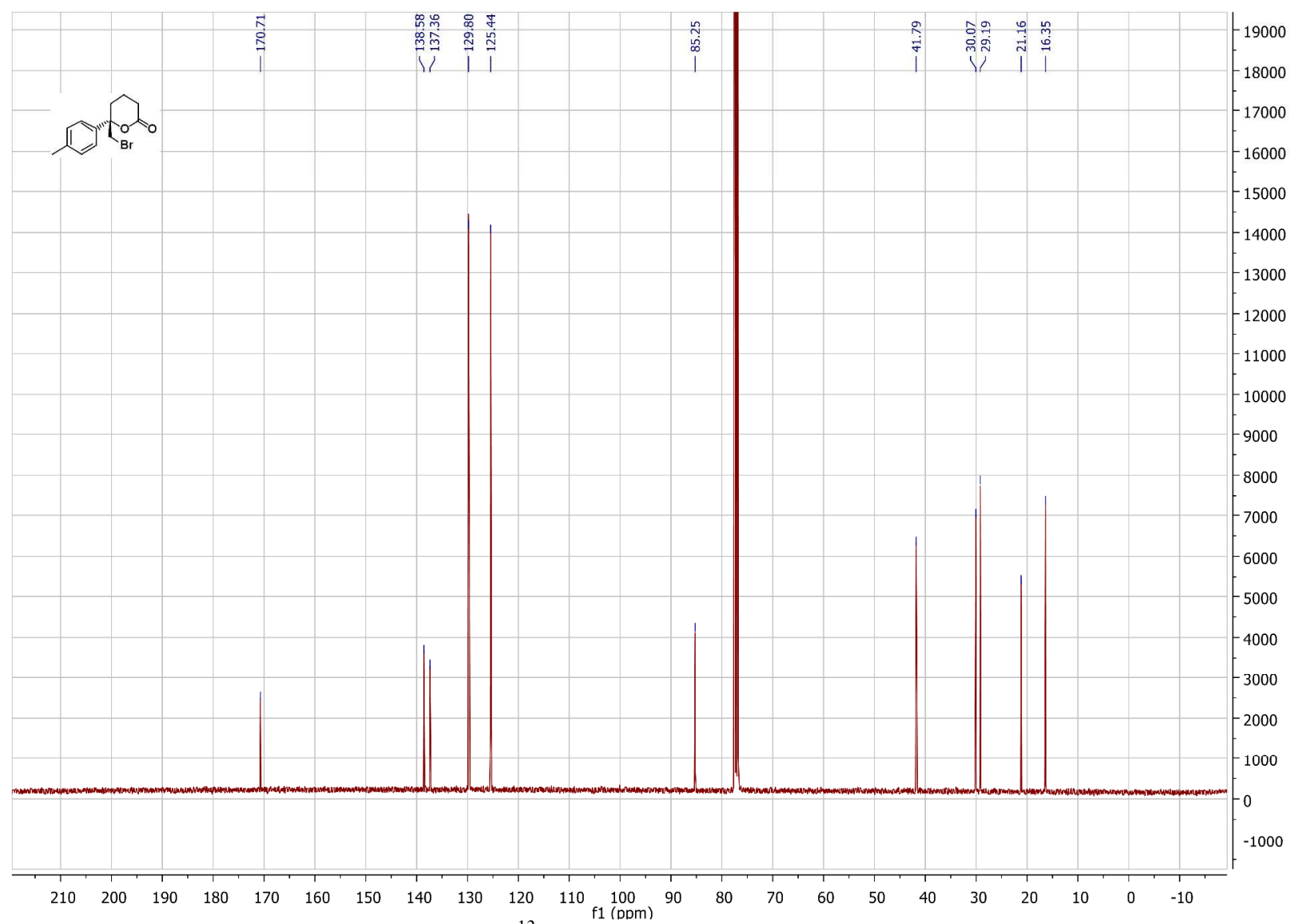

Figure S-28 ${ }^{13} \mathrm{C}-\mathrm{NMR}$ spectrum of compound $\mathbf{6 c}$. 


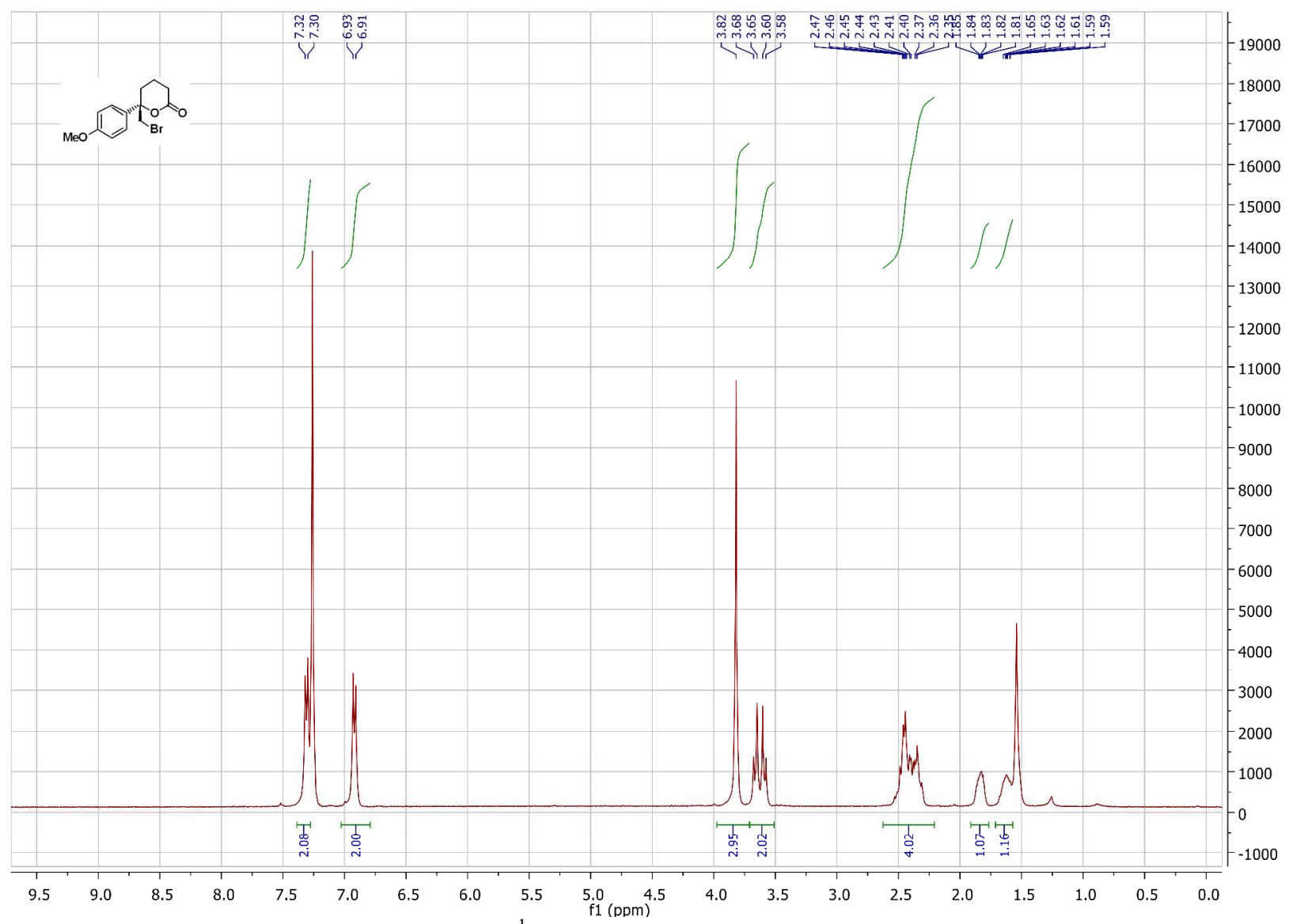

Figure S-29 ${ }^{1} \mathrm{H}-\mathrm{NMR}$ spectrum of compound $\mathbf{6 d}$.

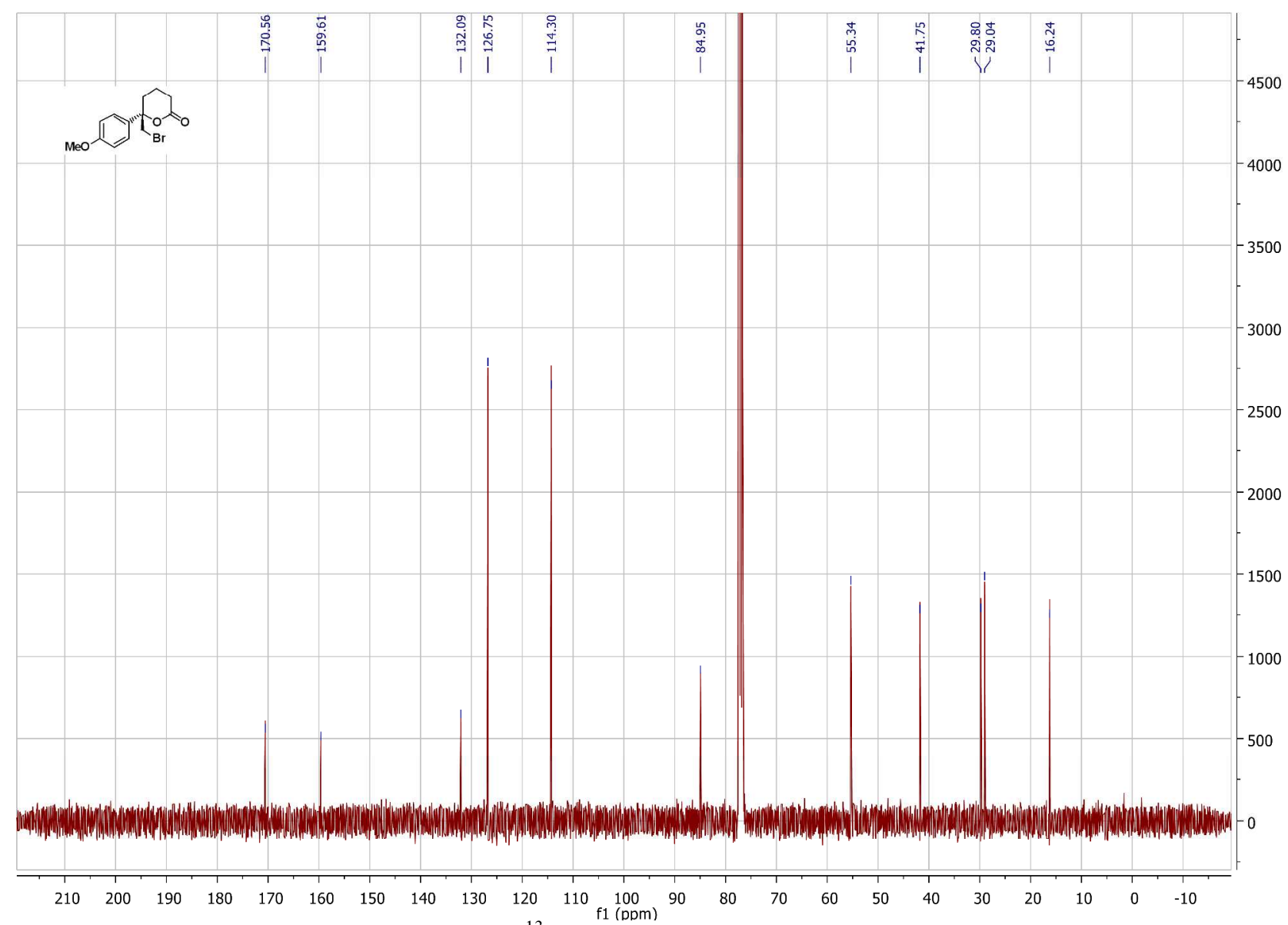

Figure S-30 ${ }^{13} \mathrm{C}-\mathrm{NMR}$ spectrum of compound $\mathbf{6 d}$. 


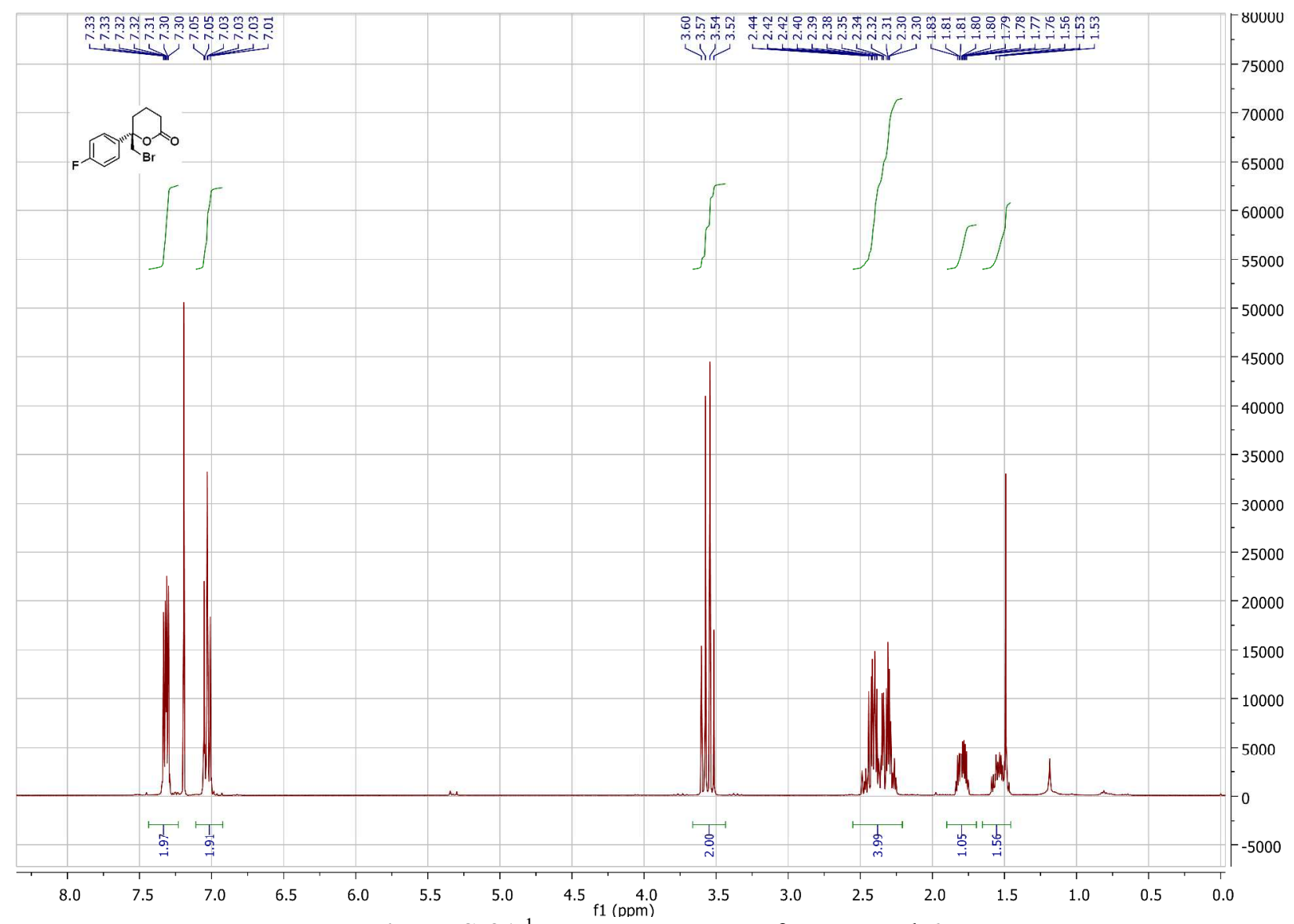

Figure S-31 ${ }^{1} \mathrm{H}-\mathrm{NMR}$ spectrum of compound $\mathbf{6 e}$.

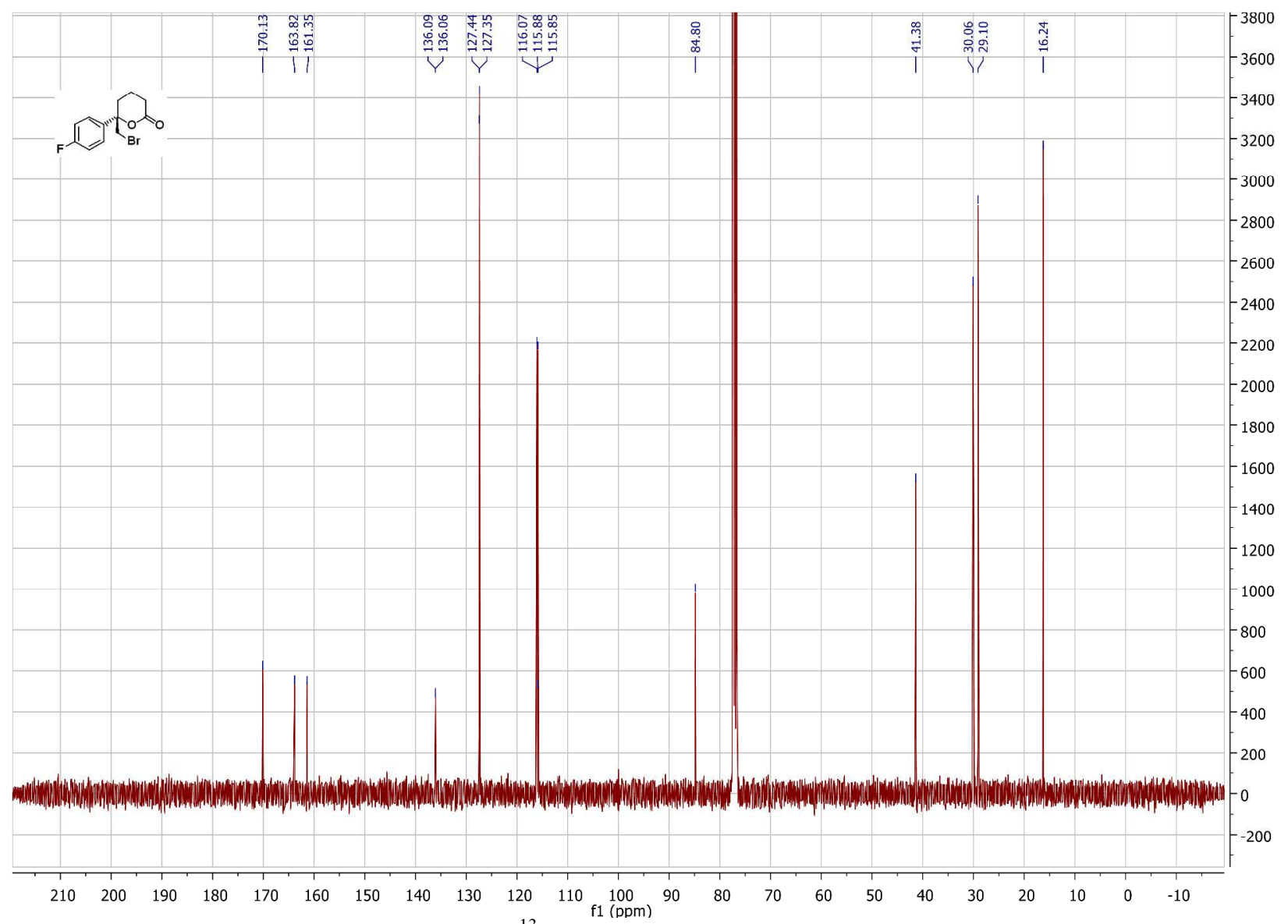

Figure S-32 ${ }^{13}$ C-NMR spectrum of compound $6 \mathbf{e}$. 


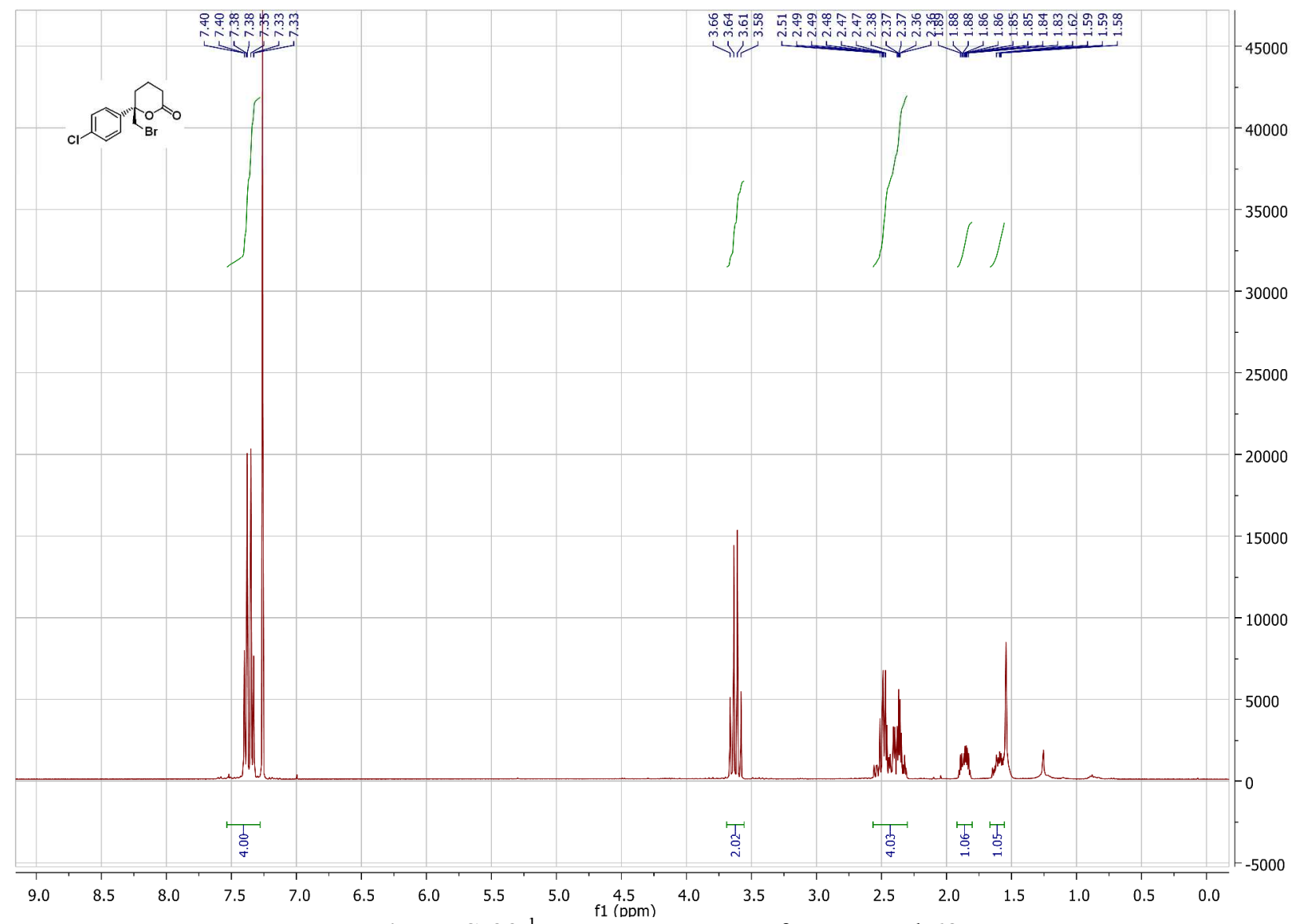

Figure S-33 ${ }^{1} \mathrm{H}-\mathrm{NMR}$ spectrum of compound $\mathbf{6 f}$.

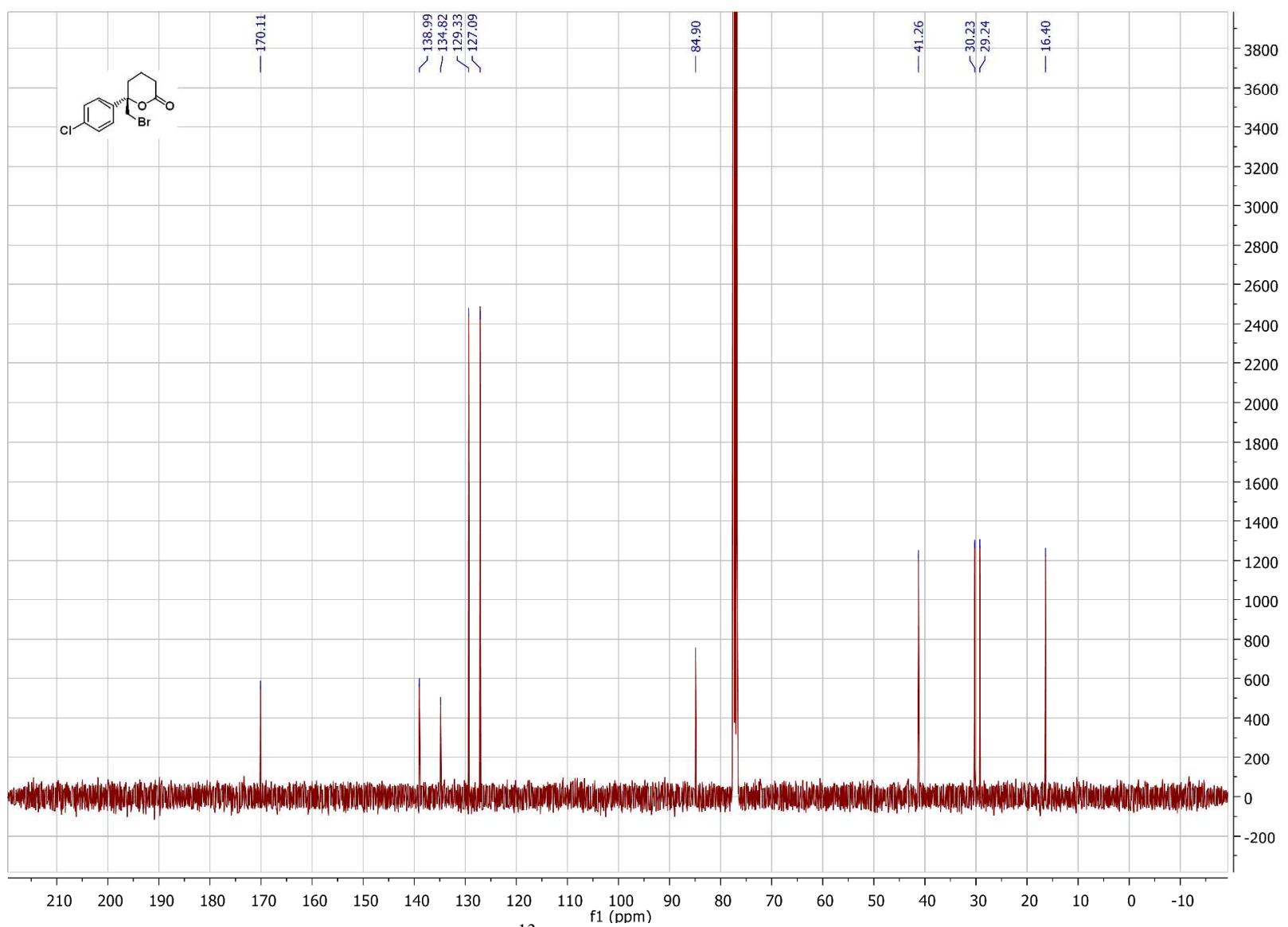

Figure S-34 ${ }^{13}$ C-NMR spectrum of compound $\mathbf{6 f}$. 


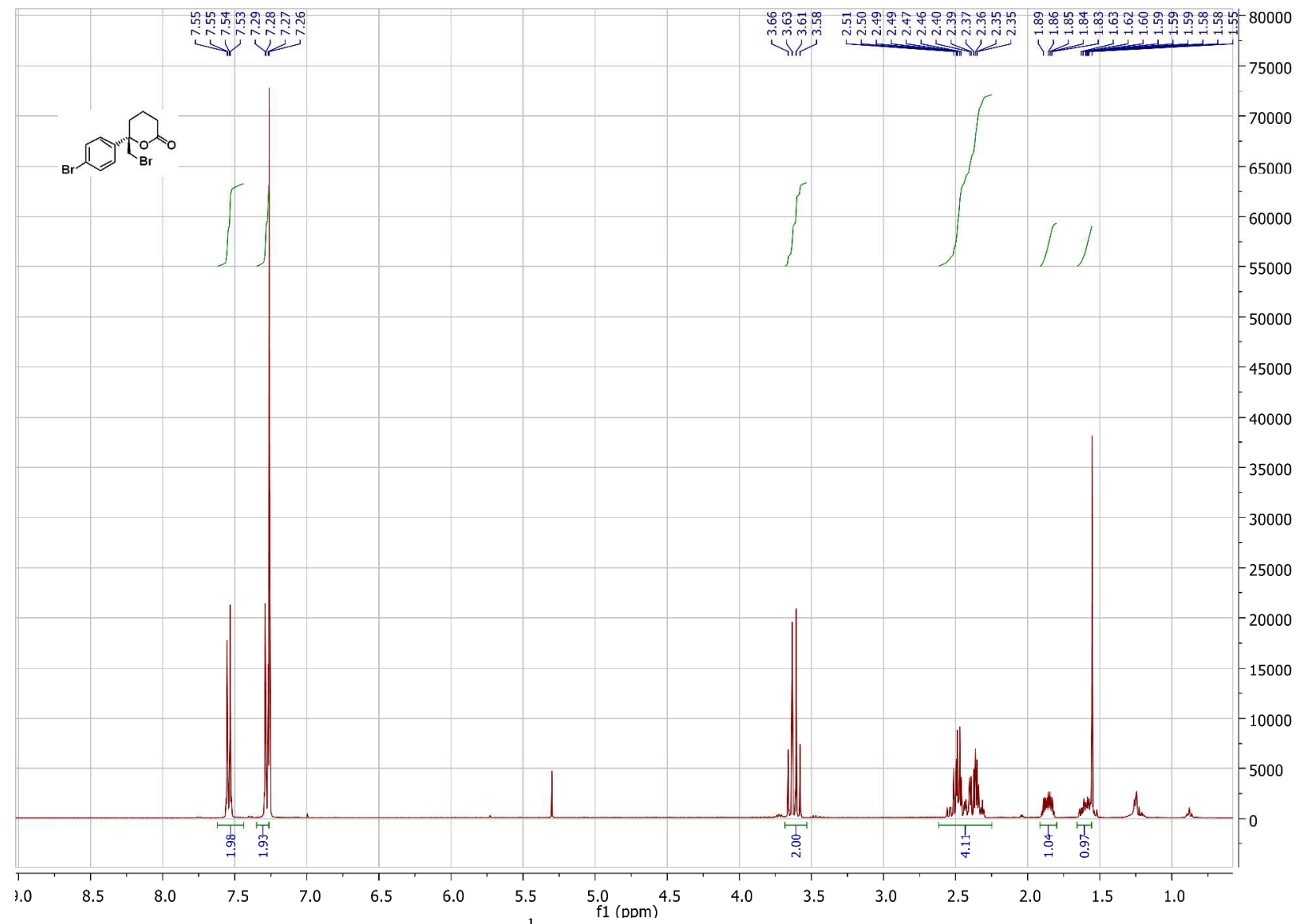

Figure S-35 ${ }^{1} \mathrm{H}-\mathrm{NMR}$ spectrum of compound $\mathbf{6 g}$.

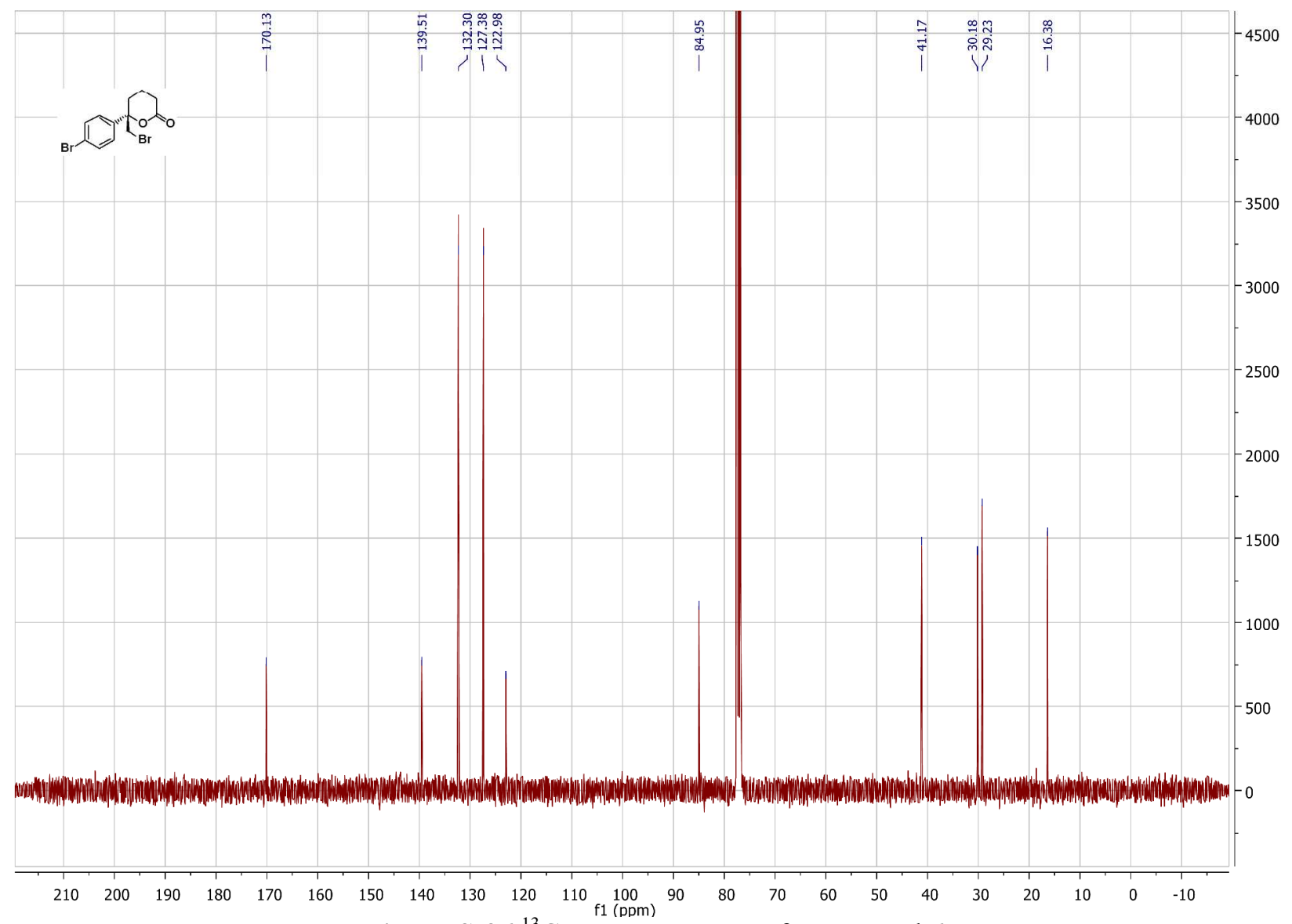

Figure S-36 ${ }^{13} \mathrm{C}-\mathrm{NMR}$ spectrum of compound $\mathbf{6 g}$. 


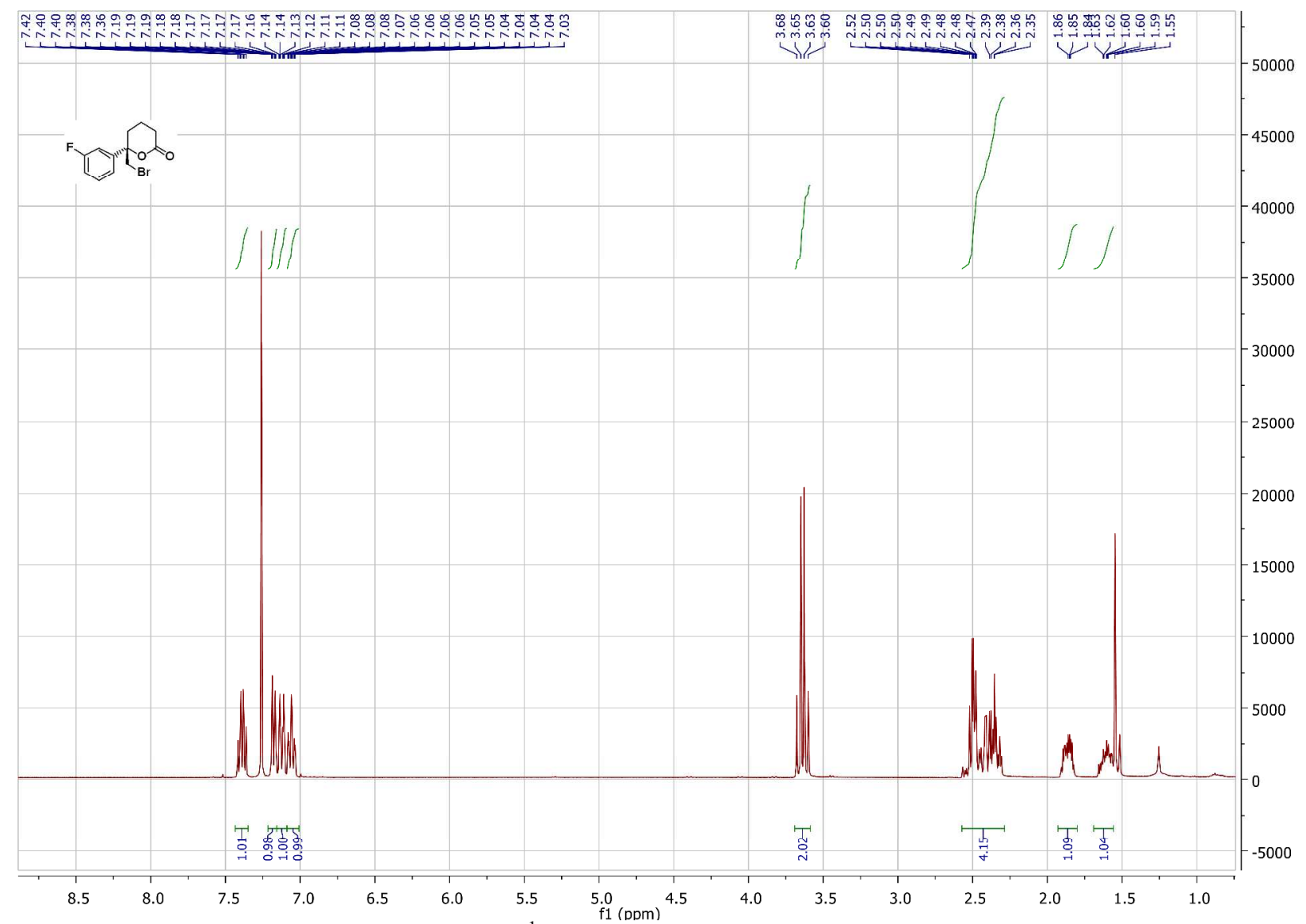

Figure S-37 ${ }^{1}$ H-NMR spectrum of compound $6 \mathbf{h}$.

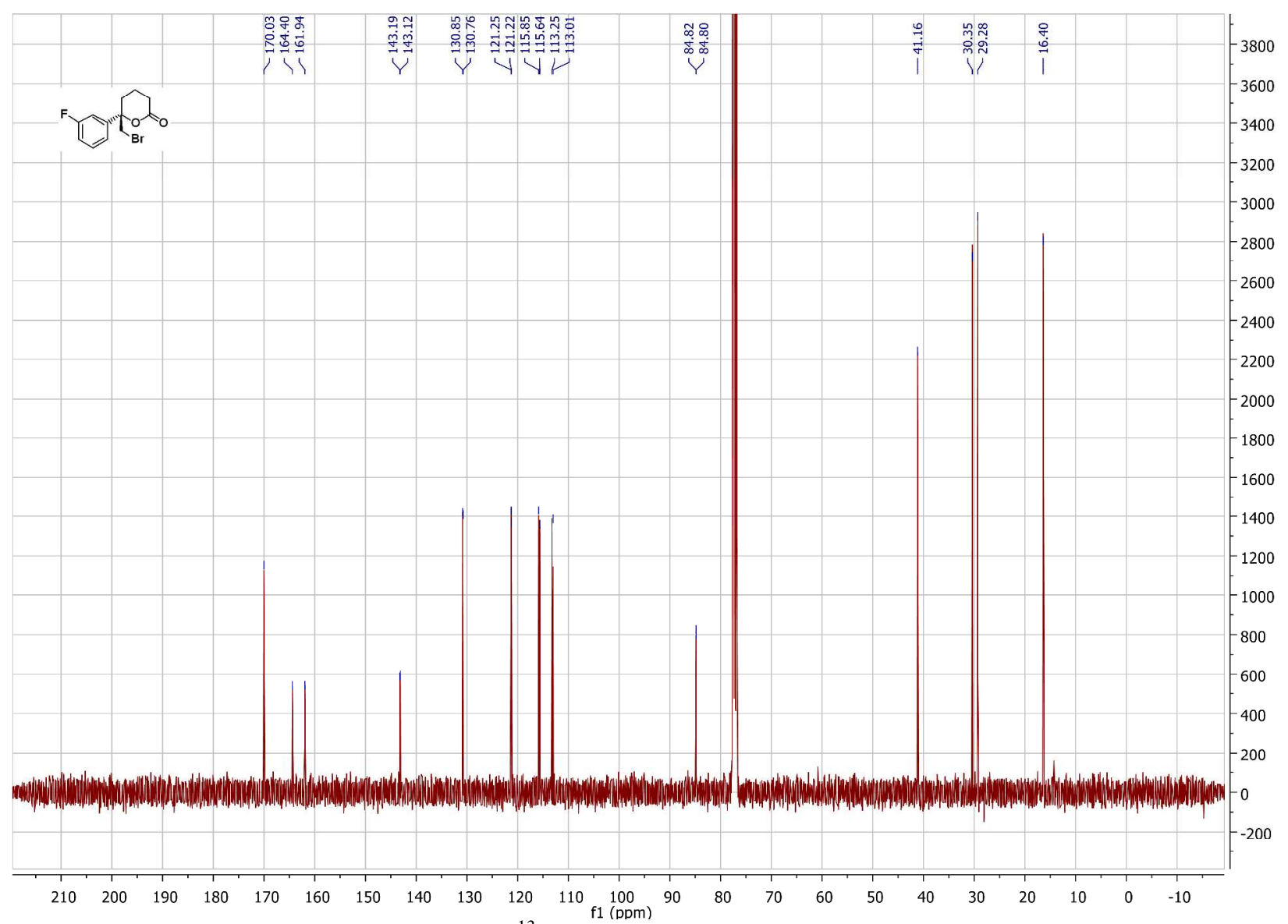

Figure S-38 ${ }^{13}$ C-NMR spectrum of compound $6 \mathbf{h}$. 


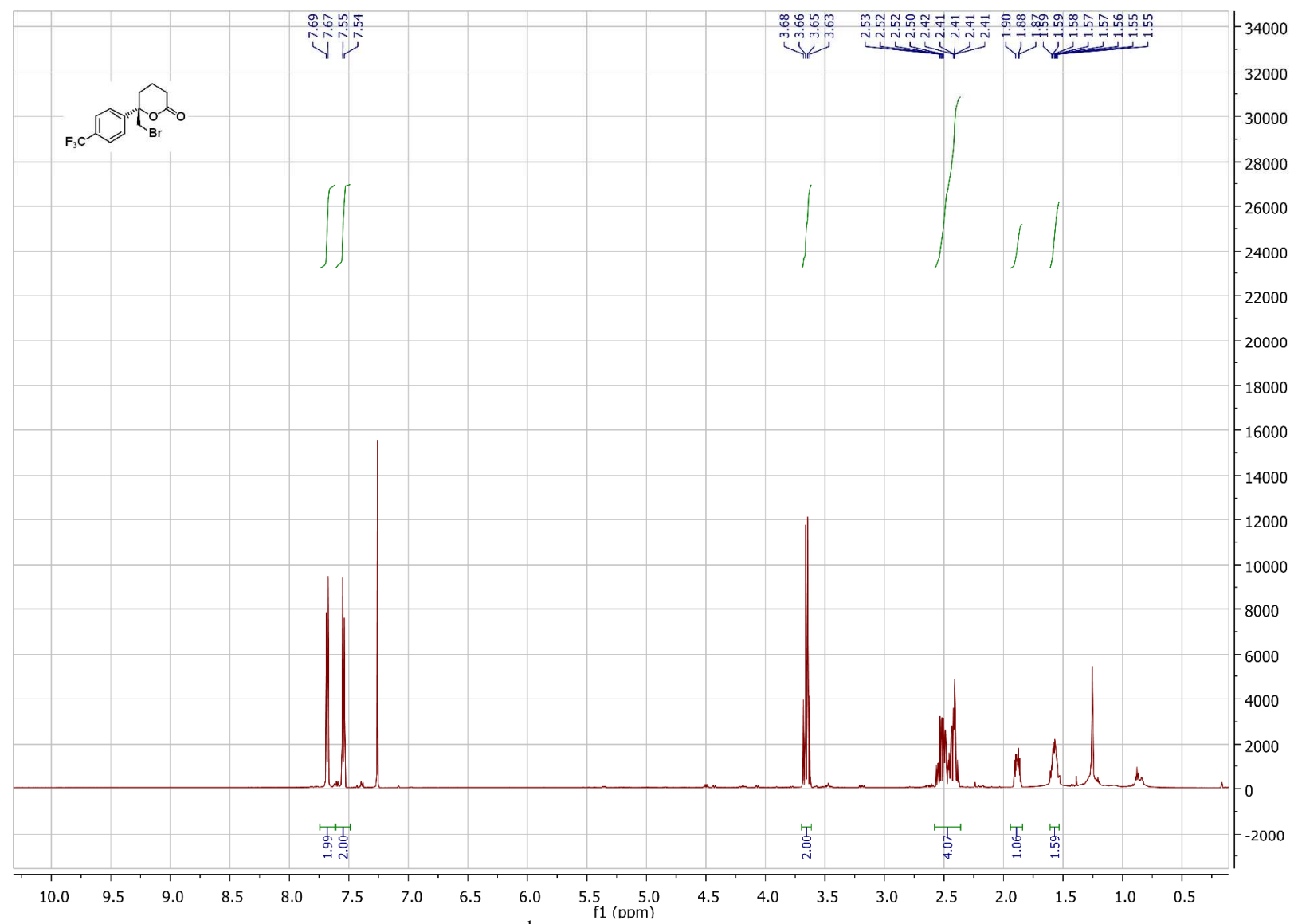

Figure S-39 ${ }^{1}$ H-NMR spectrum of compound $6 \mathbf{i}$.

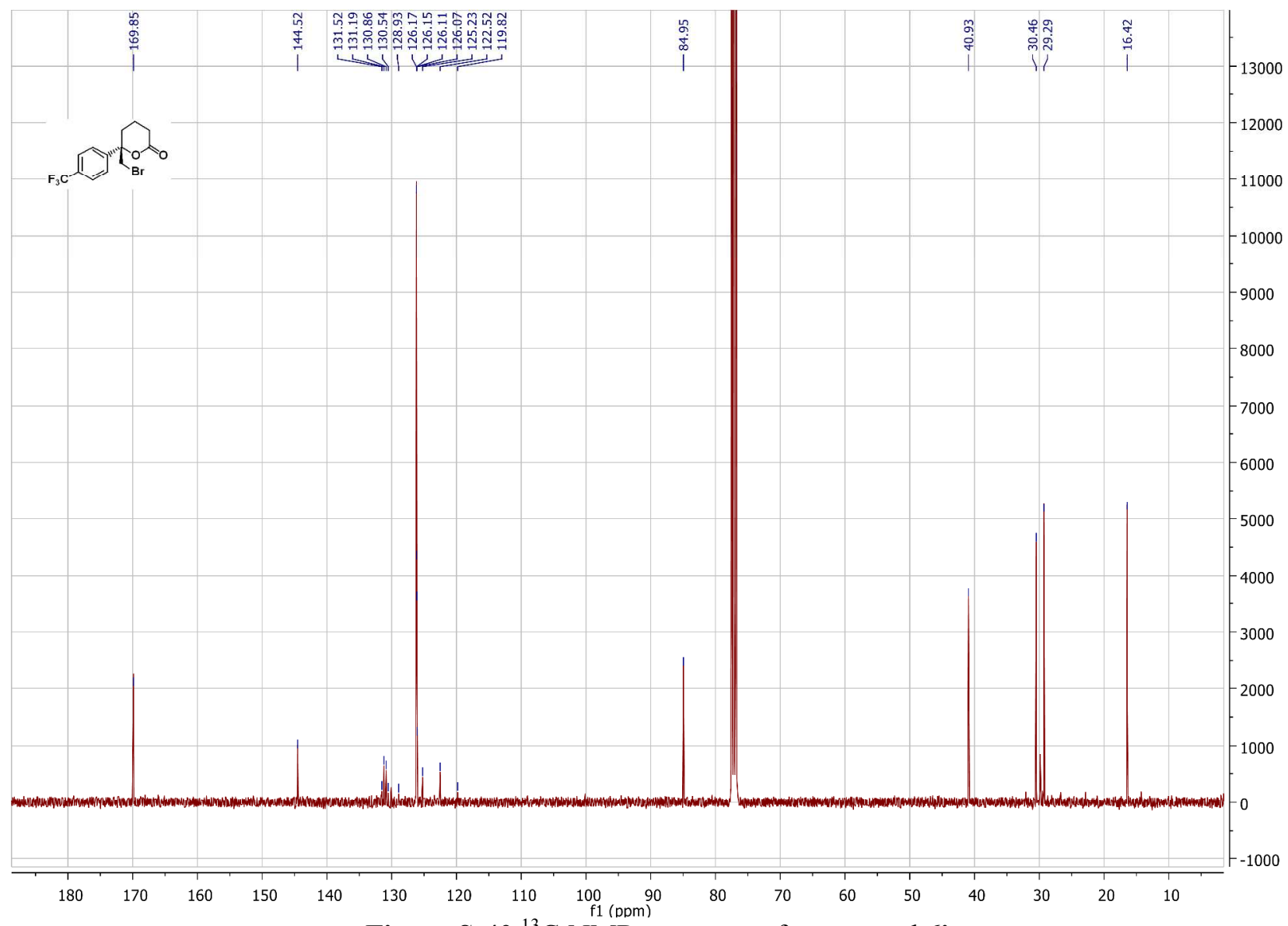

Figure S-40 ${ }^{13} \mathrm{C}-\mathrm{NMR}$ spectrum of compound $\mathbf{6 i}$. 


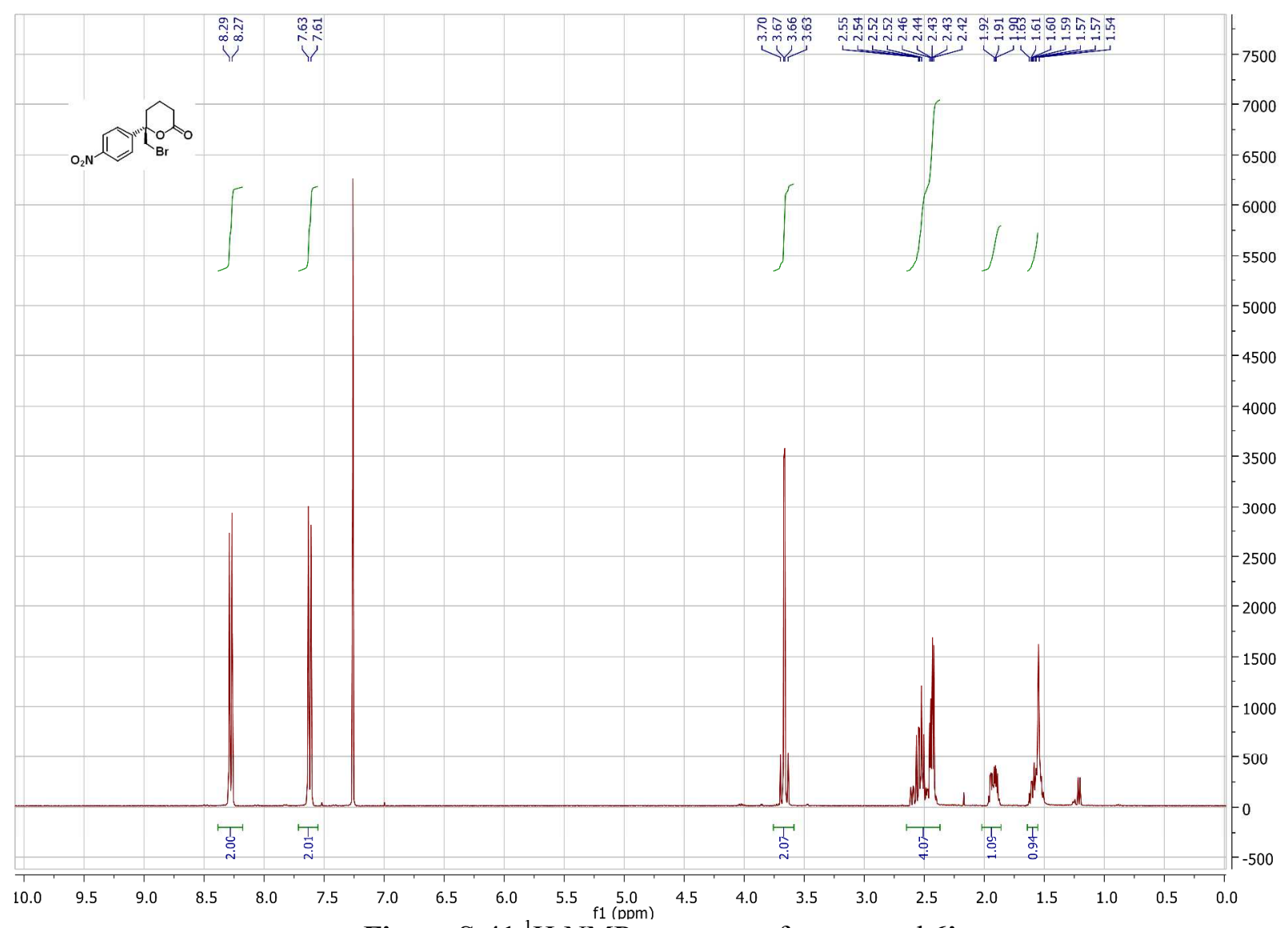

Figure S-41 ${ }^{1} \mathrm{H}-\mathrm{NMR}$ spectrum of compound $\mathbf{6 j}$.

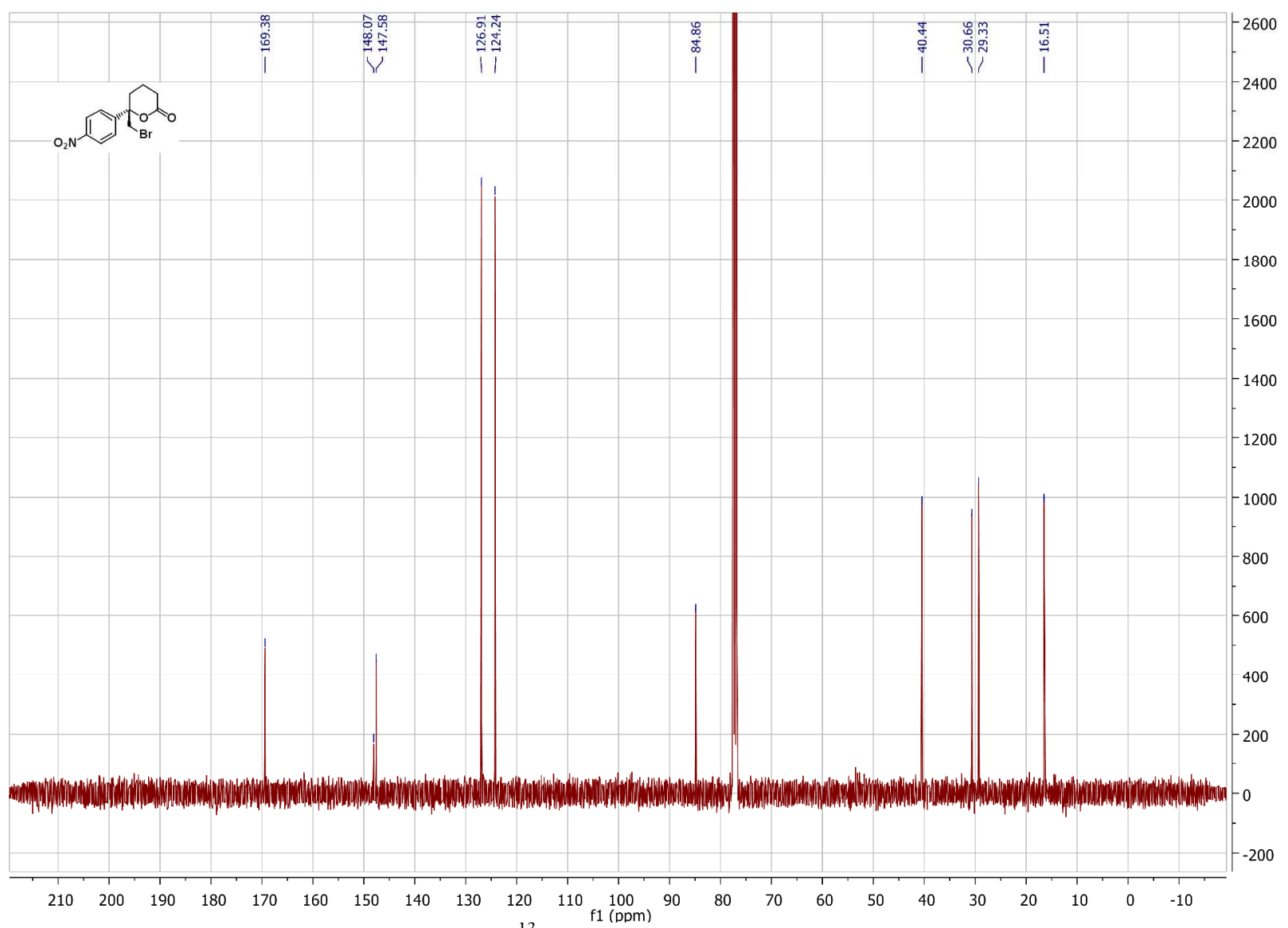

Figure S-42 ${ }^{13} \mathrm{C}-\mathrm{NMR}$ spectrum of compound $\mathbf{6 j}$. 


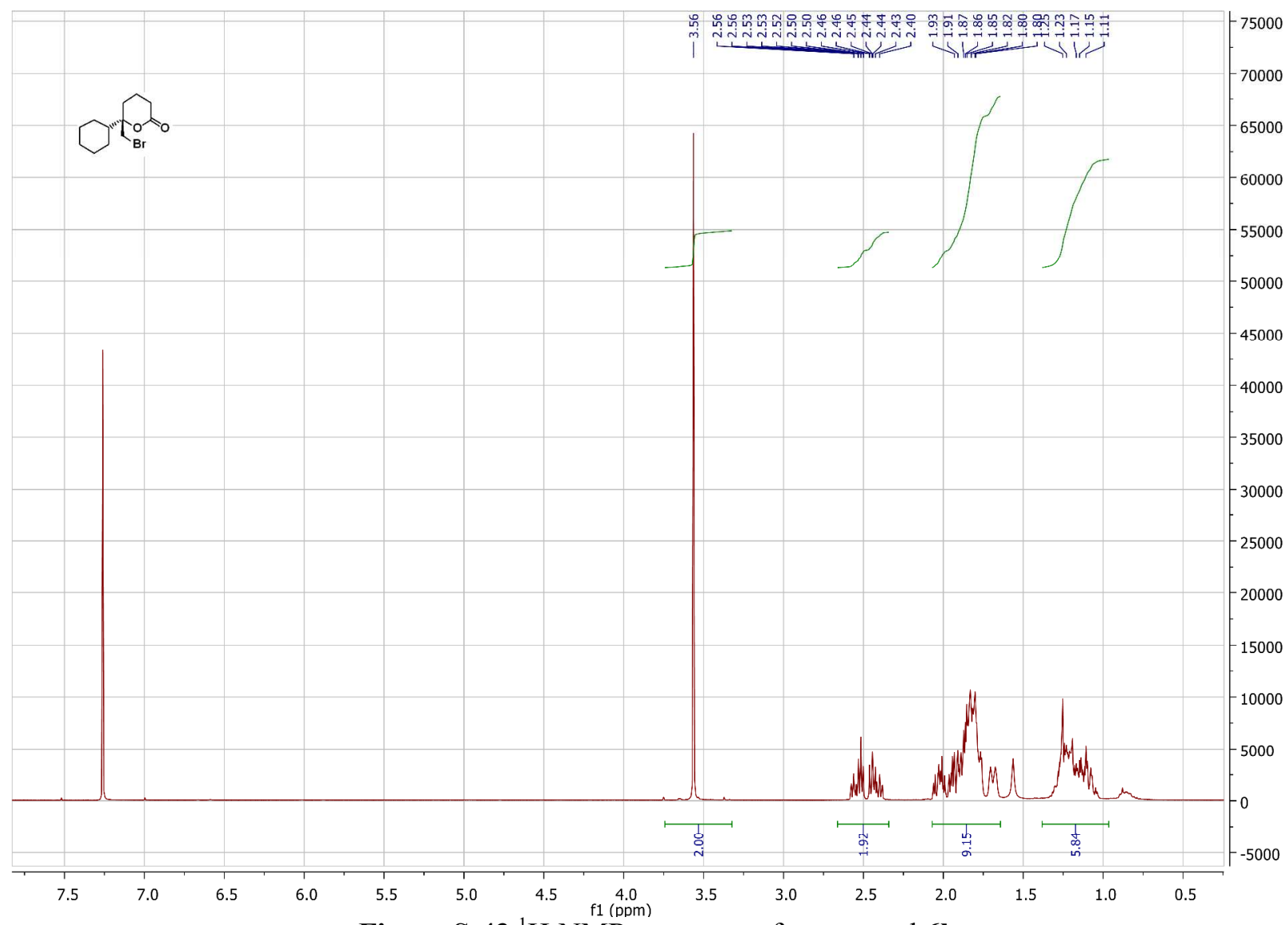

Figure S-43 ${ }^{1}$ H-NMR spectrum of compound $\mathbf{6 k}$.

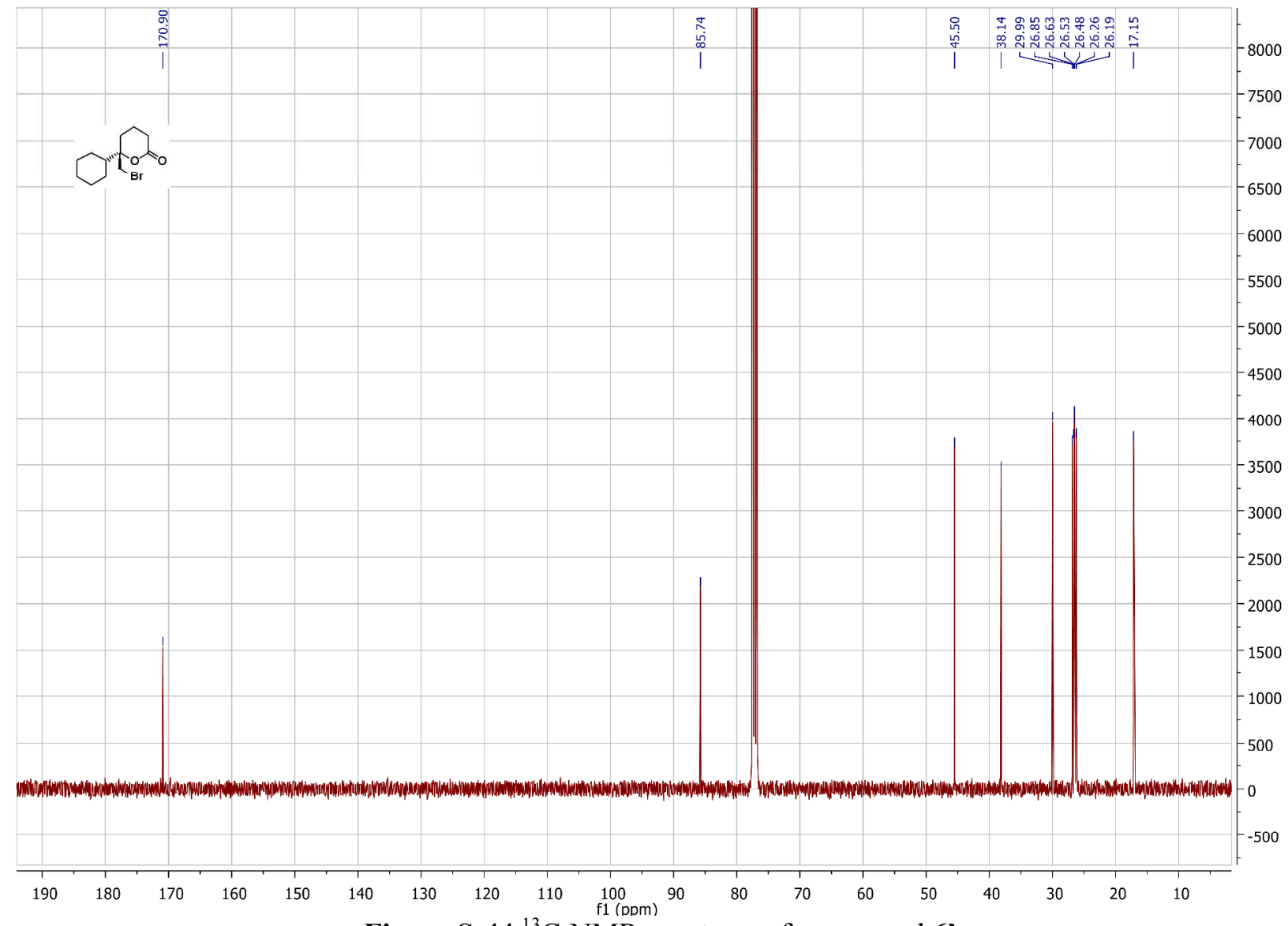

Figure S-44 ${ }^{13} \mathrm{C}-\mathrm{NMR}$ spectrum of compound $\mathbf{6 k}$. 


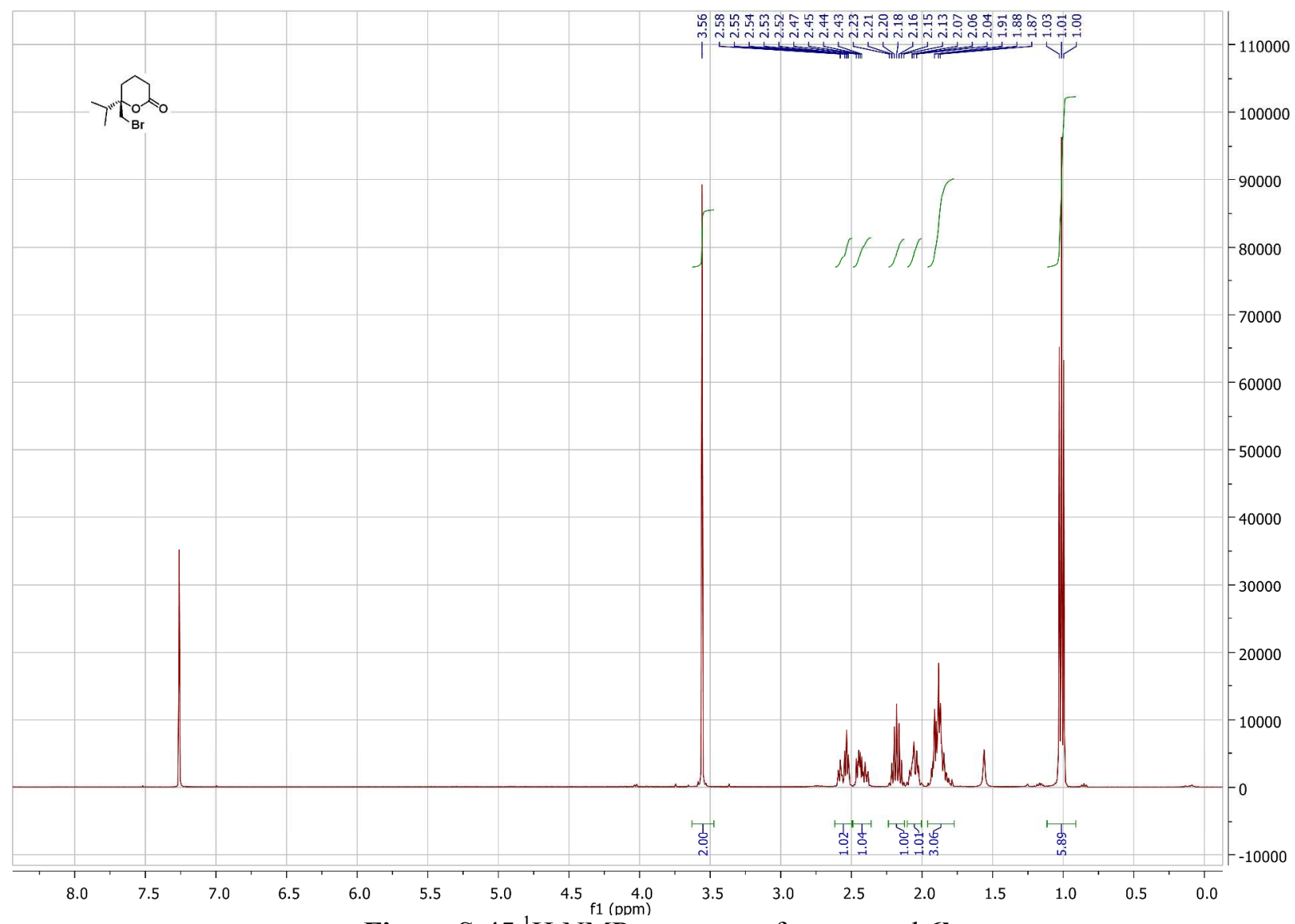

Figure S-45 ${ }^{1} \mathrm{H}-\mathrm{NMR}$ spectrum of compound $6 \mathbf{6}$.

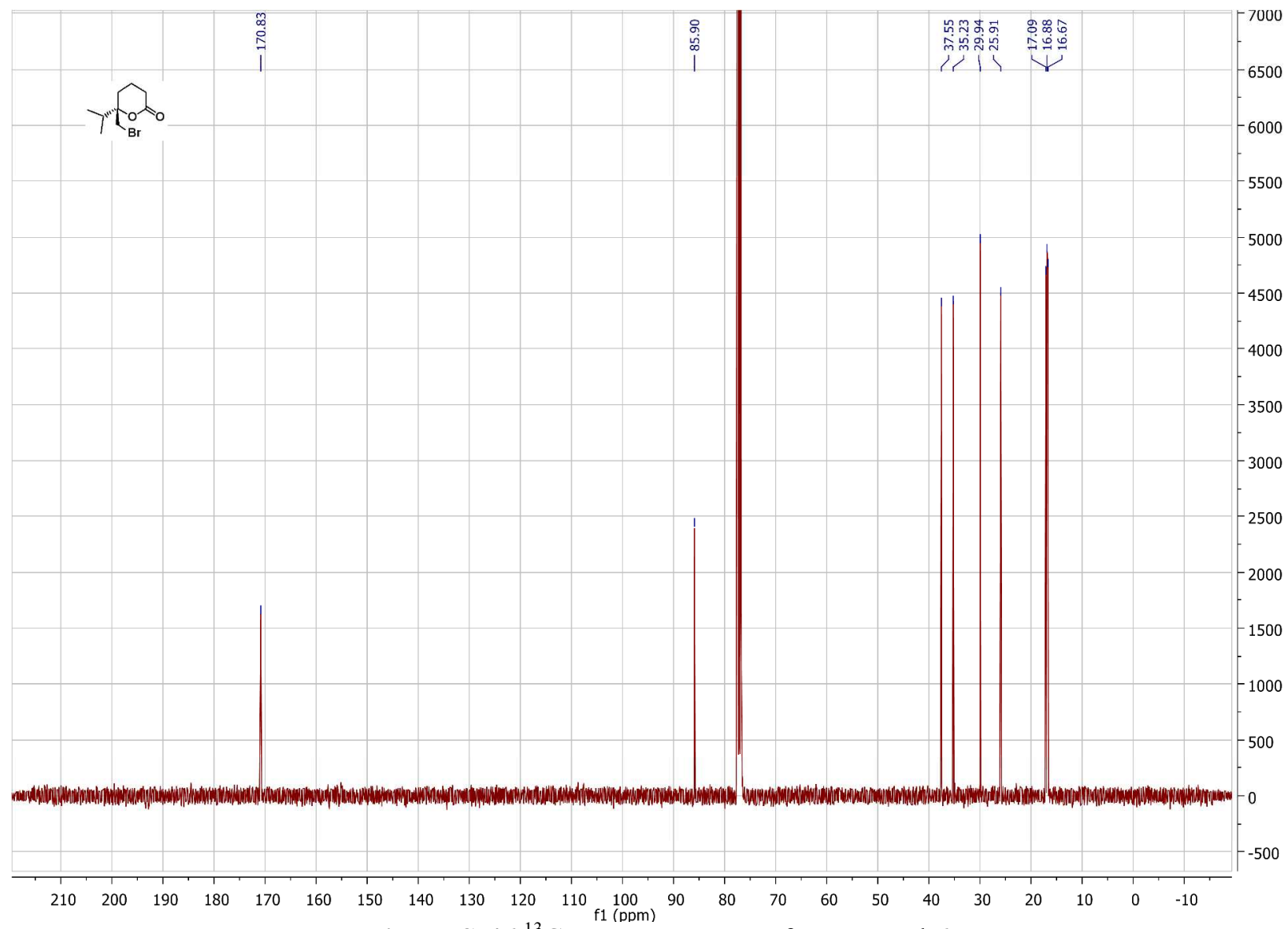

Figure S-46 ${ }^{13} \mathrm{C}-\mathrm{NMR}$ spectrum of compound $6 \mathbf{6}$. 


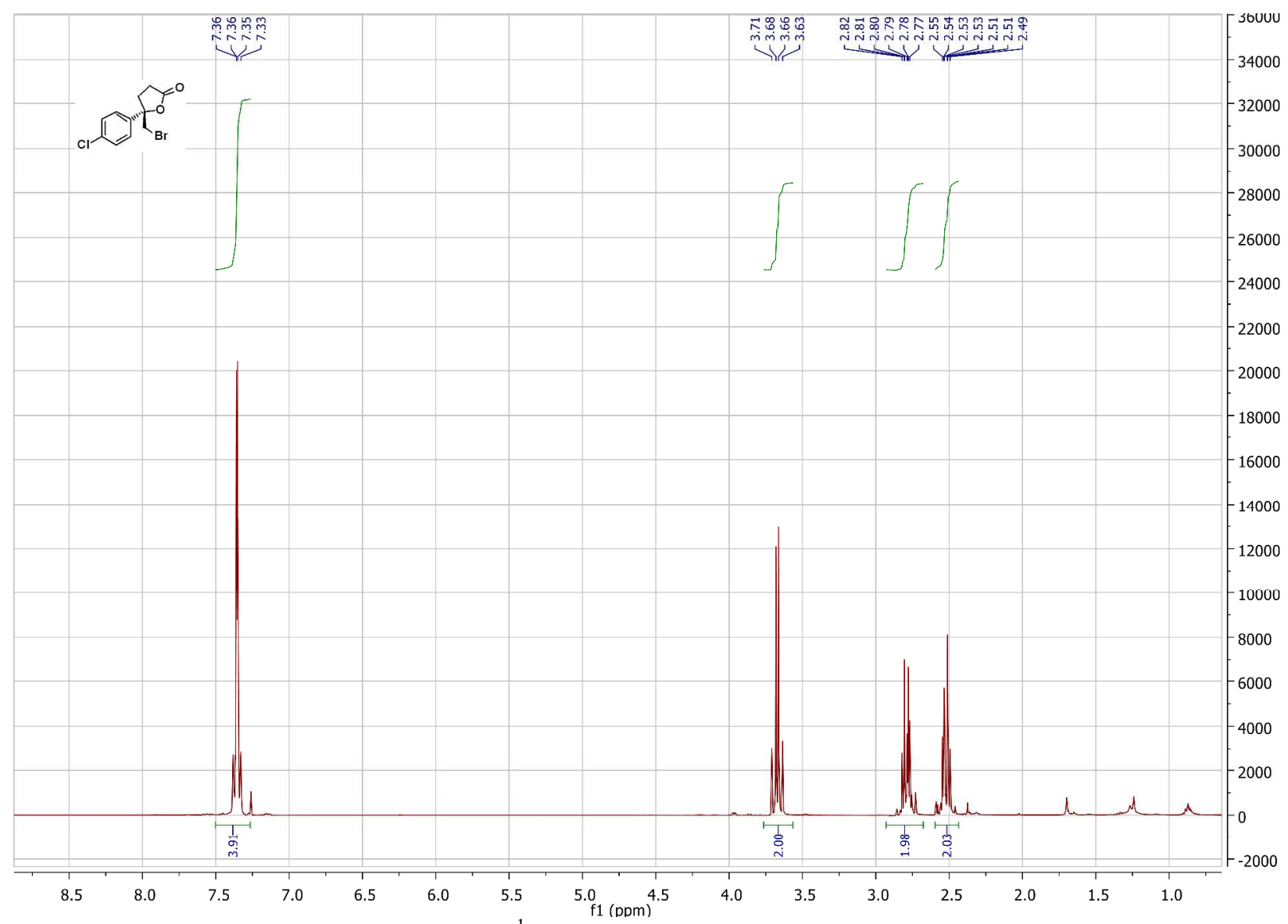

Figure S-47 ${ }^{1} \mathrm{H}-\mathrm{NMR}$ spectrum of compound $\mathbf{6 m}$.

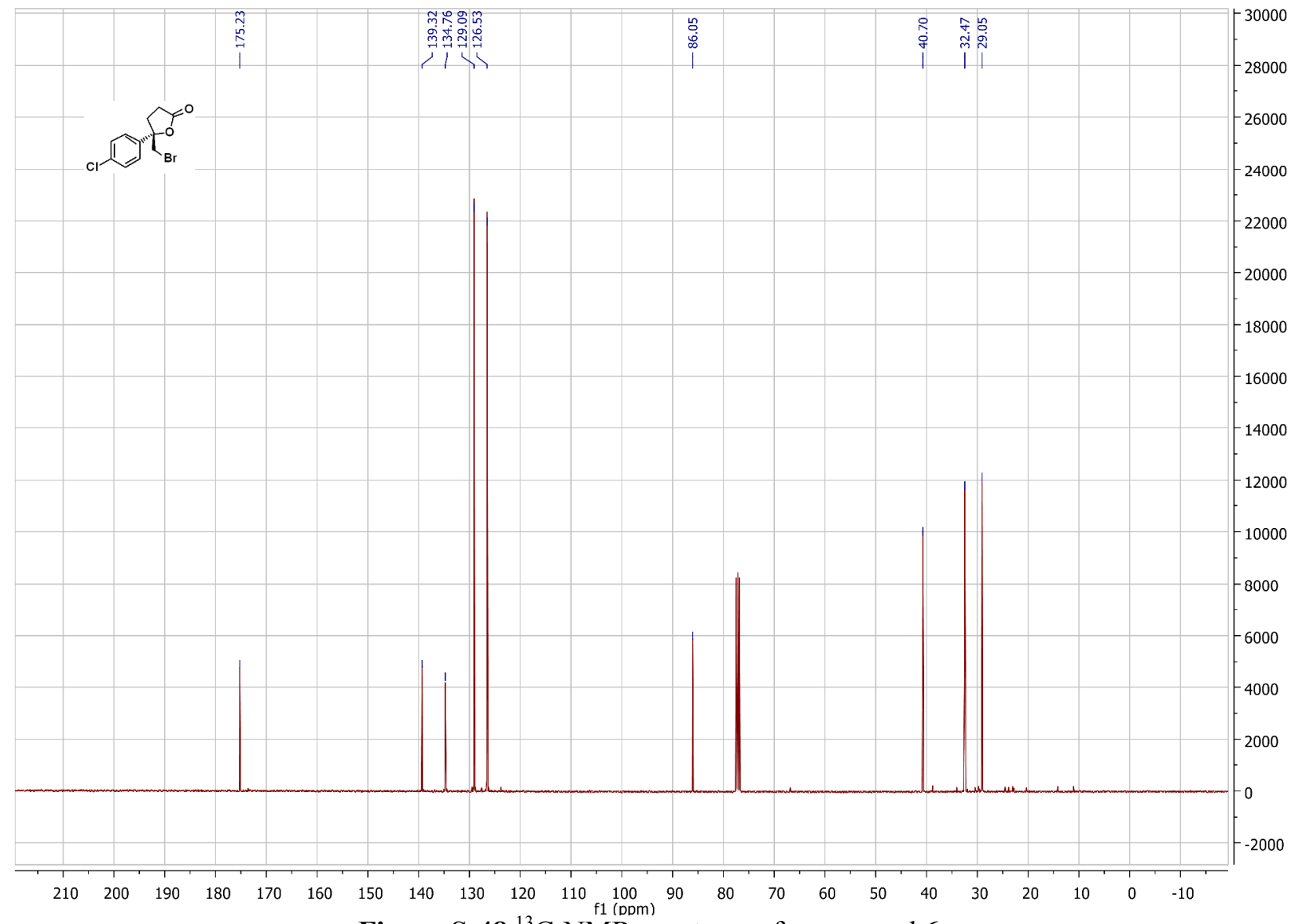

Figure S-48 ${ }^{13}$ C-NMR spectrum of compound $6 \mathbf{m}$. 


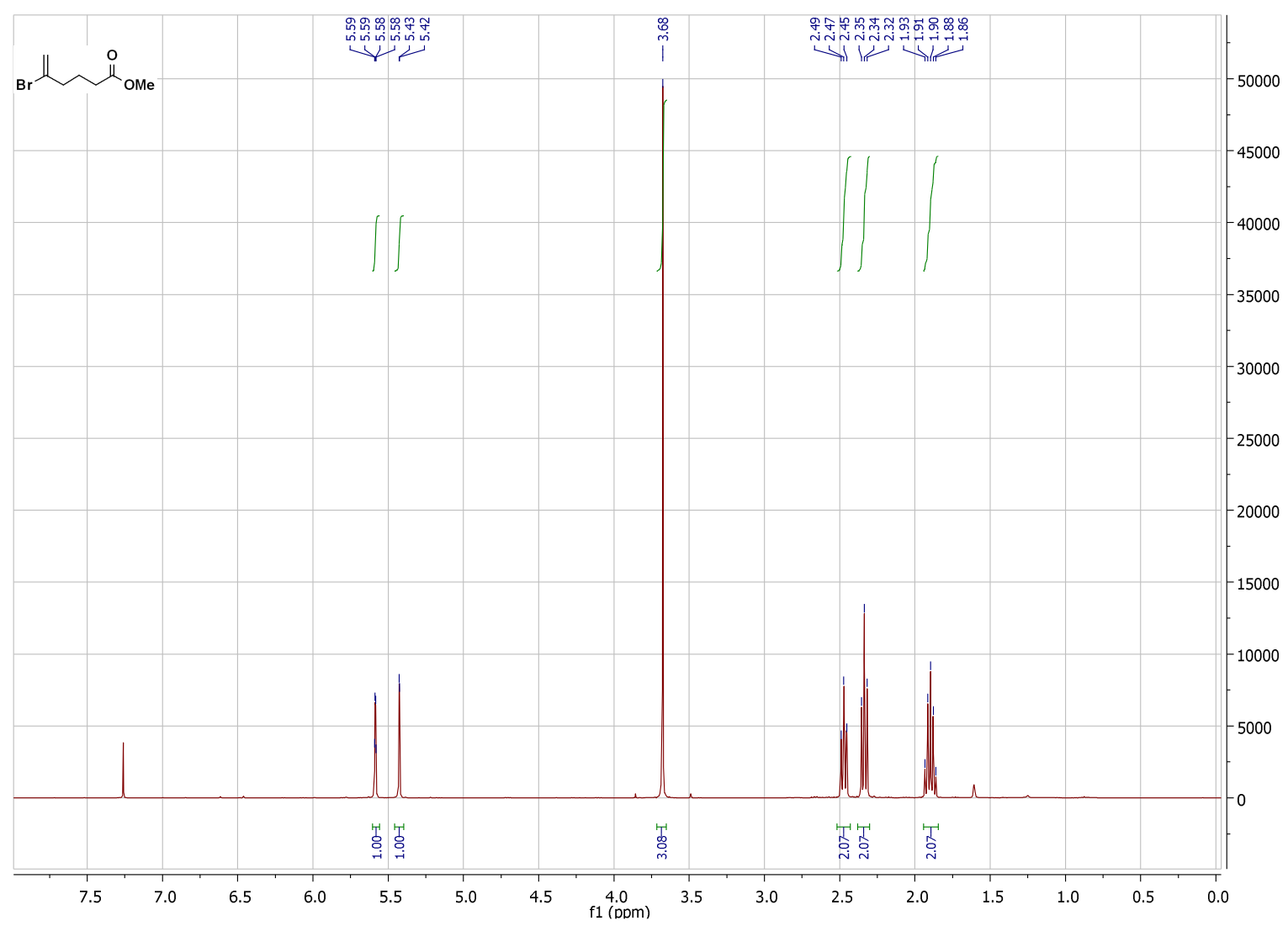

Figure S-49 ${ }^{1} \mathrm{H}-\mathrm{NMR}$ spectrum of compound $\mathbf{1 0}$.

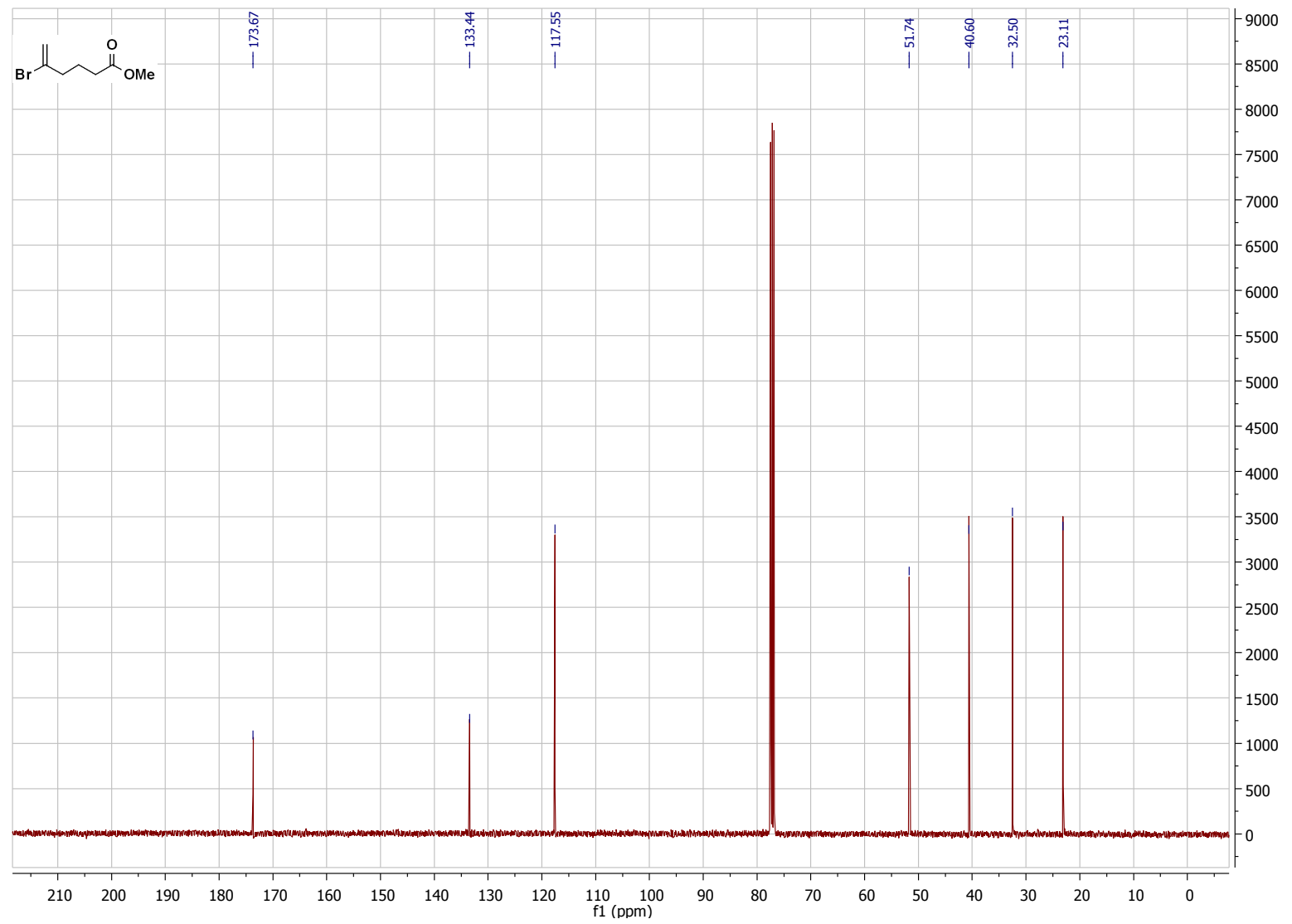

Figure S-50 ${ }^{13} \mathrm{C}-\mathrm{NMR}$ spectrum of compound $\mathbf{1 0}$. 


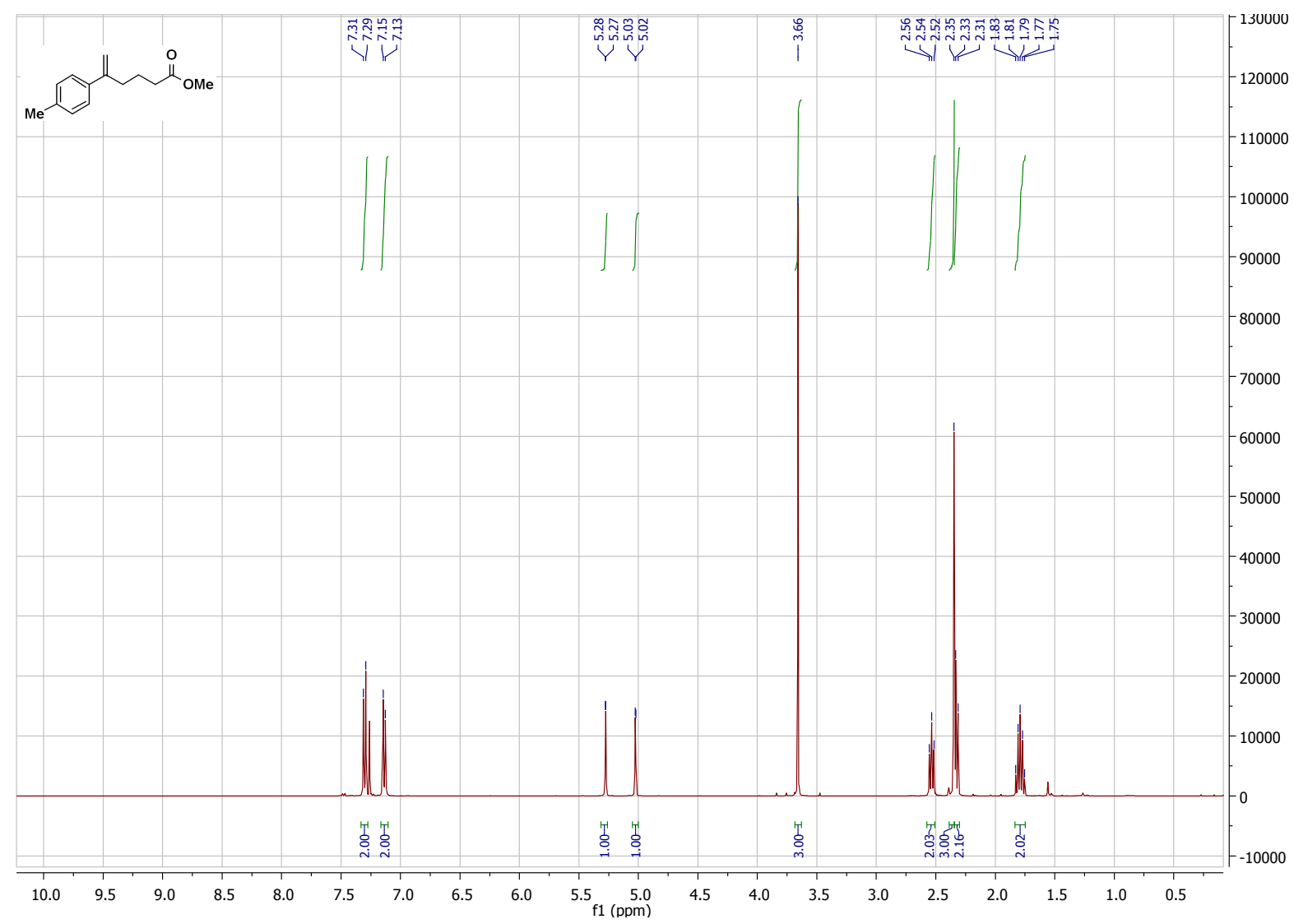

Figure S-51 ${ }^{1} \mathrm{H}-\mathrm{NMR}$ spectrum of compound 11c.

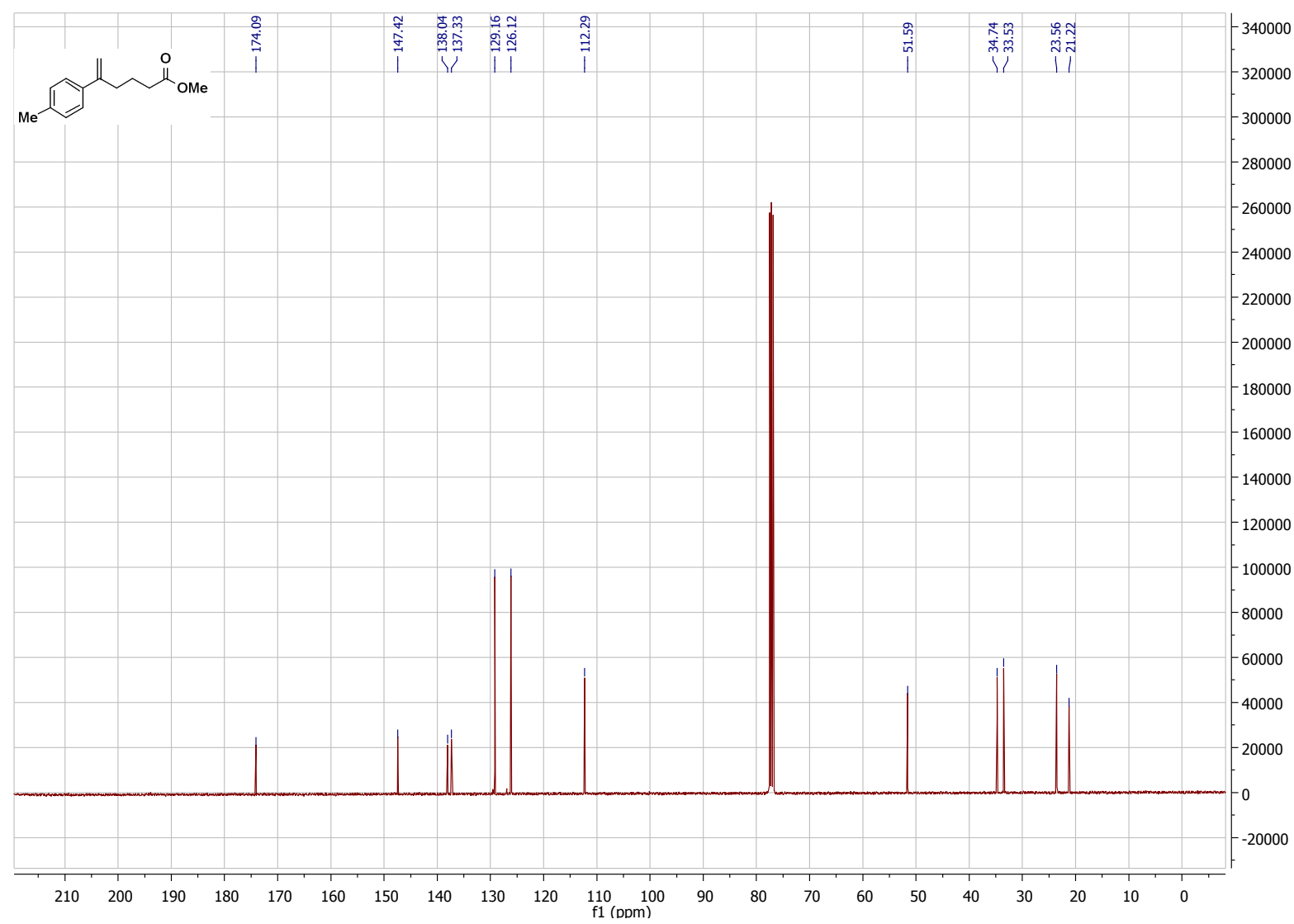

Figure S-52 ${ }^{13} \mathrm{C}-\mathrm{NMR}$ spectrum of compound 11c. 


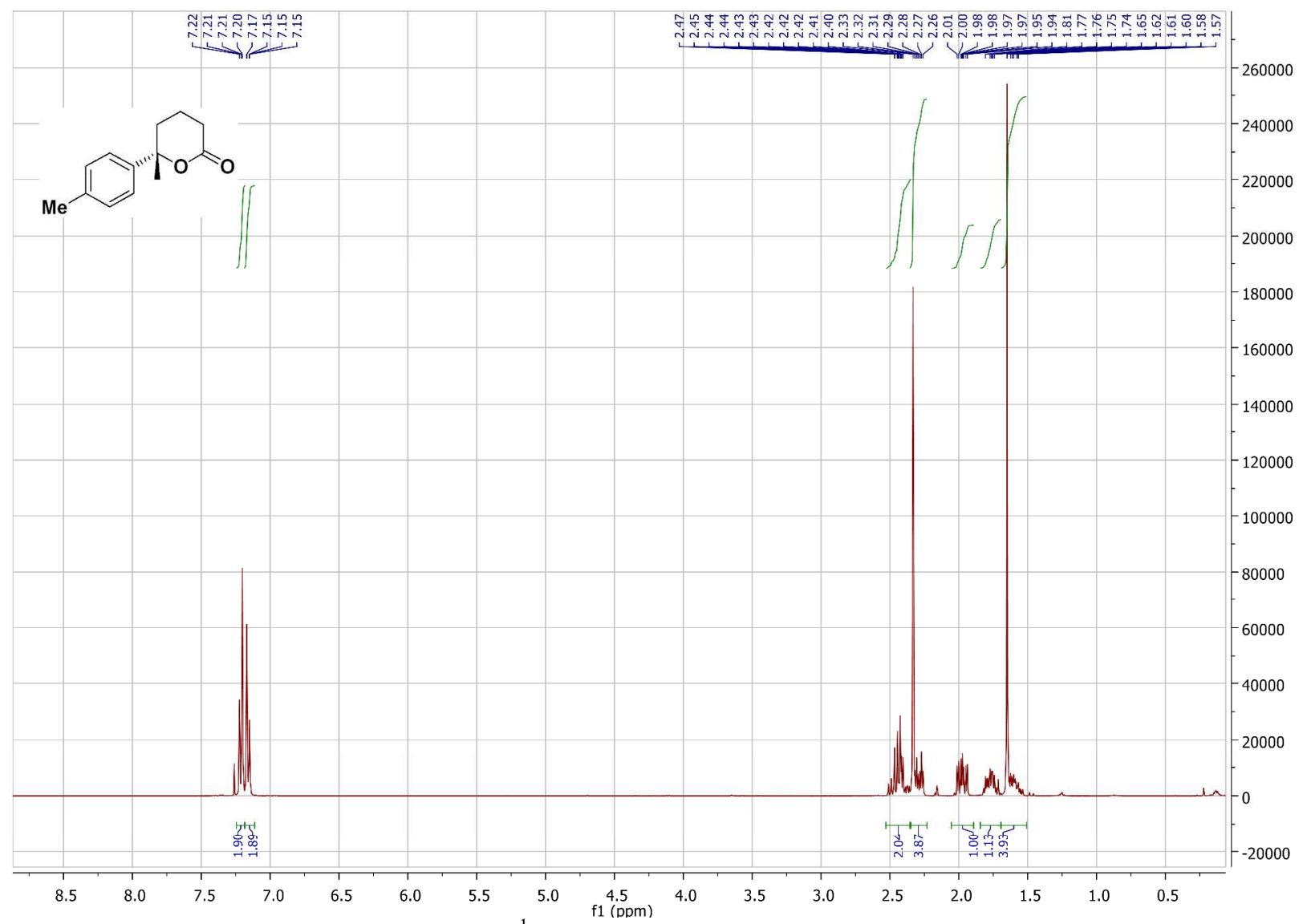

Figure S-53 ${ }^{1}$ H-NMR spectrum of compound 12.

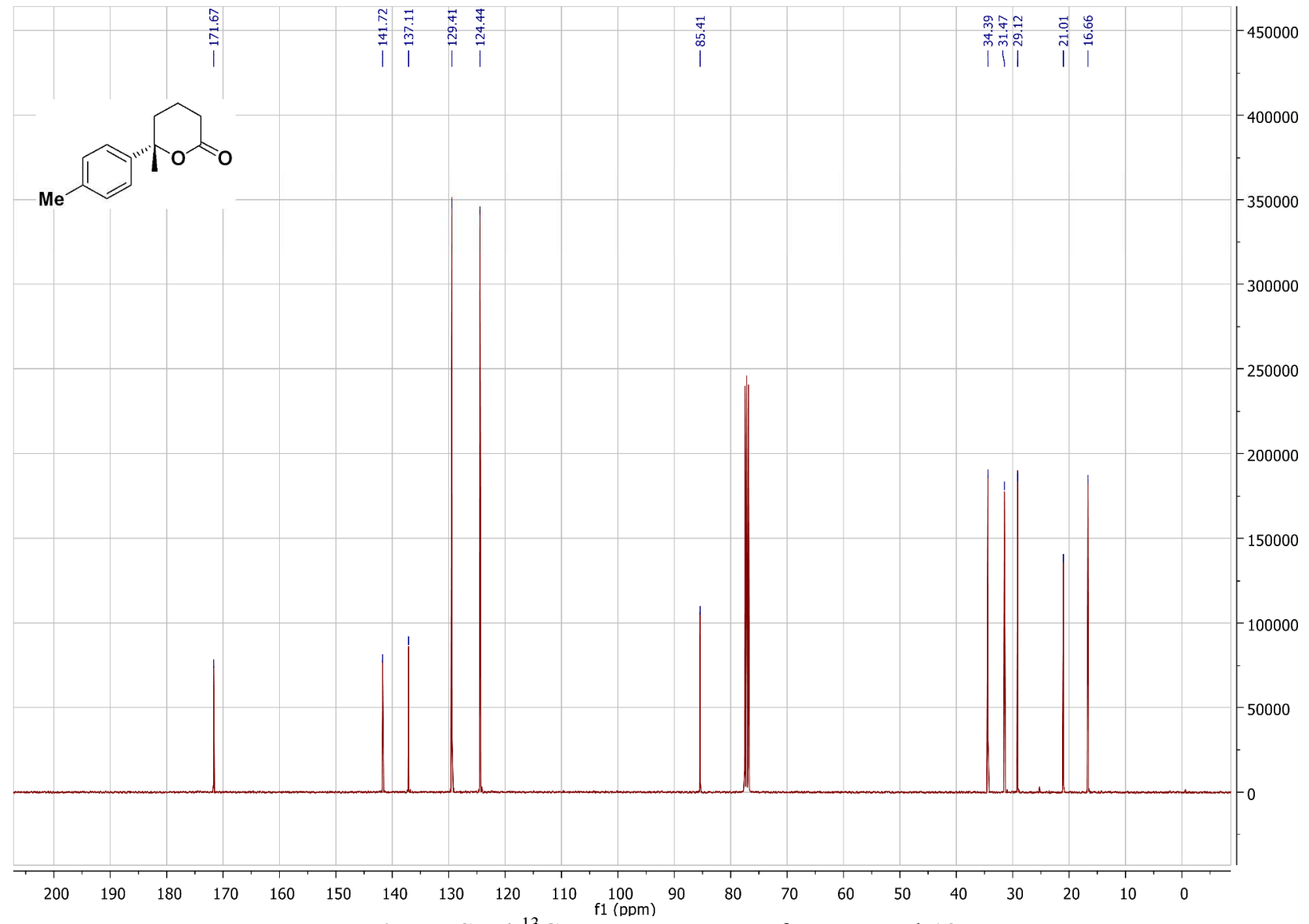

Figure S-54 ${ }^{13} \mathrm{C}-\mathrm{NMR}$ spectrum of compound $\mathbf{1 2}$. 


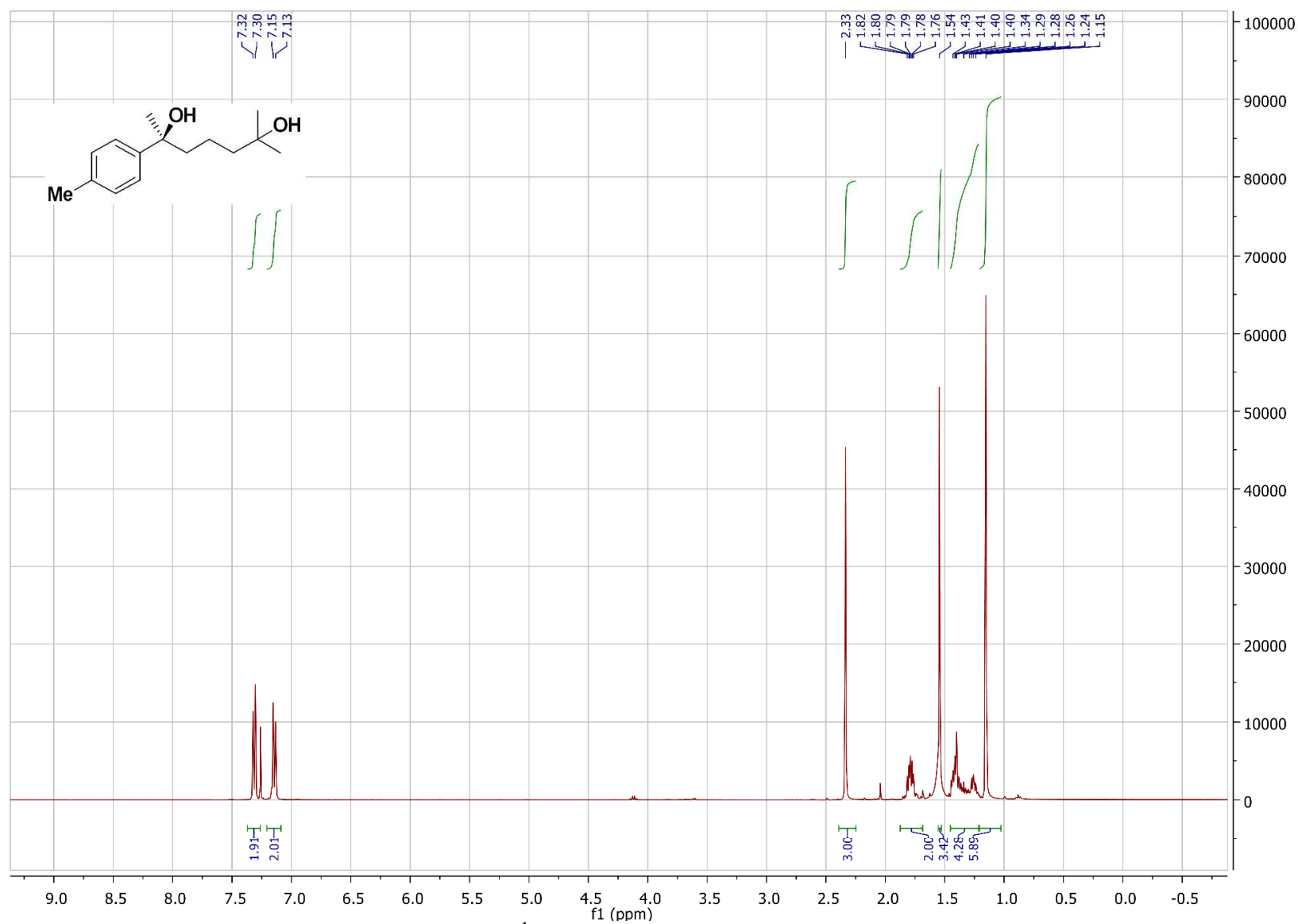

Figure S-55 ${ }^{1} \mathrm{H}-\mathrm{NMR}$ spectrum of compound $\mathbf{1 3}$.

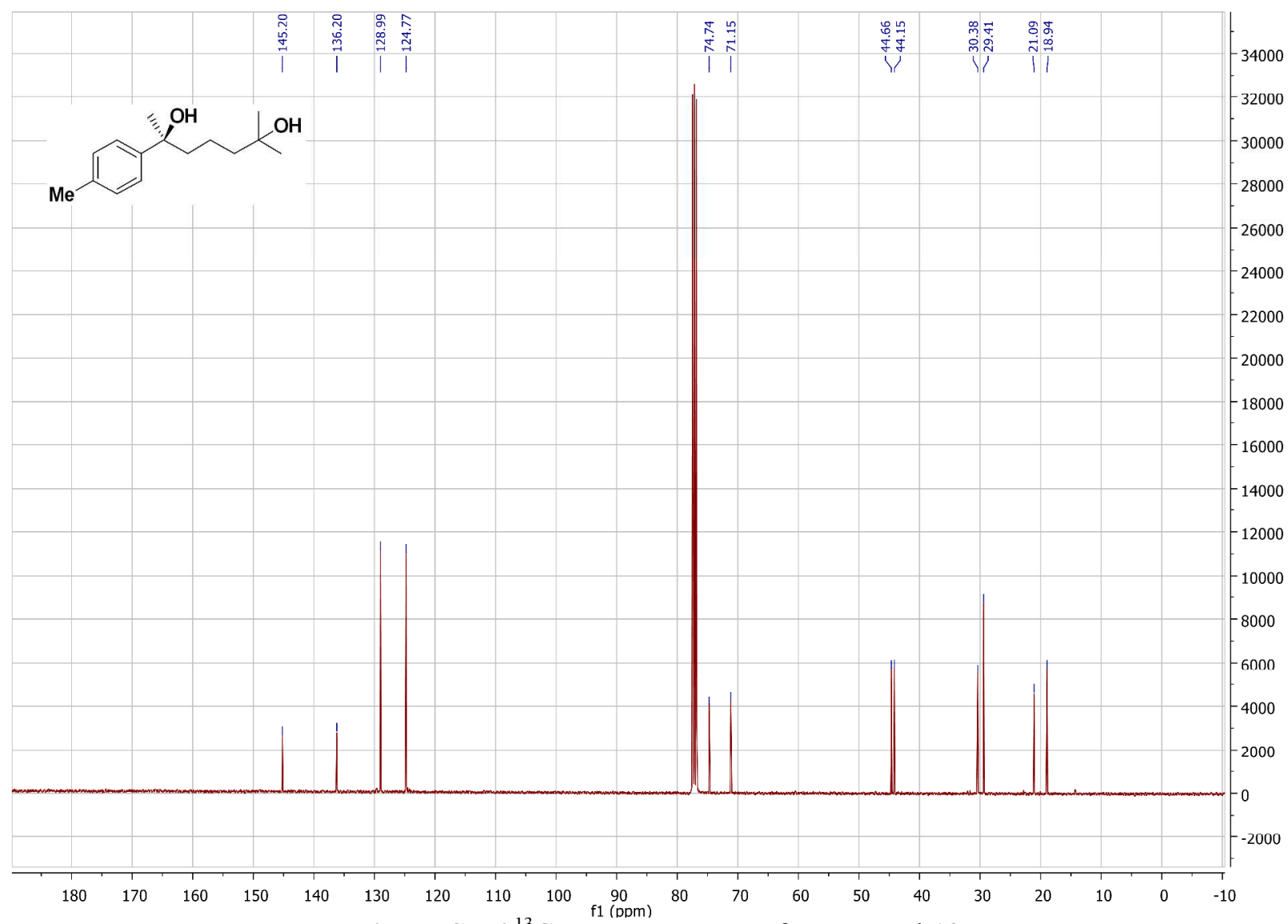

Figure S-56 ${ }^{13} \mathrm{C}-\mathrm{NMR}$ spectrum of compound $\mathbf{1 3}$. 


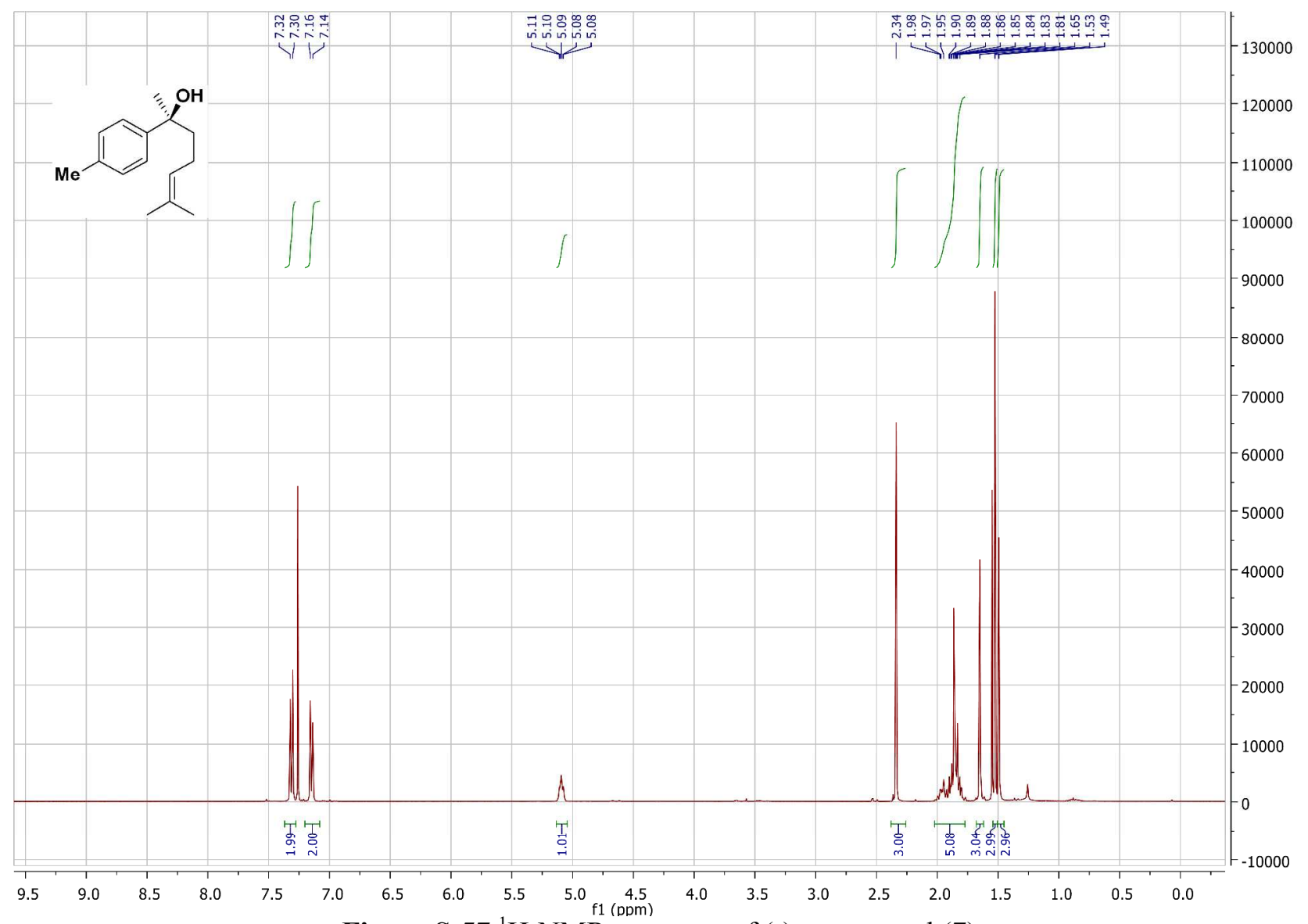

Figure S-57 ${ }^{1}$ H-NMR spectrum of (-)-gossonorol (7).

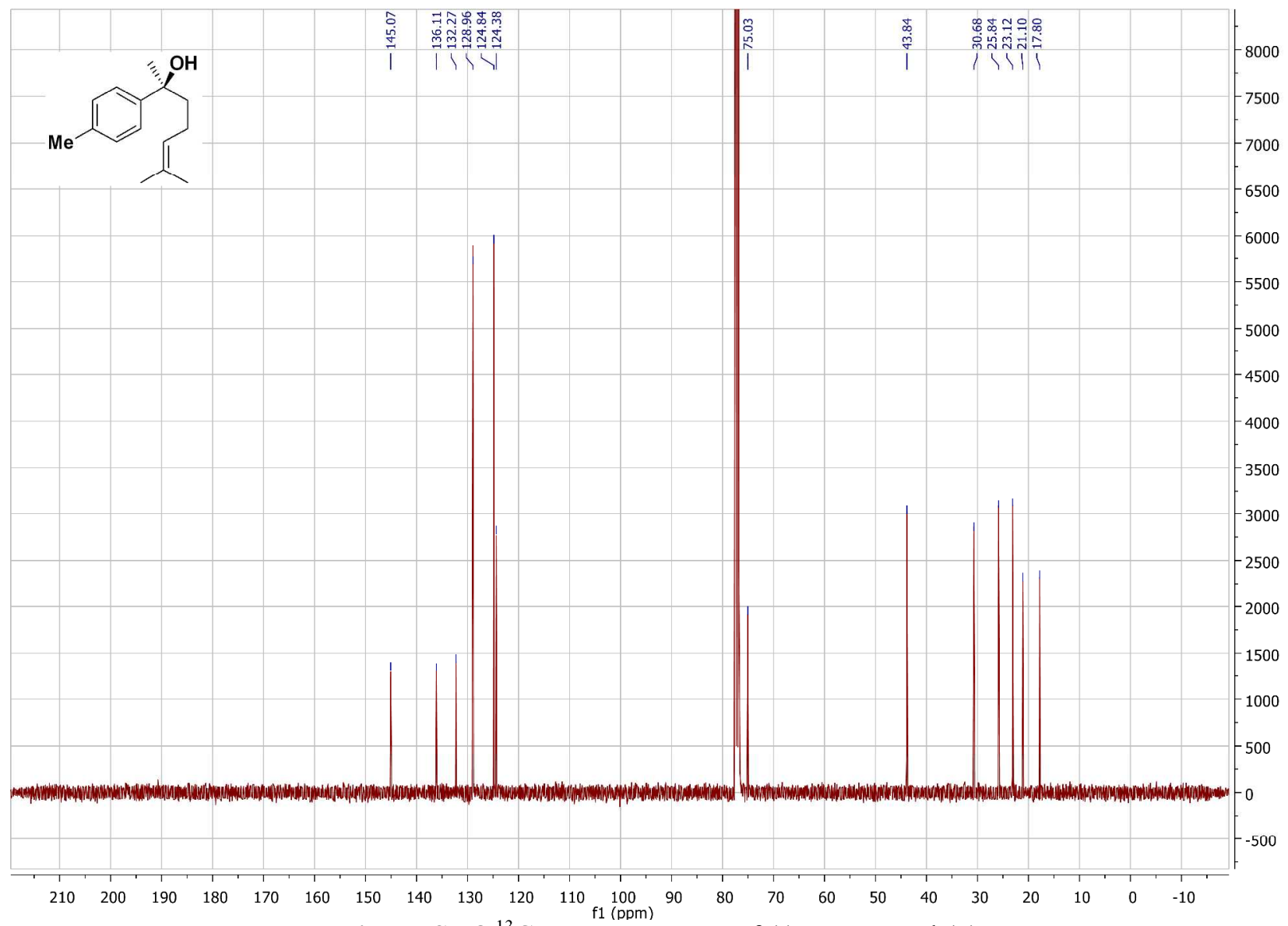

Figure S-58 ${ }^{13}$ C-NMR spectrum of (-)-gossonorol (7). 


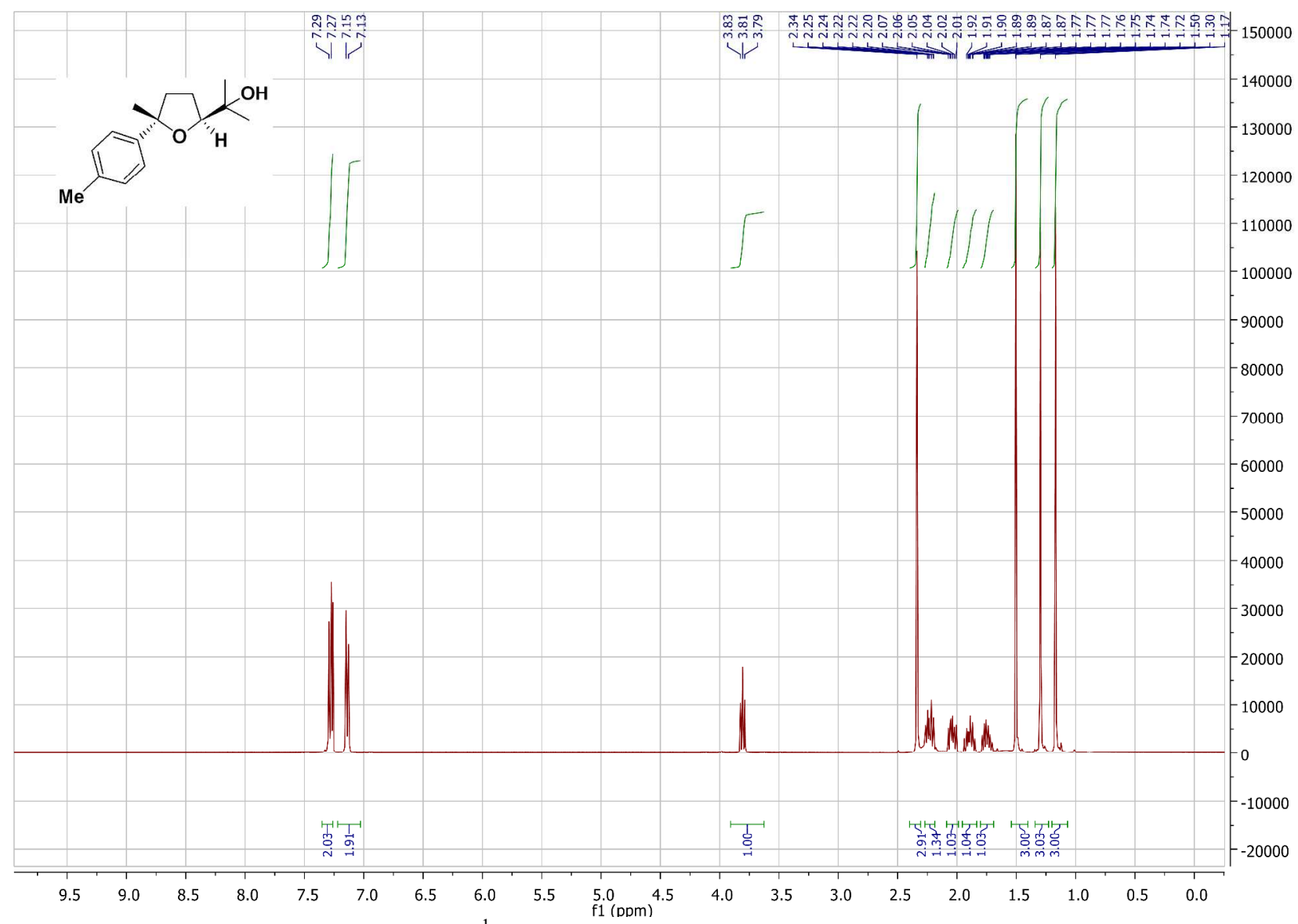

Figure S-59 ${ }^{1}$ H-NMR spectrum of (-)-boivinianin B (9).

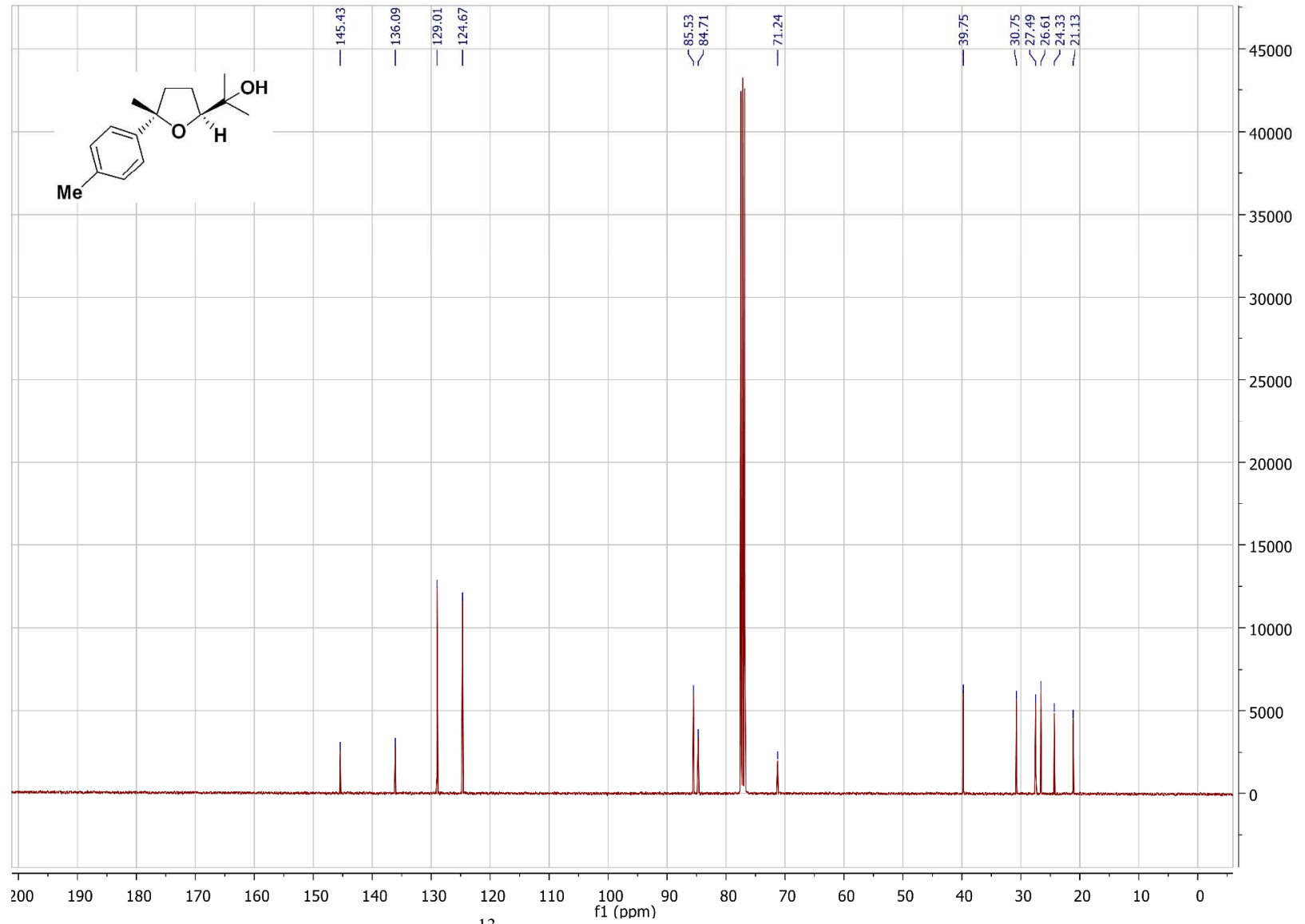

Figure S-60 ${ }^{13} \mathrm{C}-\mathrm{NMR}$ spectrum of (-)-boivinianin B (9). 
(mdd) It

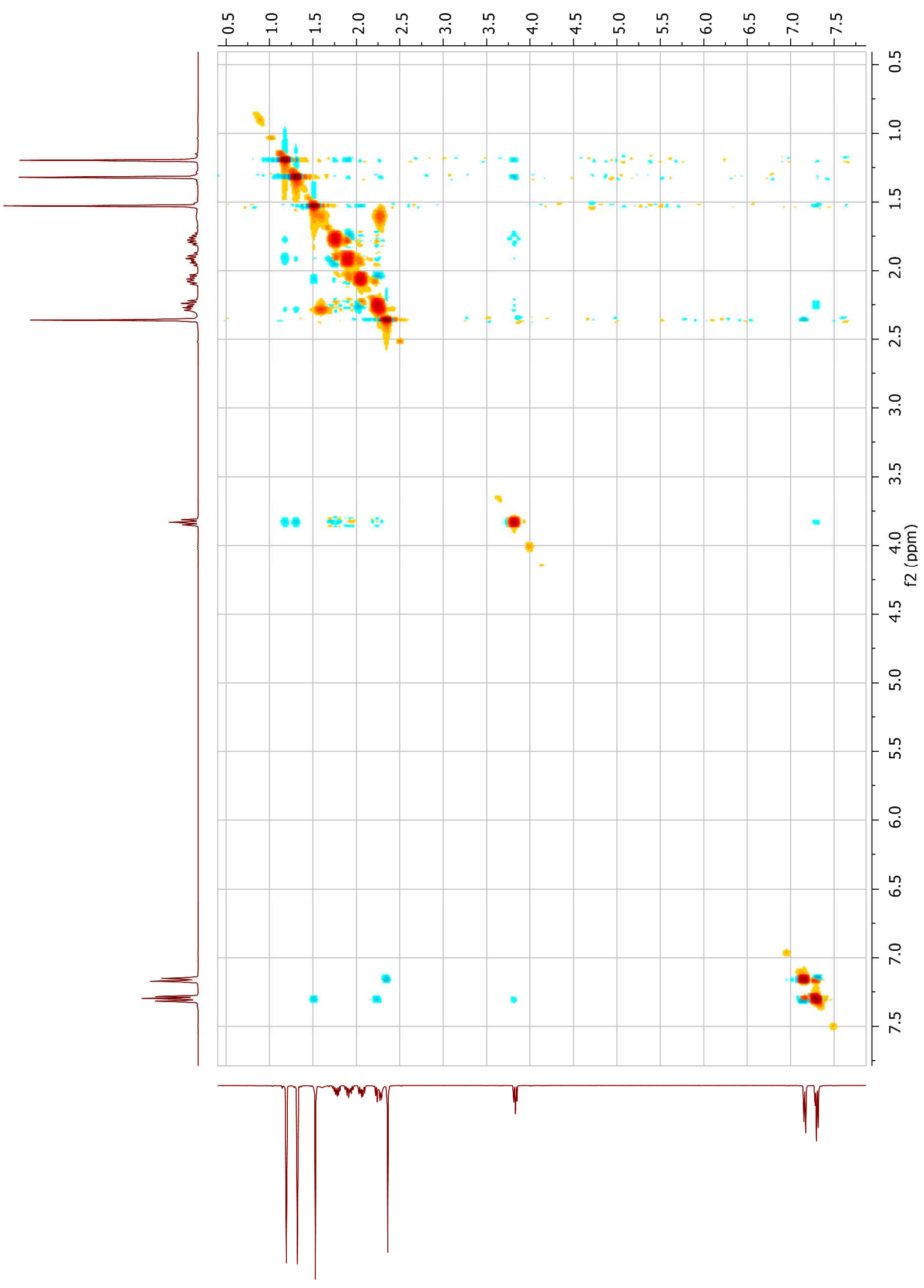

Figure S-61 NOESY spectrum of (-)-boivinianin B (9). 


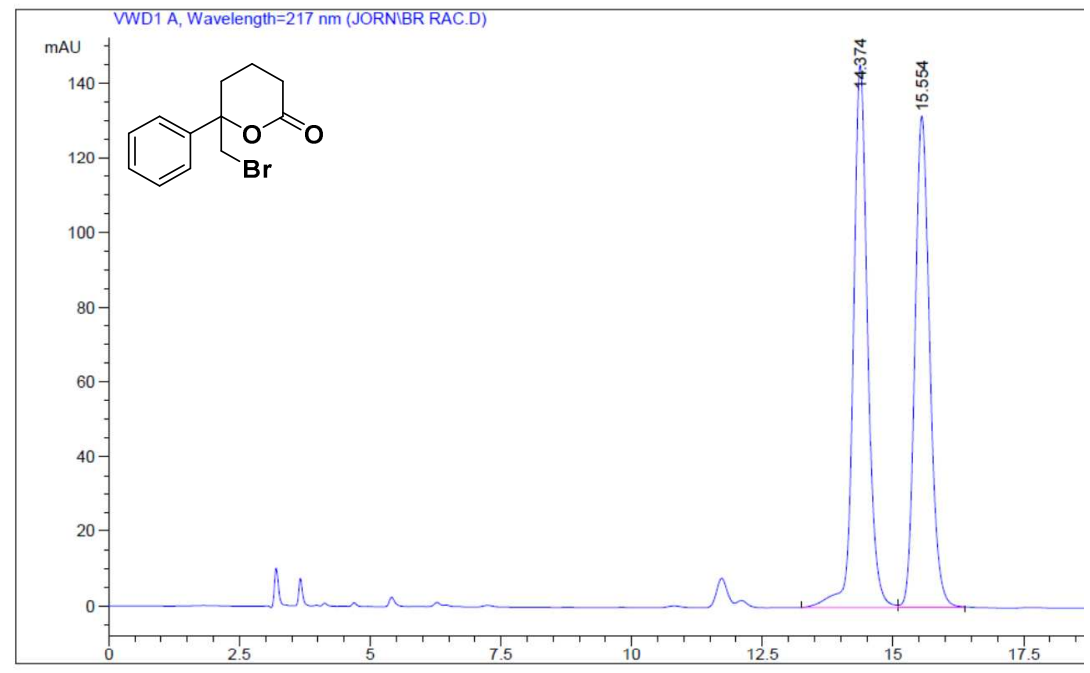

Area Percent Report

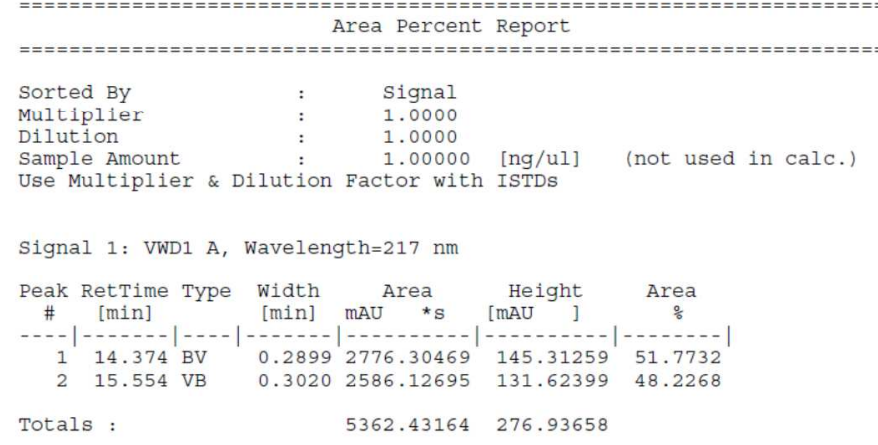

Figure S-62 HPLC chromatogram of racemic compound 6a.

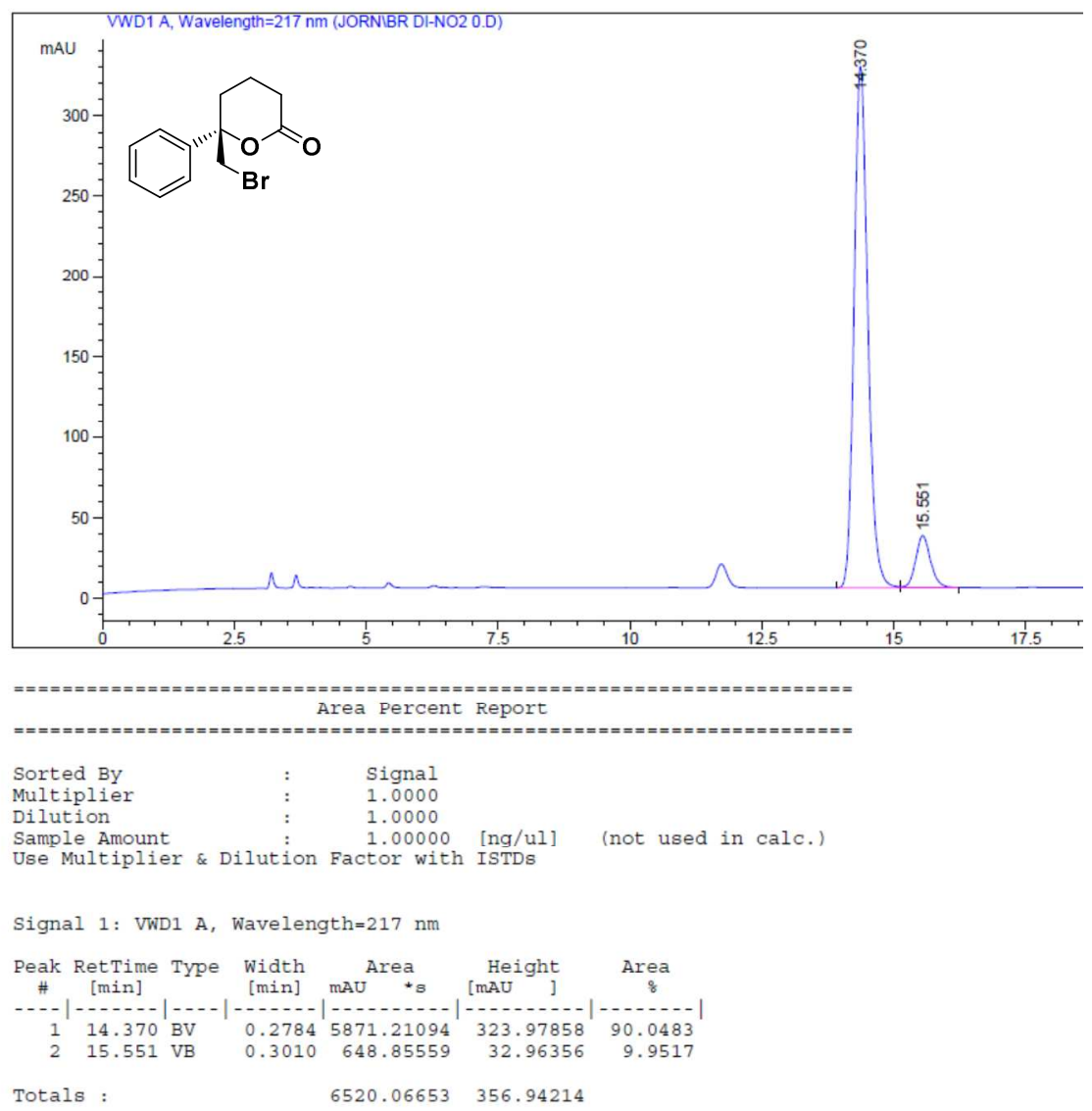

Figure S-63 HPLC chromatogram of compound 6a. 


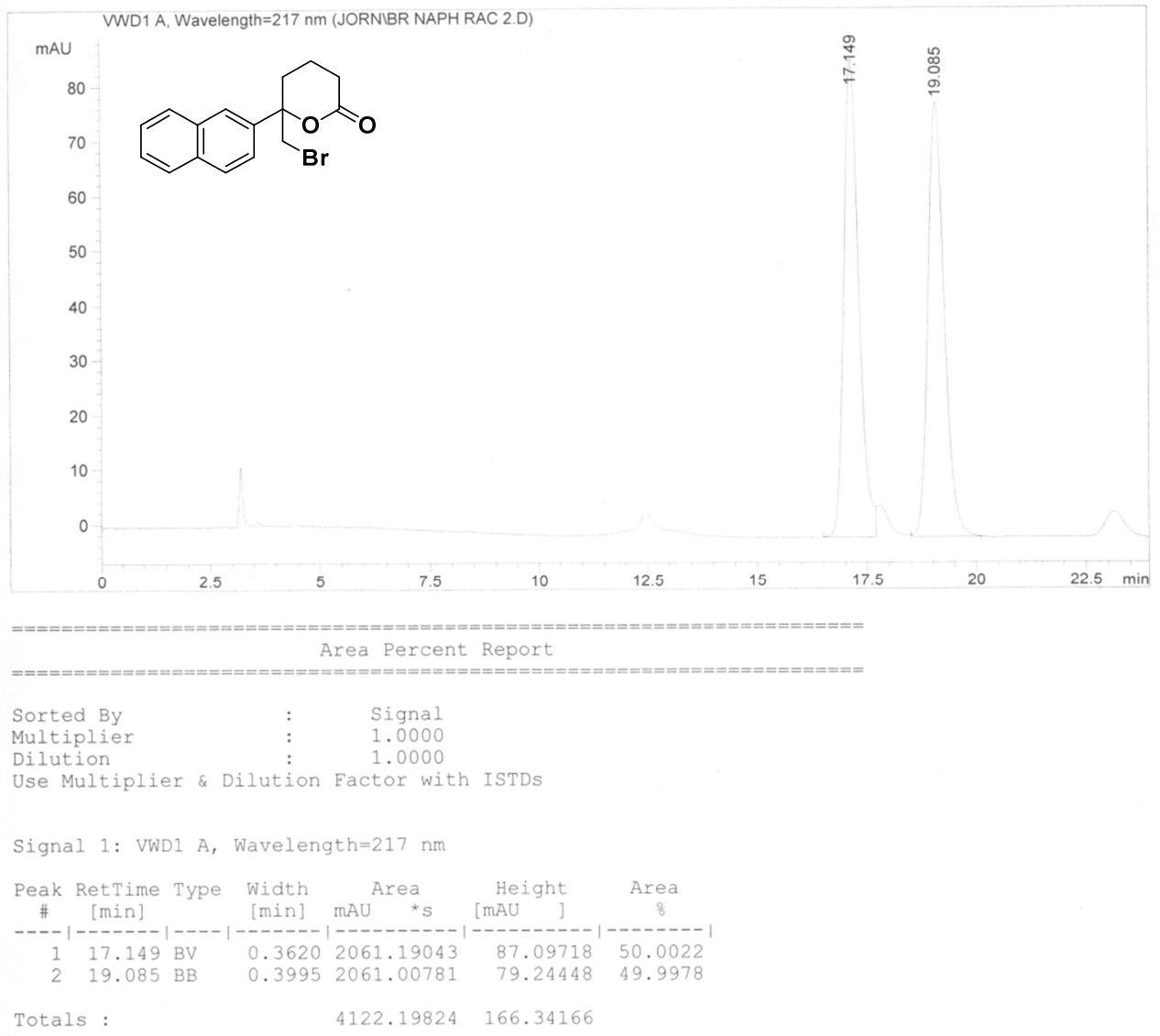

Figure S-64 HPLC chromatogram of racemic compound $\mathbf{6 b}$.

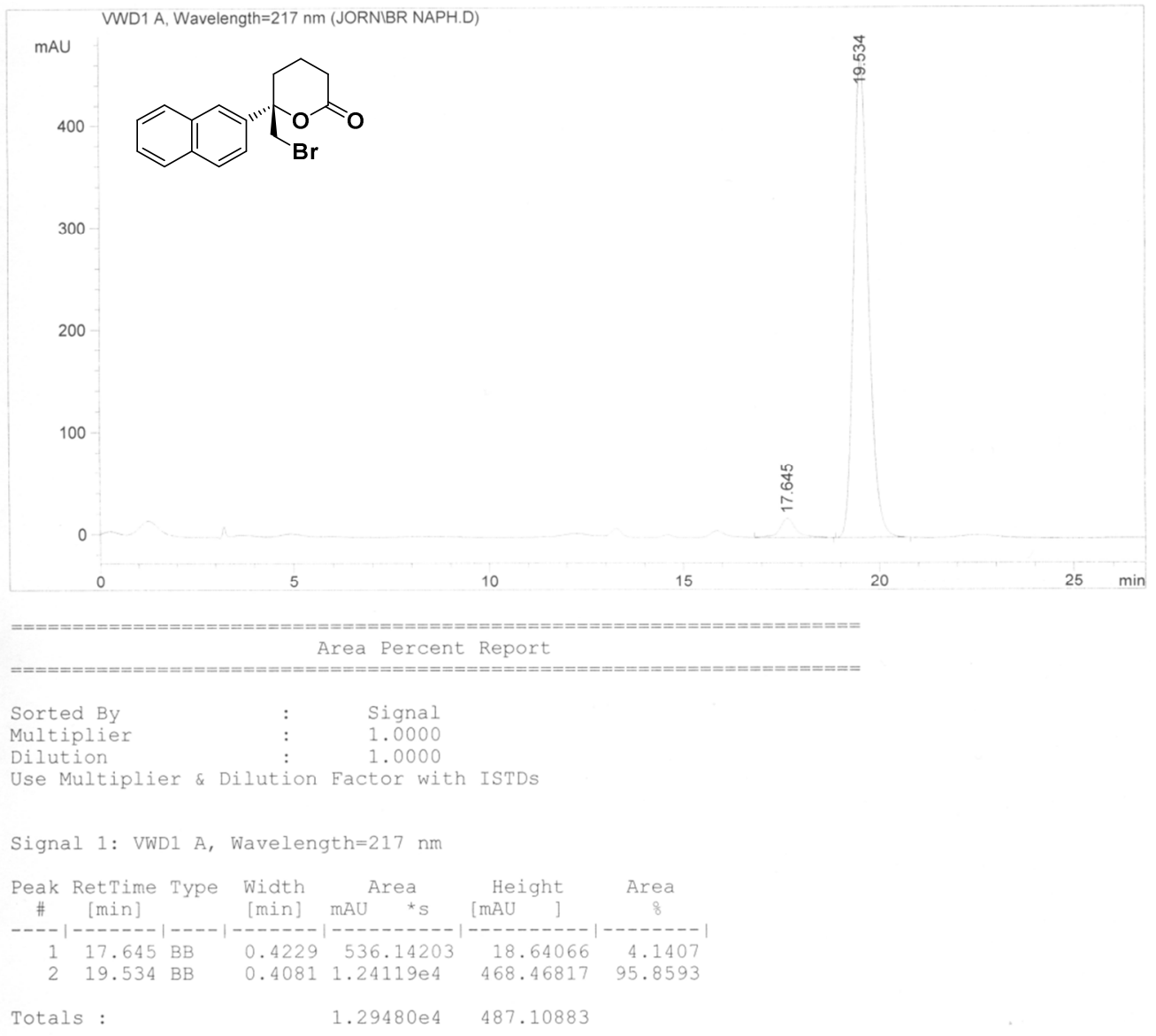

Figure S-65 HPLC chromatogram of compound $\mathbf{6 b}$. 


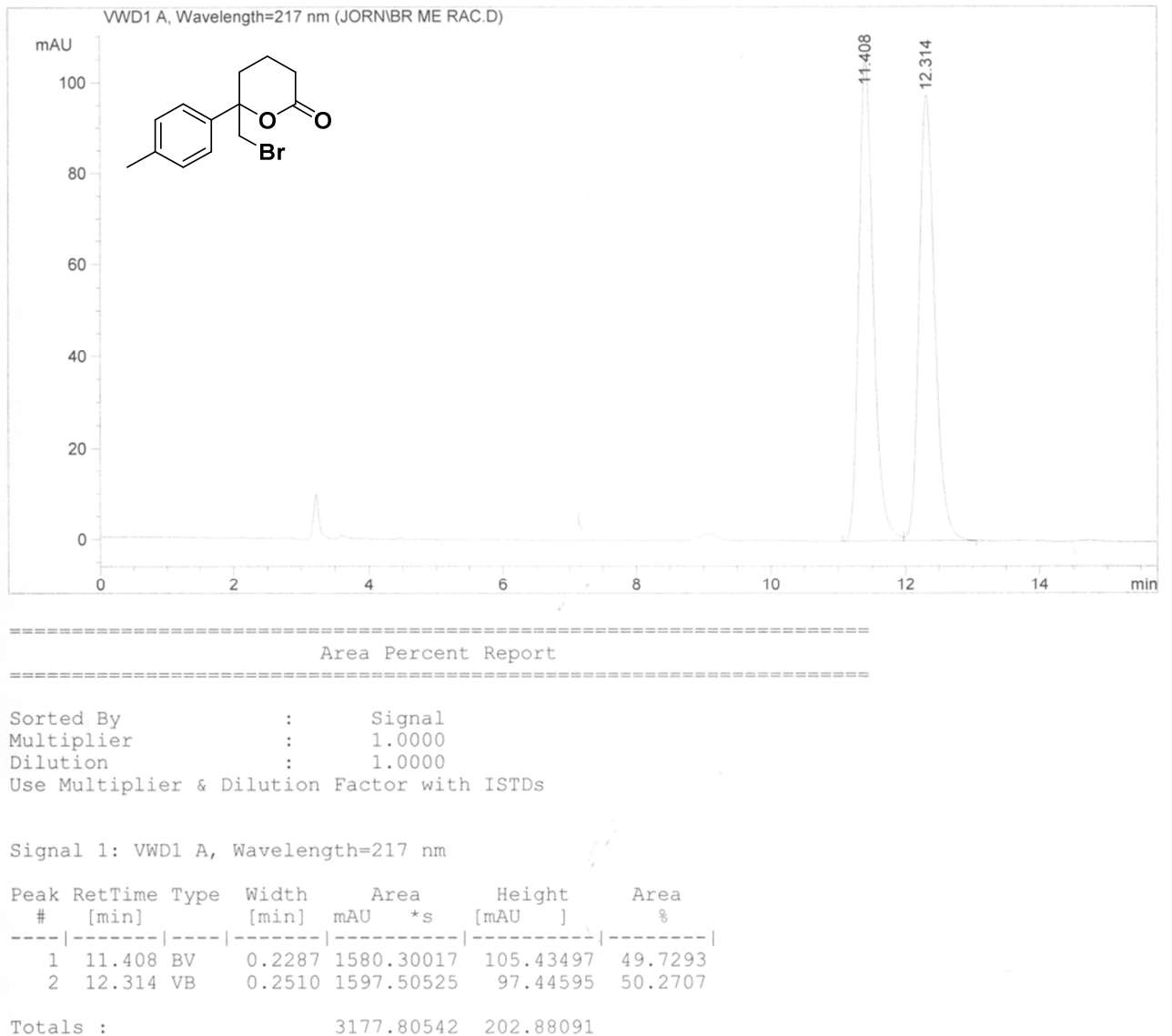

Figure S-66 HPLC chromatogram of racemic compound 6c.

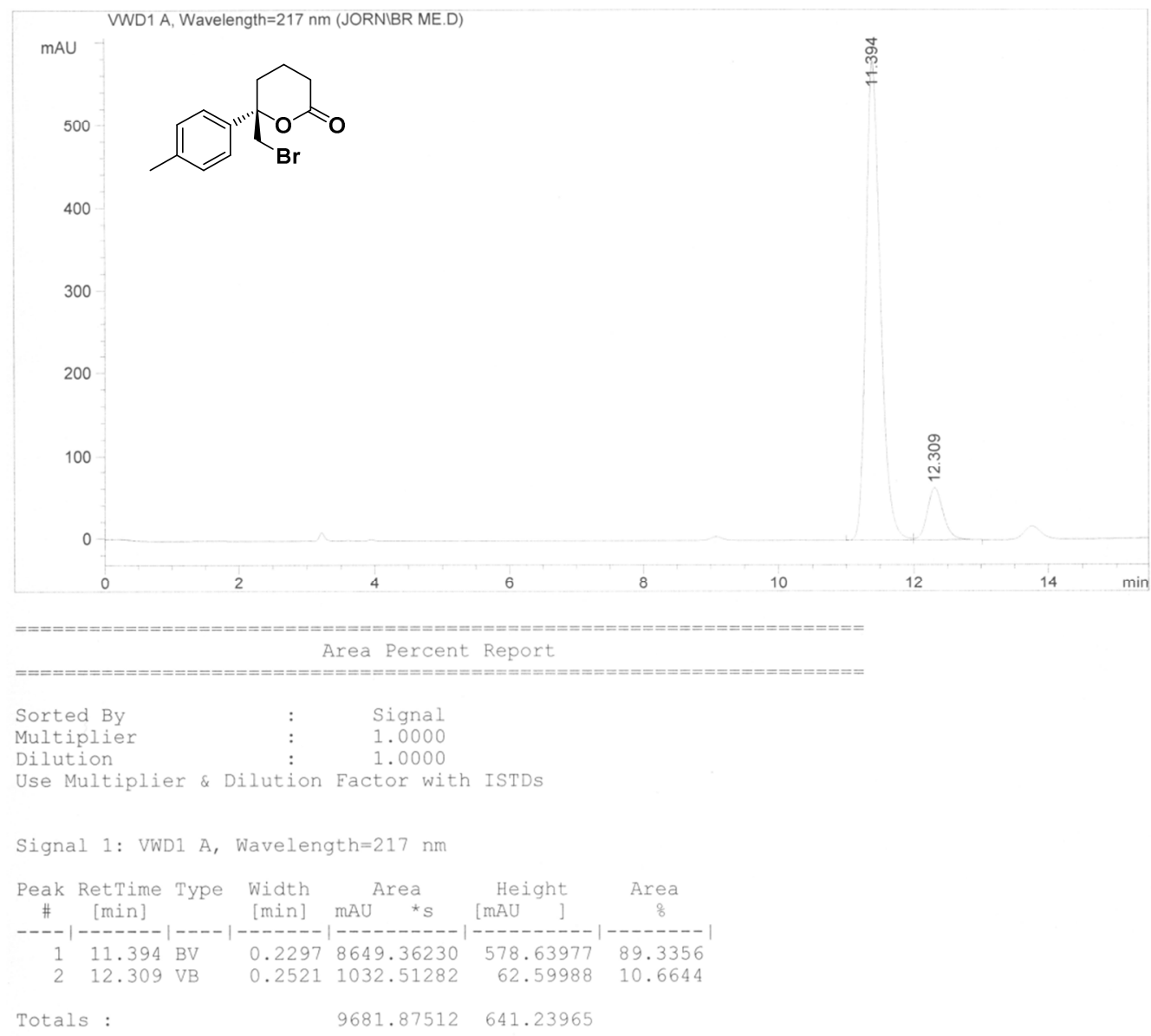

Figure S-67 HPLC chromatogram of compound 6c. 


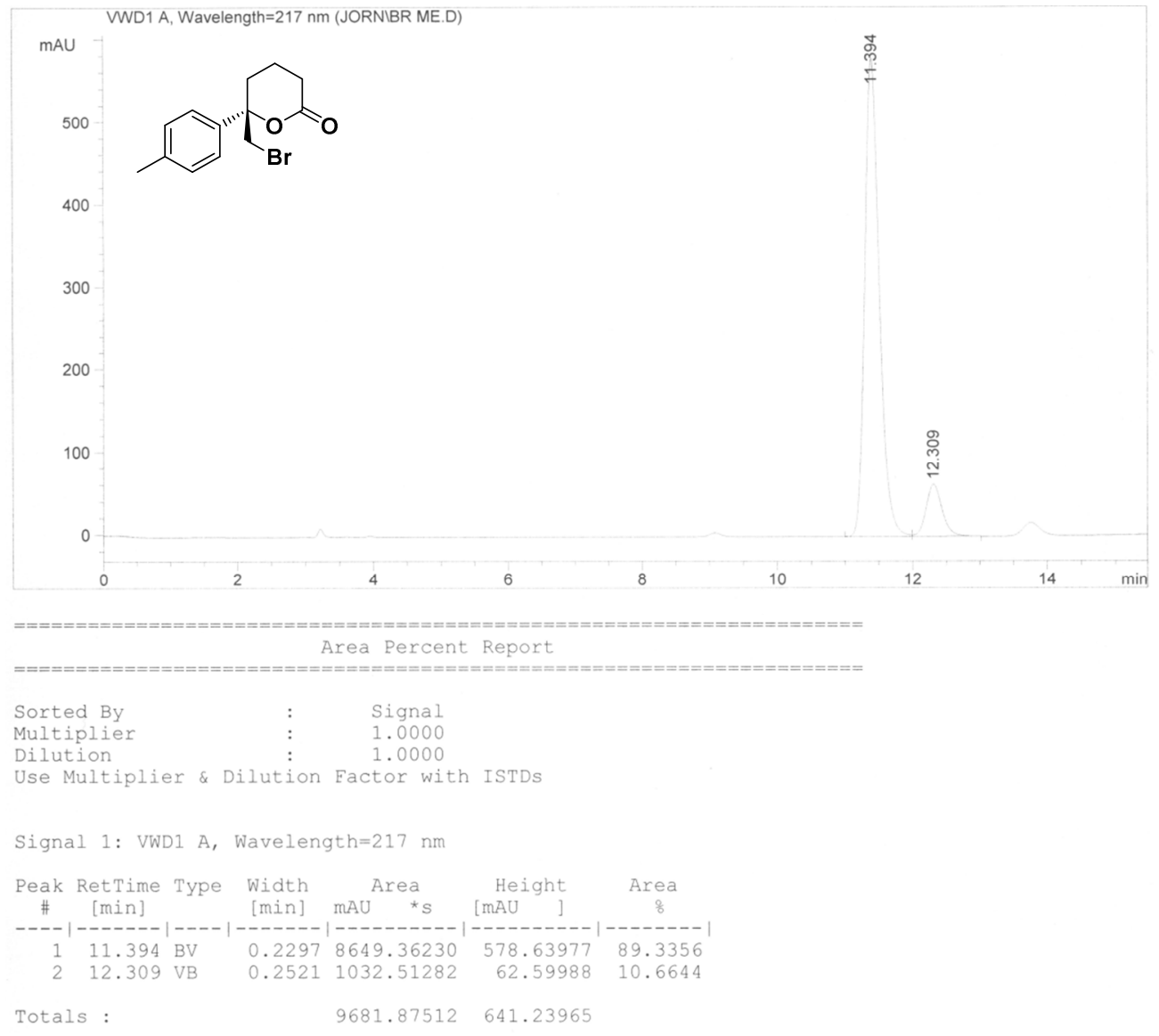

Figure S-68 HPLC chromatogram of big scale preparation of compound $\mathbf{6 c}$.

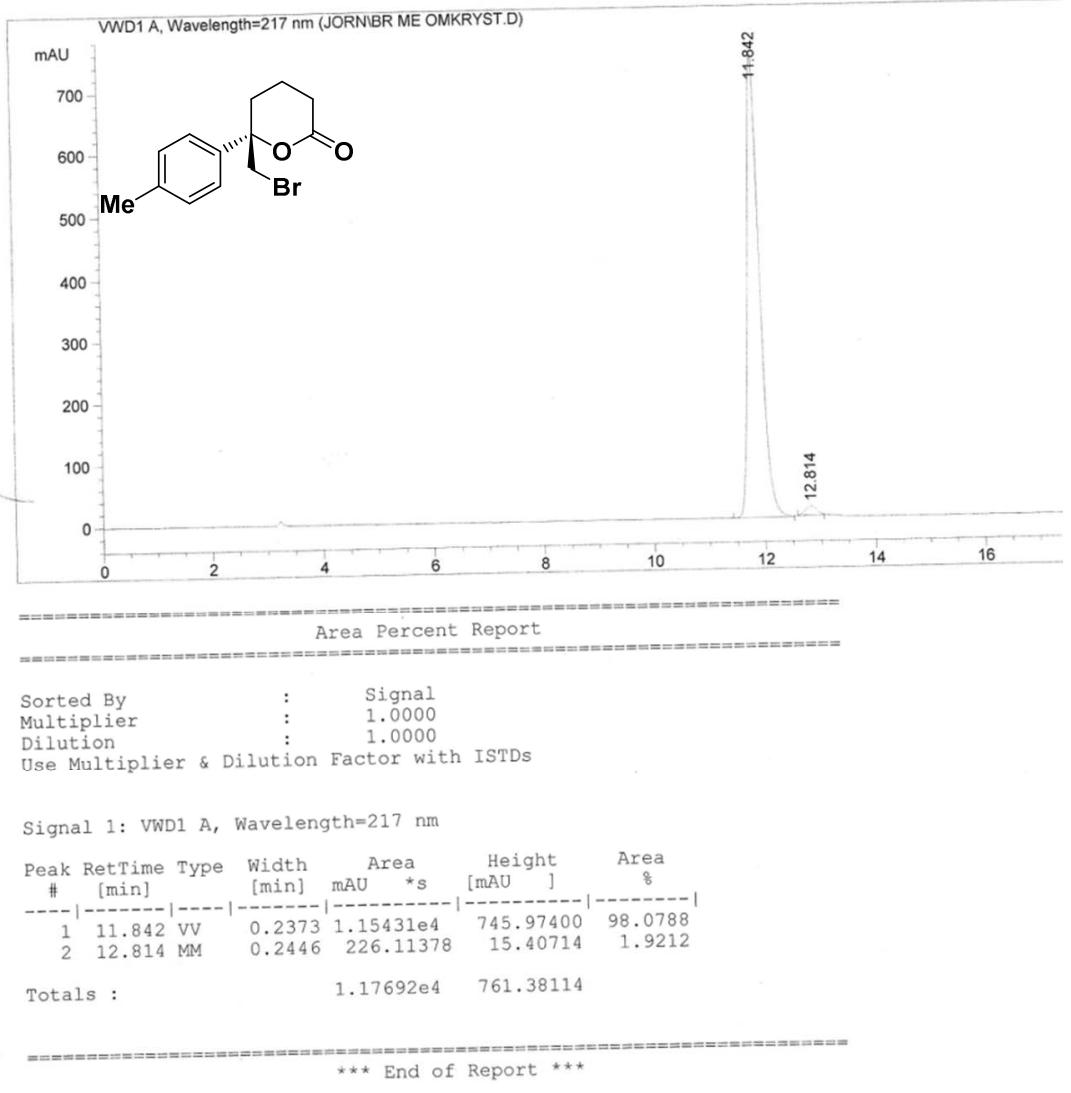

Figure S-69 HPLC chromatogram of recrystallized compound $\mathbf{6 c}$. 


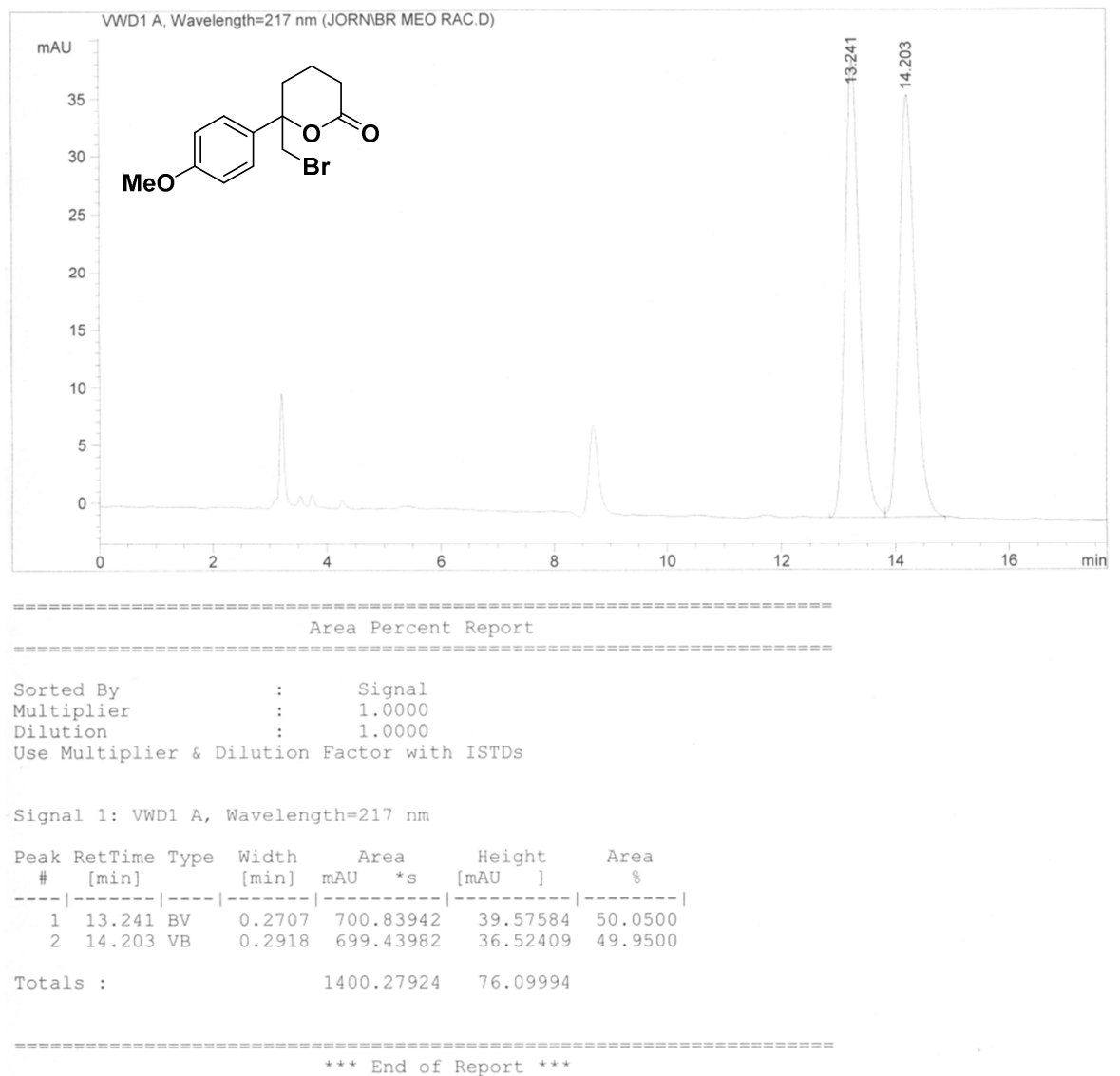

Figure S-70 HPLC chromatogram of racemic compound $6 \mathbf{d}$.

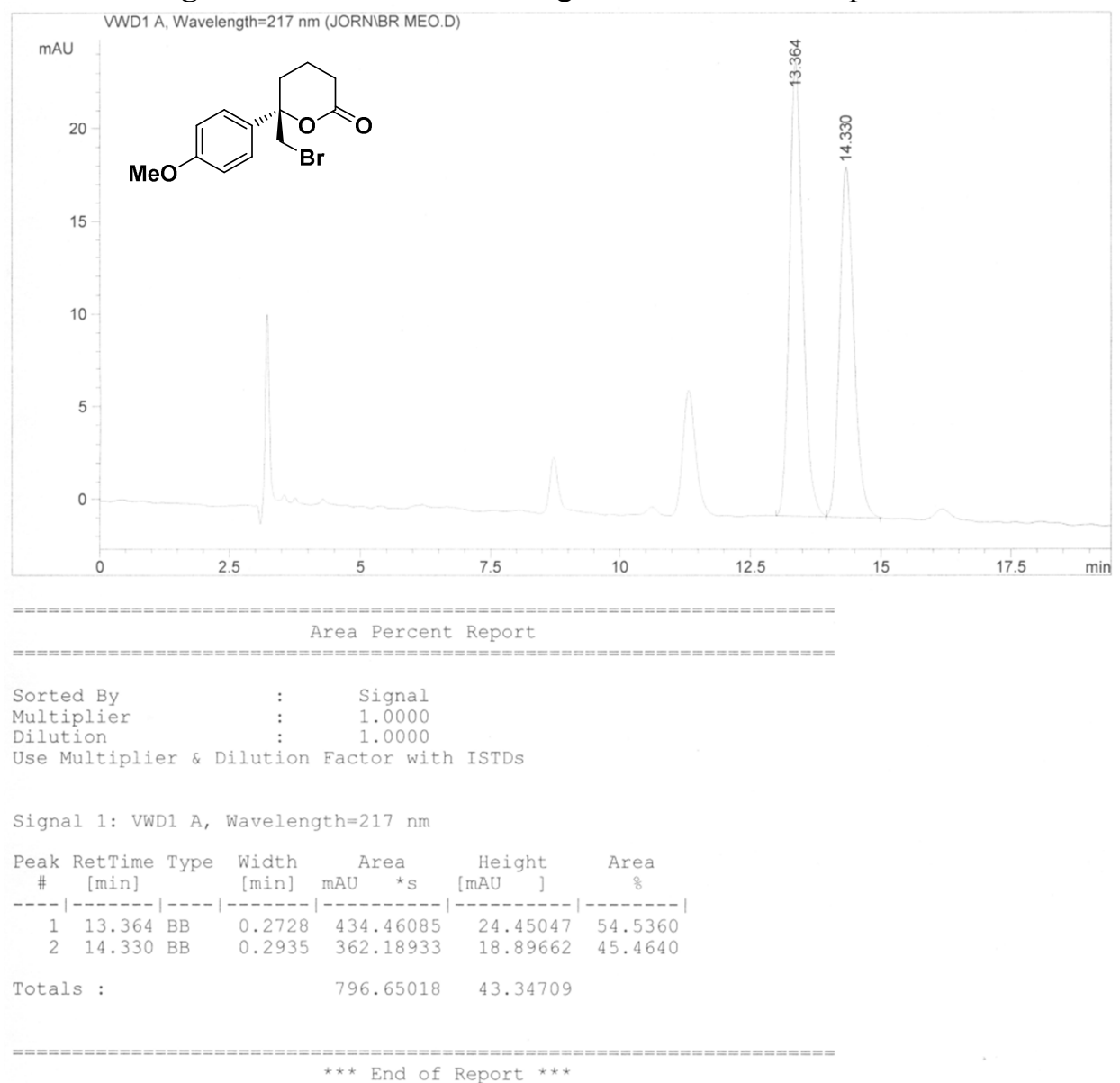

Figure S-71 HPLC chromatogram of compound $\mathbf{6 d}$. 


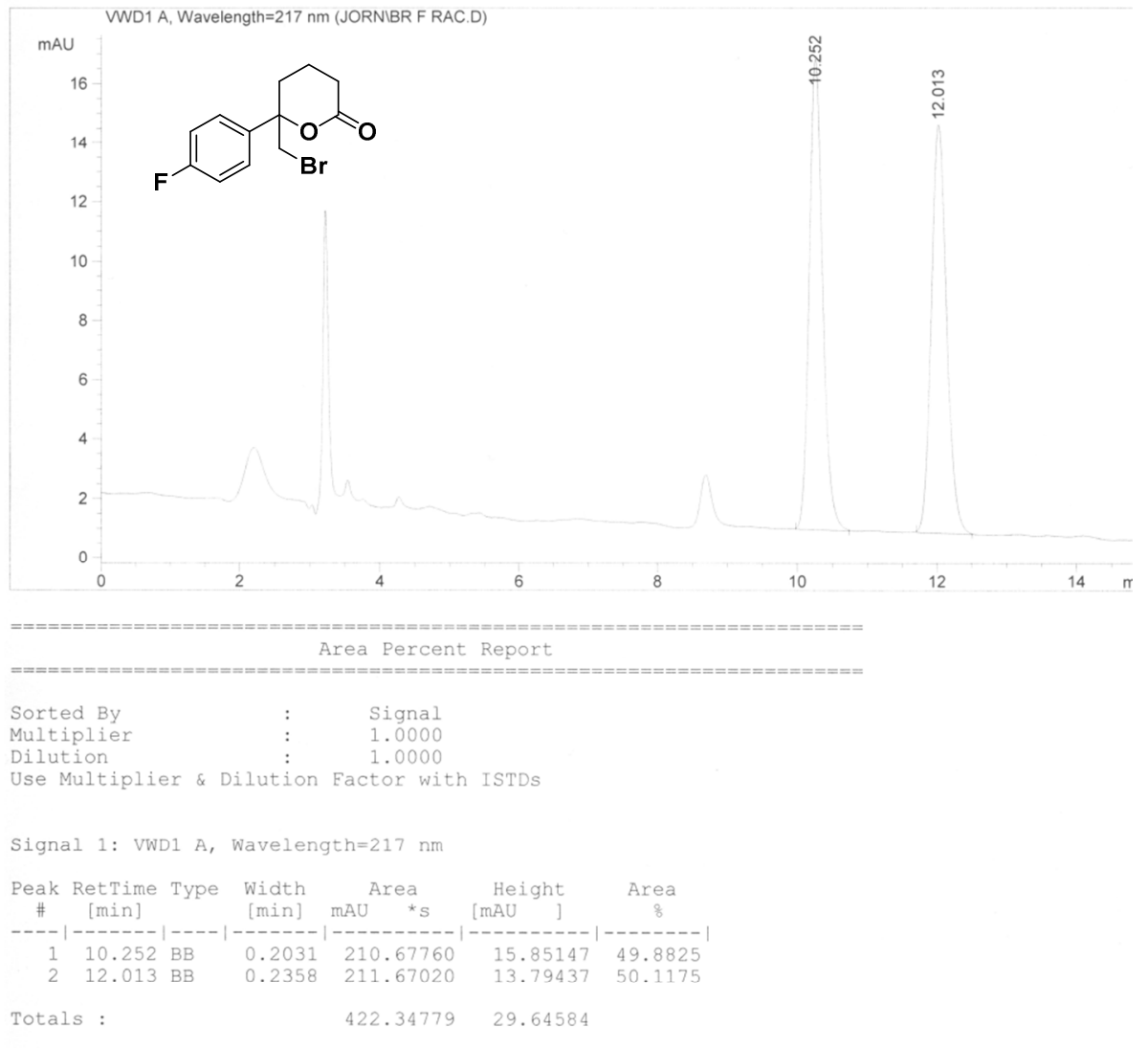

Figure S-72 HPLC chromatogram of racemic compound 6 e.

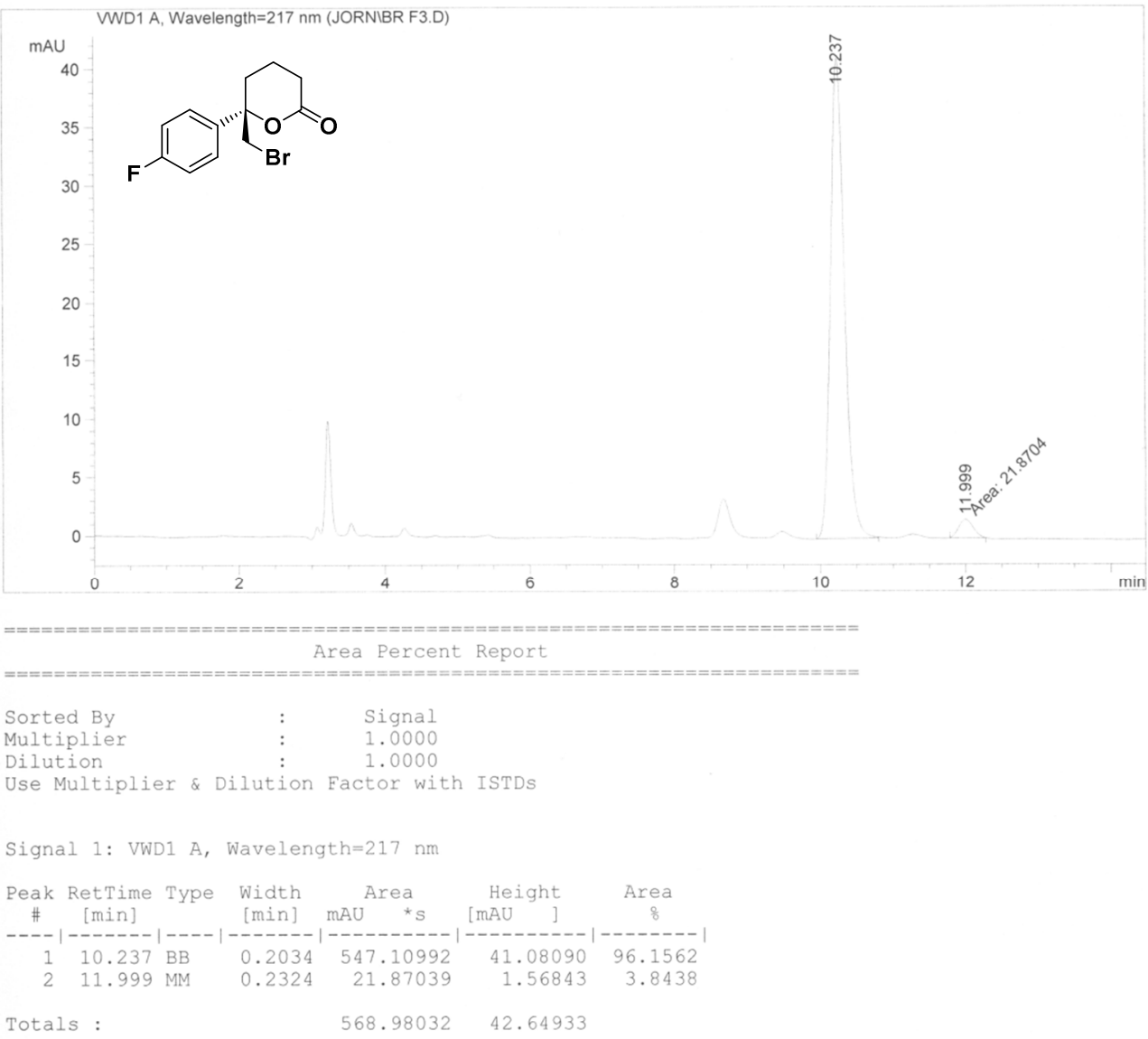

Figure S-73 HPLC chromatogram of compound $\mathbf{6 e}$. 


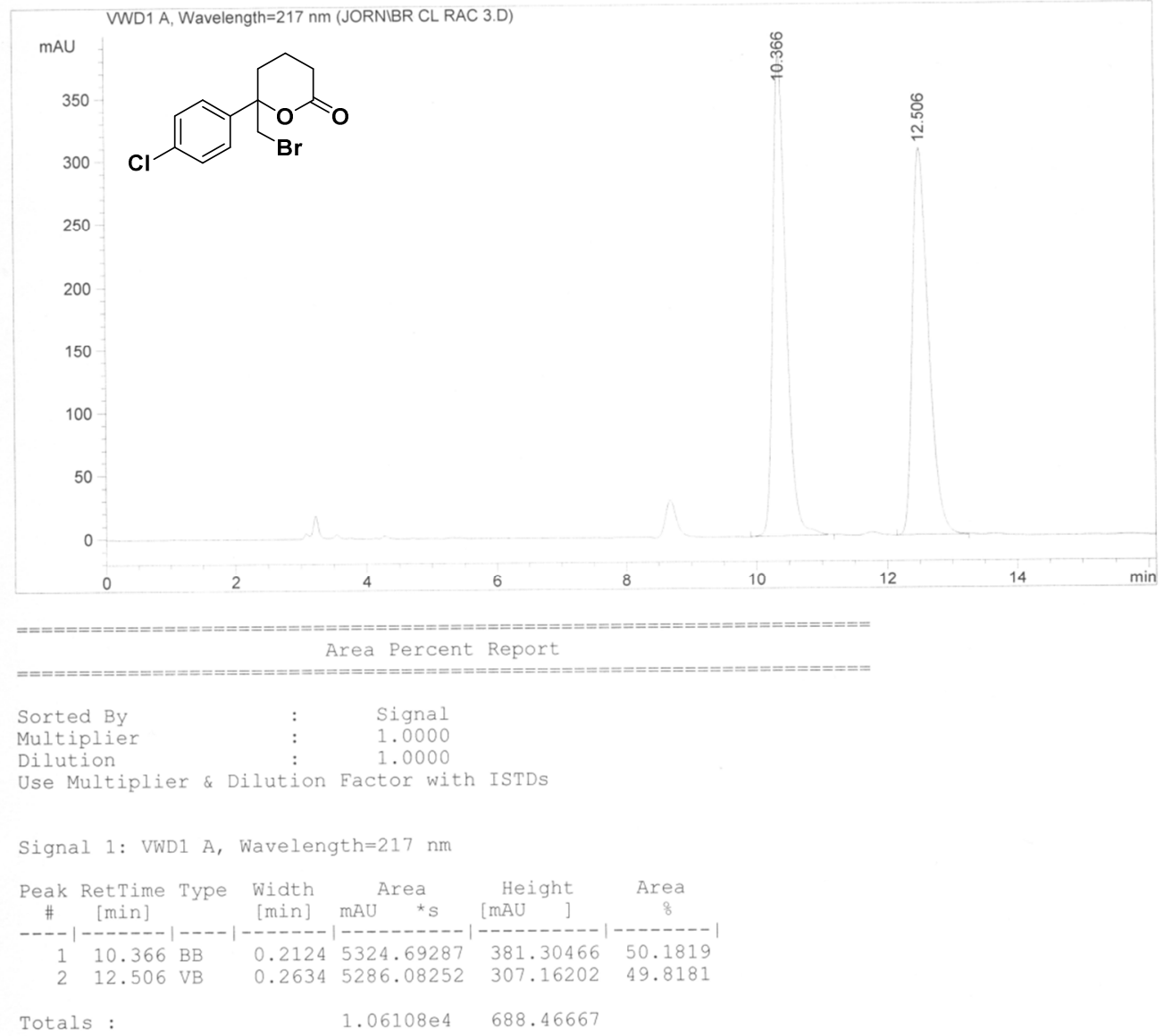

Figure S-74 HPLC chromatogram of racemic compound $\mathbf{6 f}$.

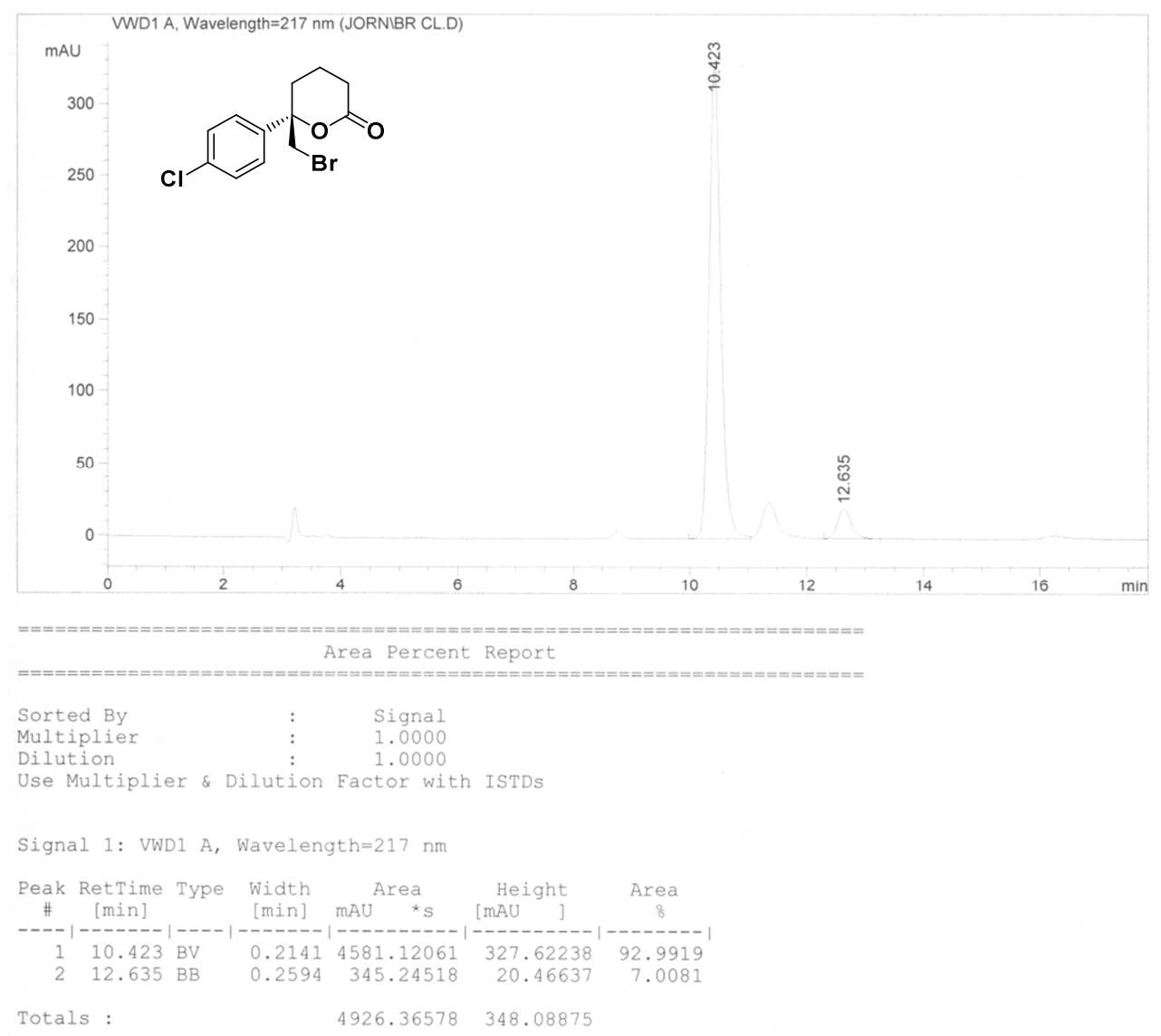

Figure S-75 HPLC chromatogram of compound $\mathbf{6 f}$. 


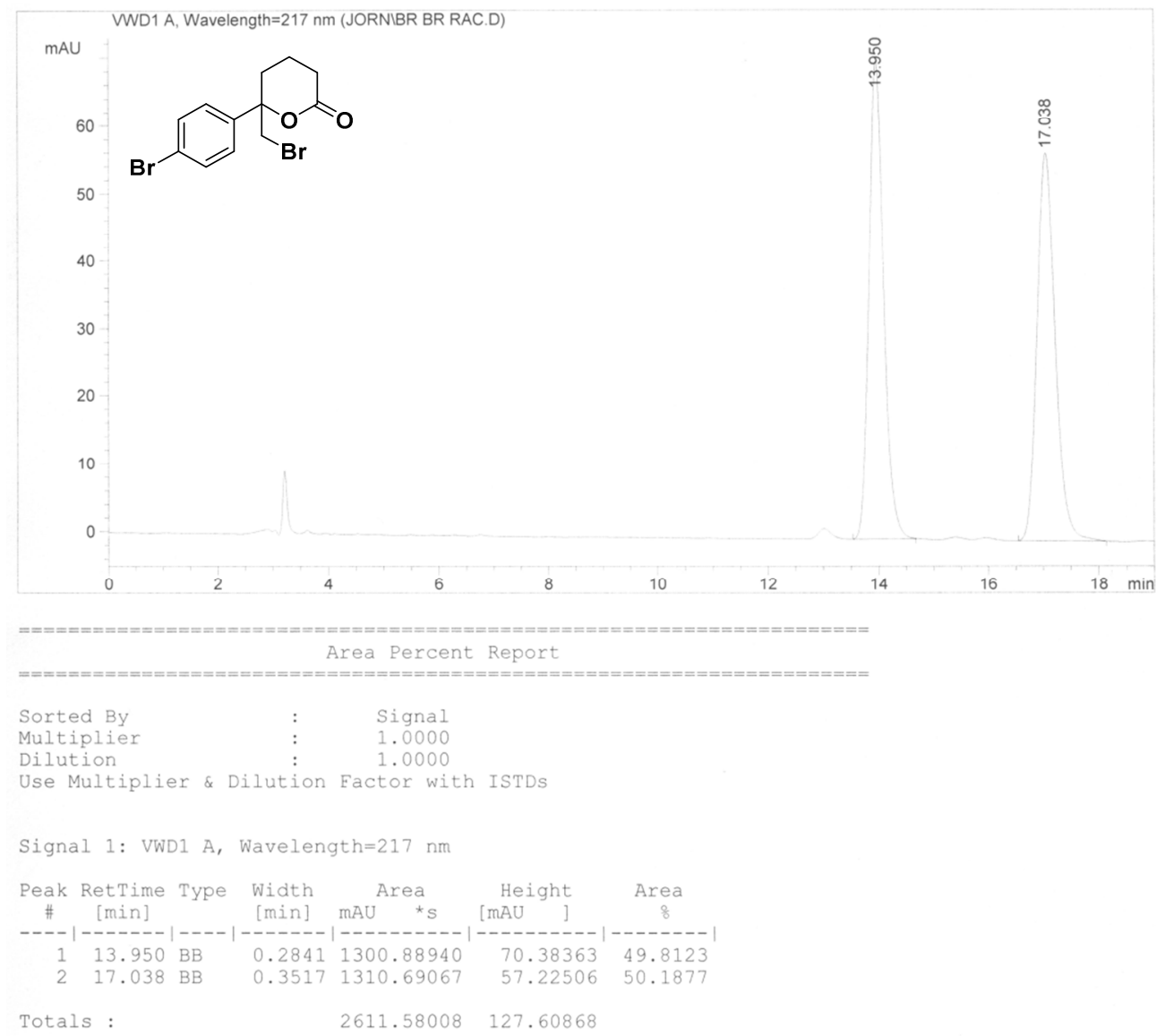

Figure S-76 HPLC chromatogram of racemic compound $\mathbf{6 g}$.

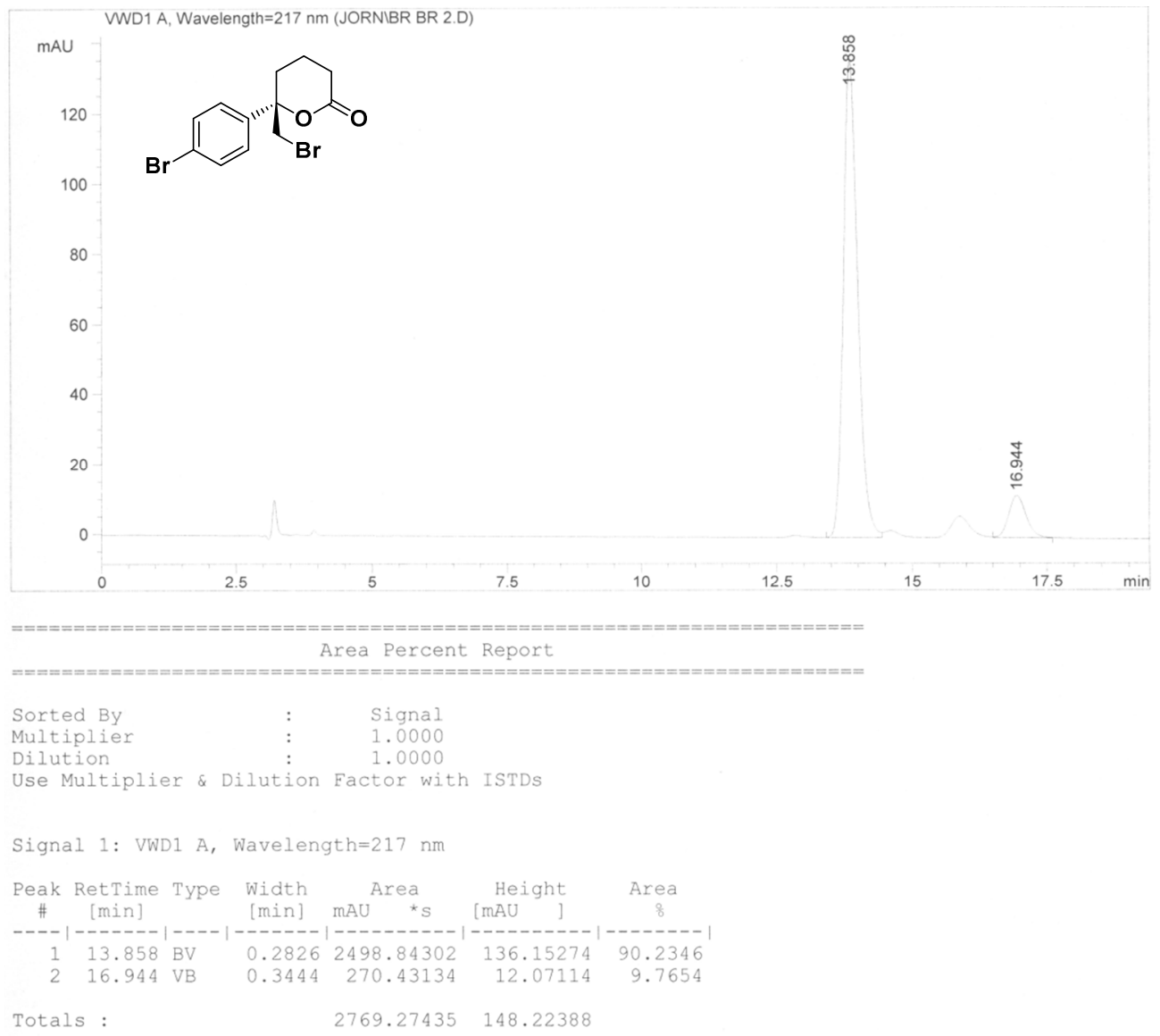

Figure S-77 HPLC chromatogram of compound $\mathbf{6 g}$. 


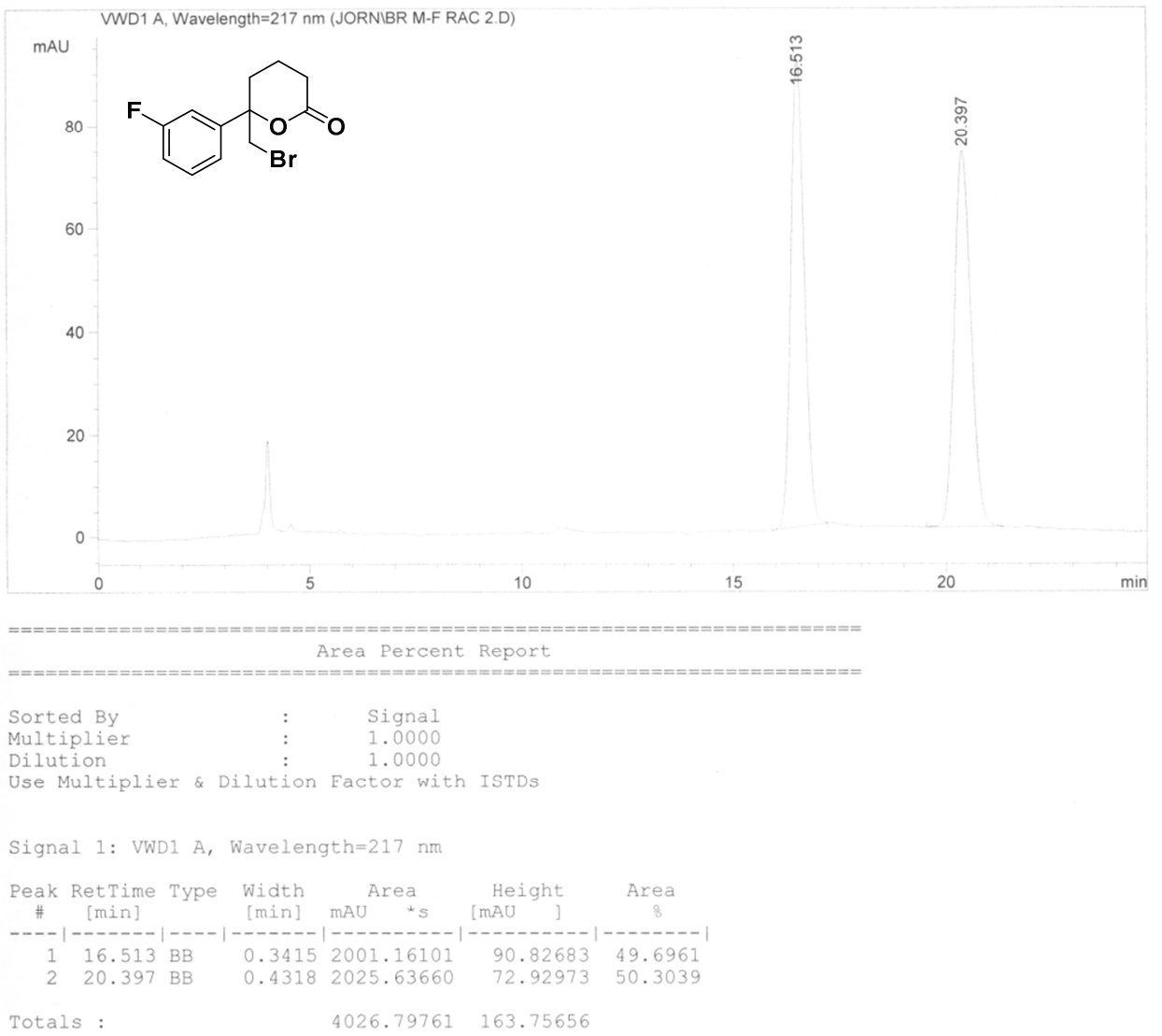

Figure S-78 HPLC chromatogram of racemic compound $6 \mathbf{h}$.

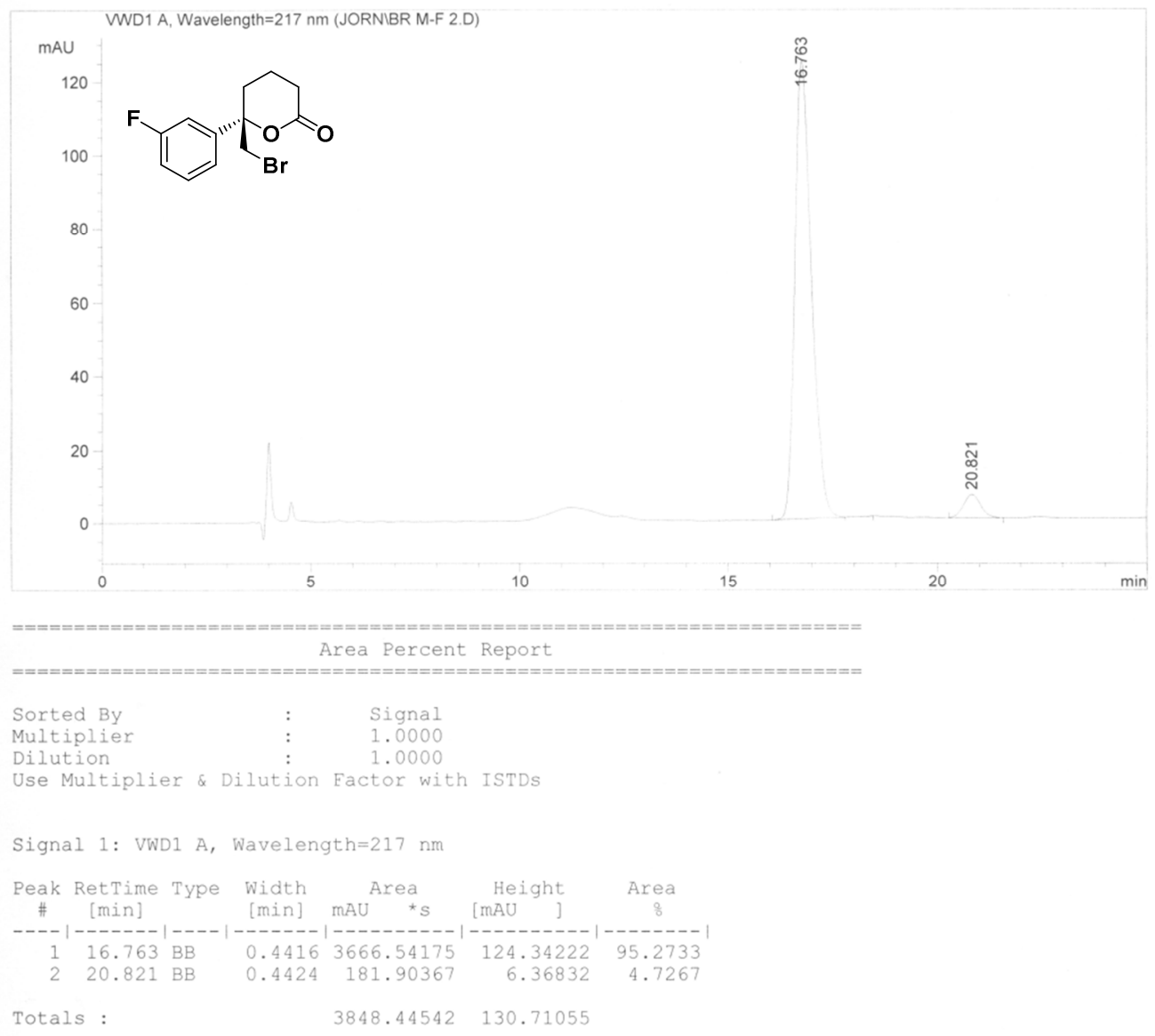

Figure S-79 HPLC chromatogram of compound $6 \mathbf{h}$. 


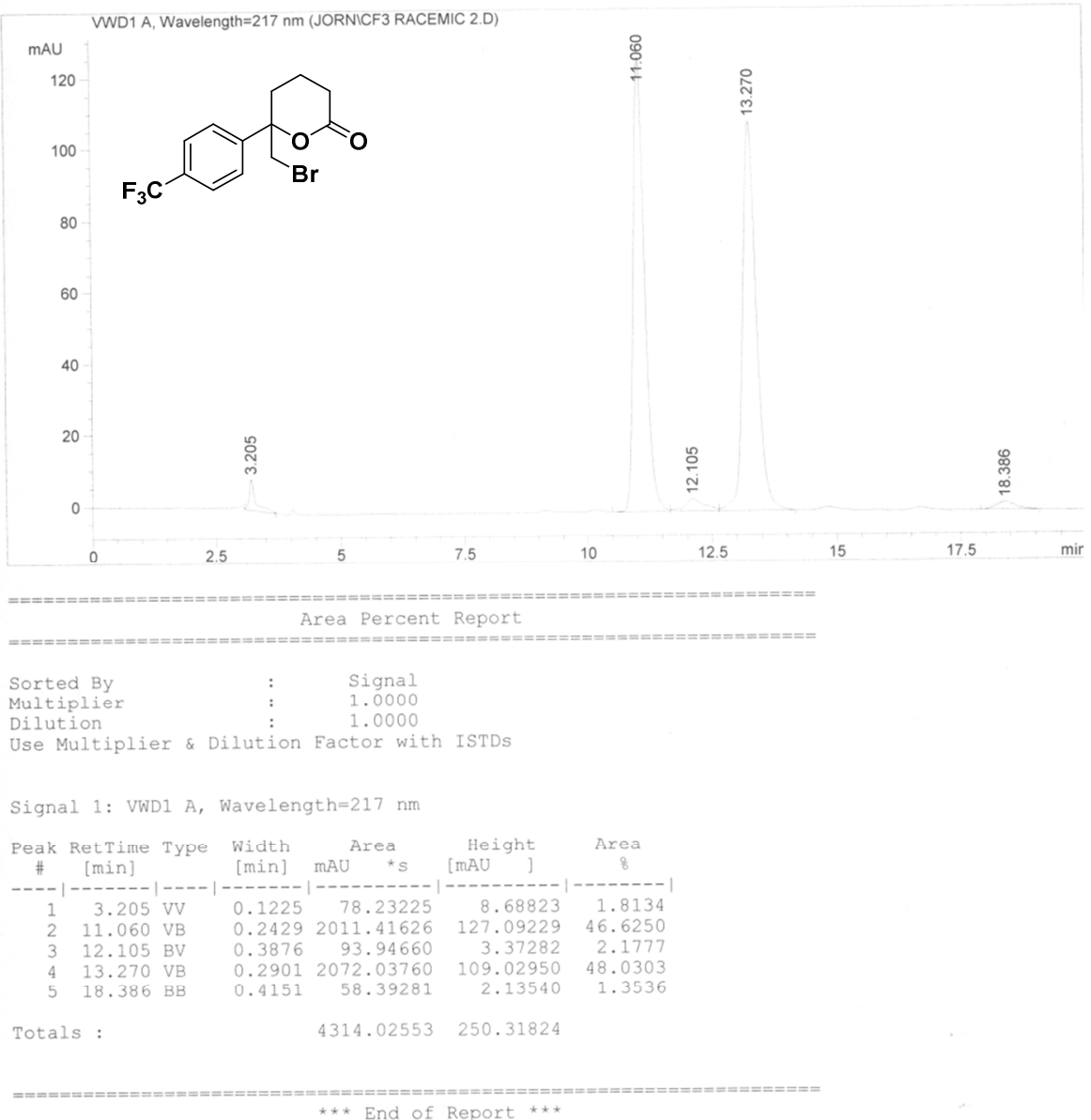

Figure S-80 HPLC chromatogram of racemic compound $\mathbf{6 i}$.

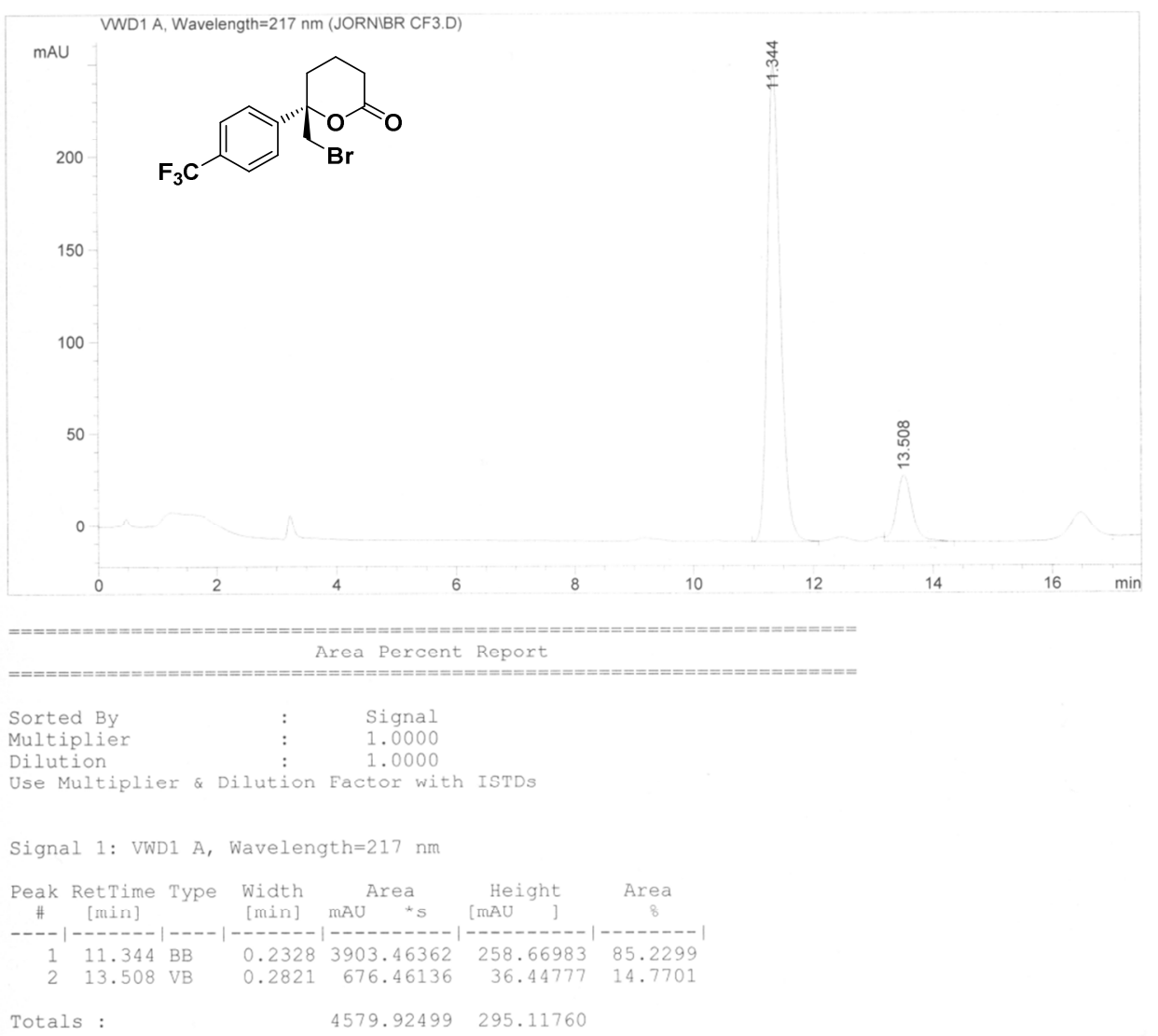

Figure S-81 HPLC chromatogram of compound $6 \mathbf{i}$. 


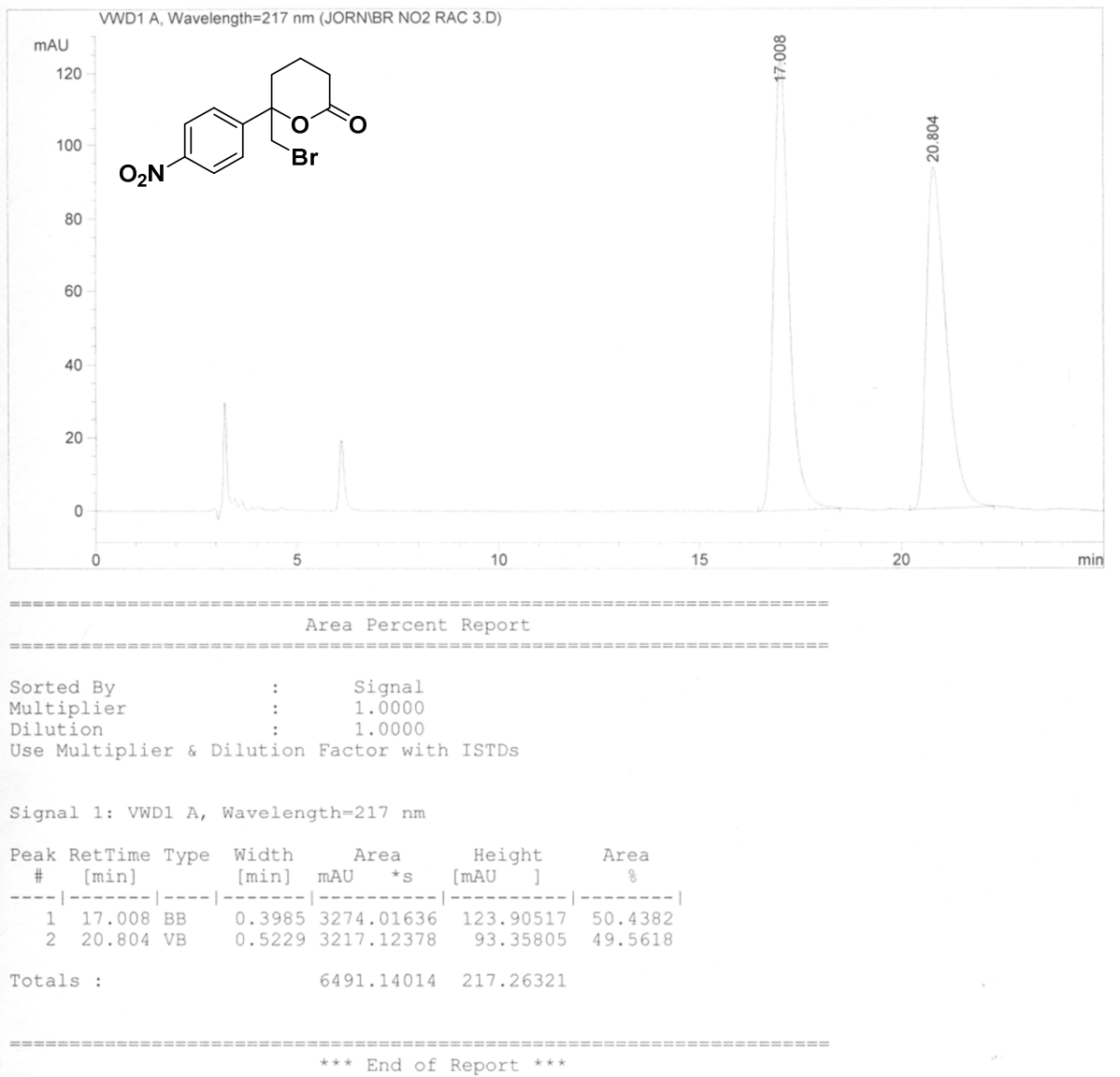

Figure S-82 HPLC chromatogram of racemic compound $\mathbf{6 j}$.

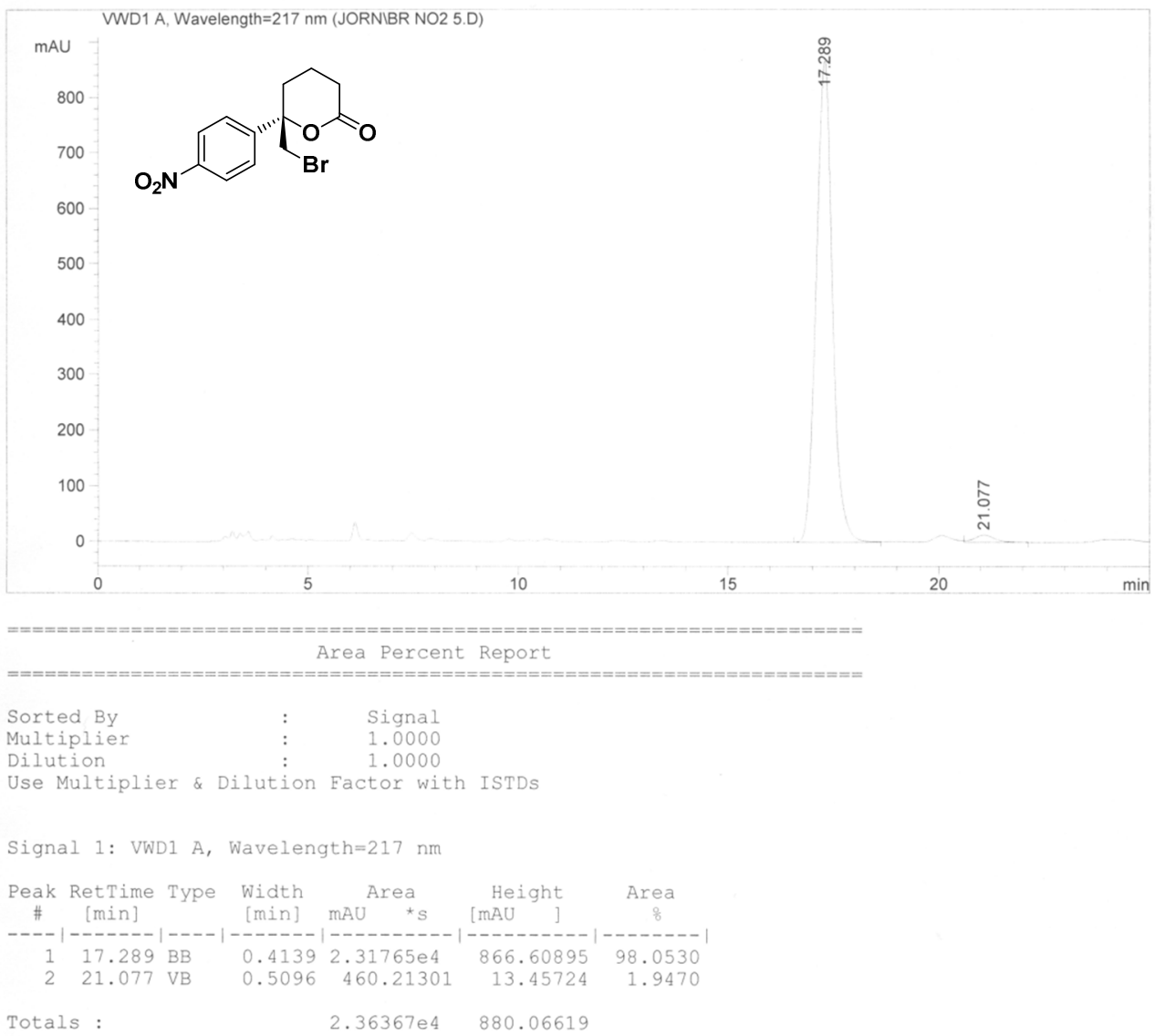

Figure S-83 HPLC chromatogram of compound $\mathbf{6 j}$. 


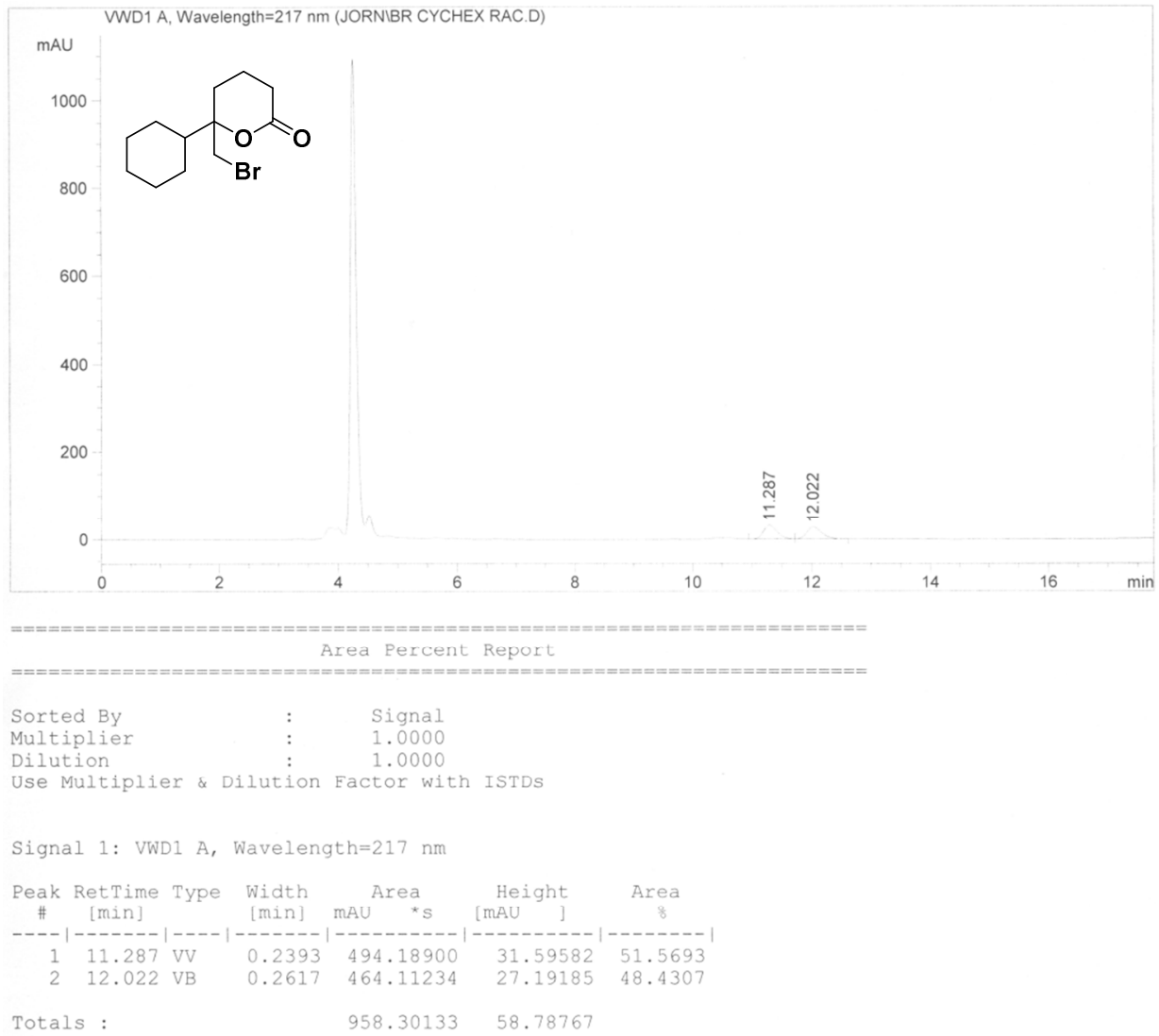

Figure S-84 HPLC chromatogram of racemic compound $6 \mathbf{k}$.

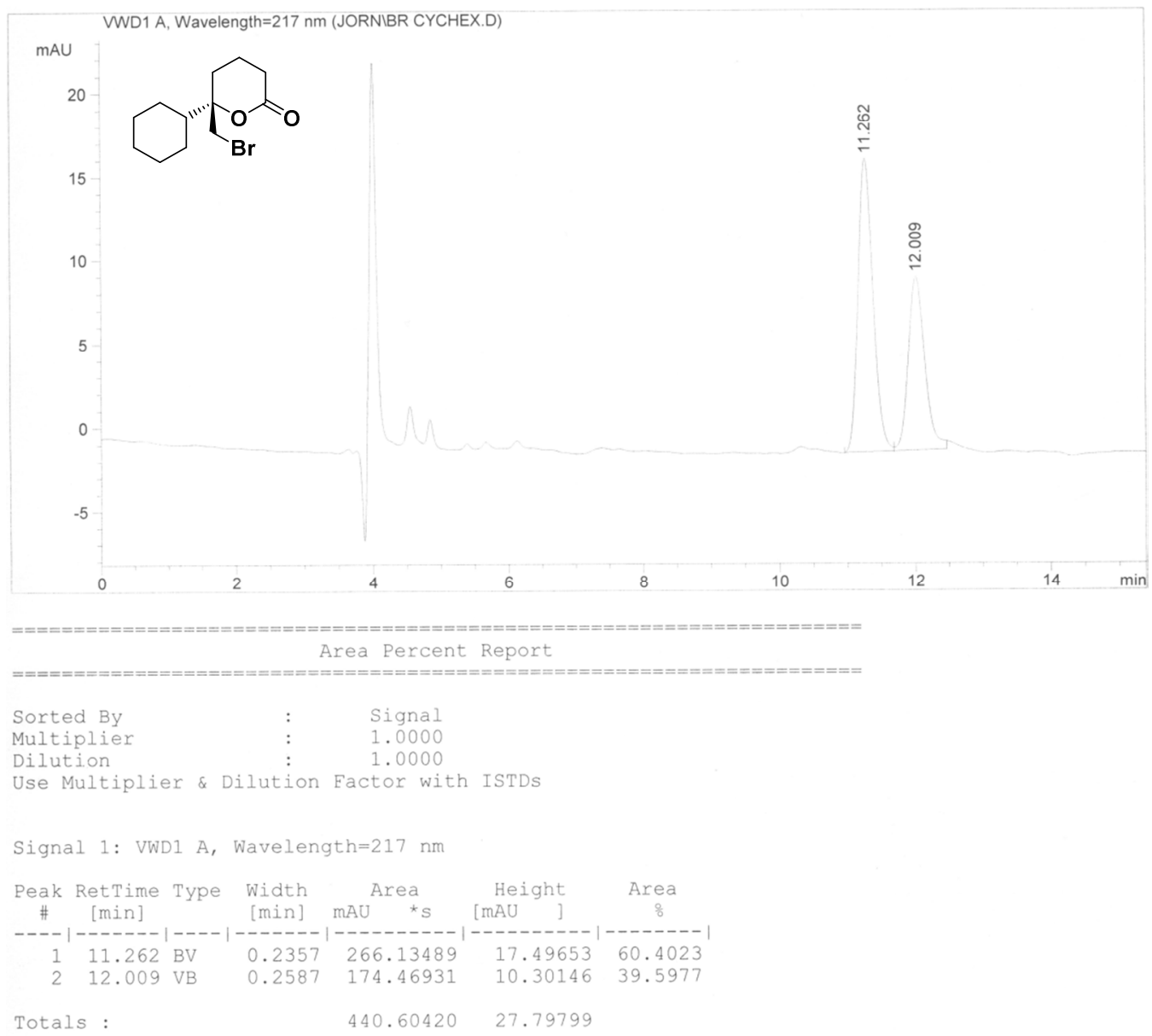

Figure S-85 HPLC chromatogram of compound $\mathbf{6 k}$. 


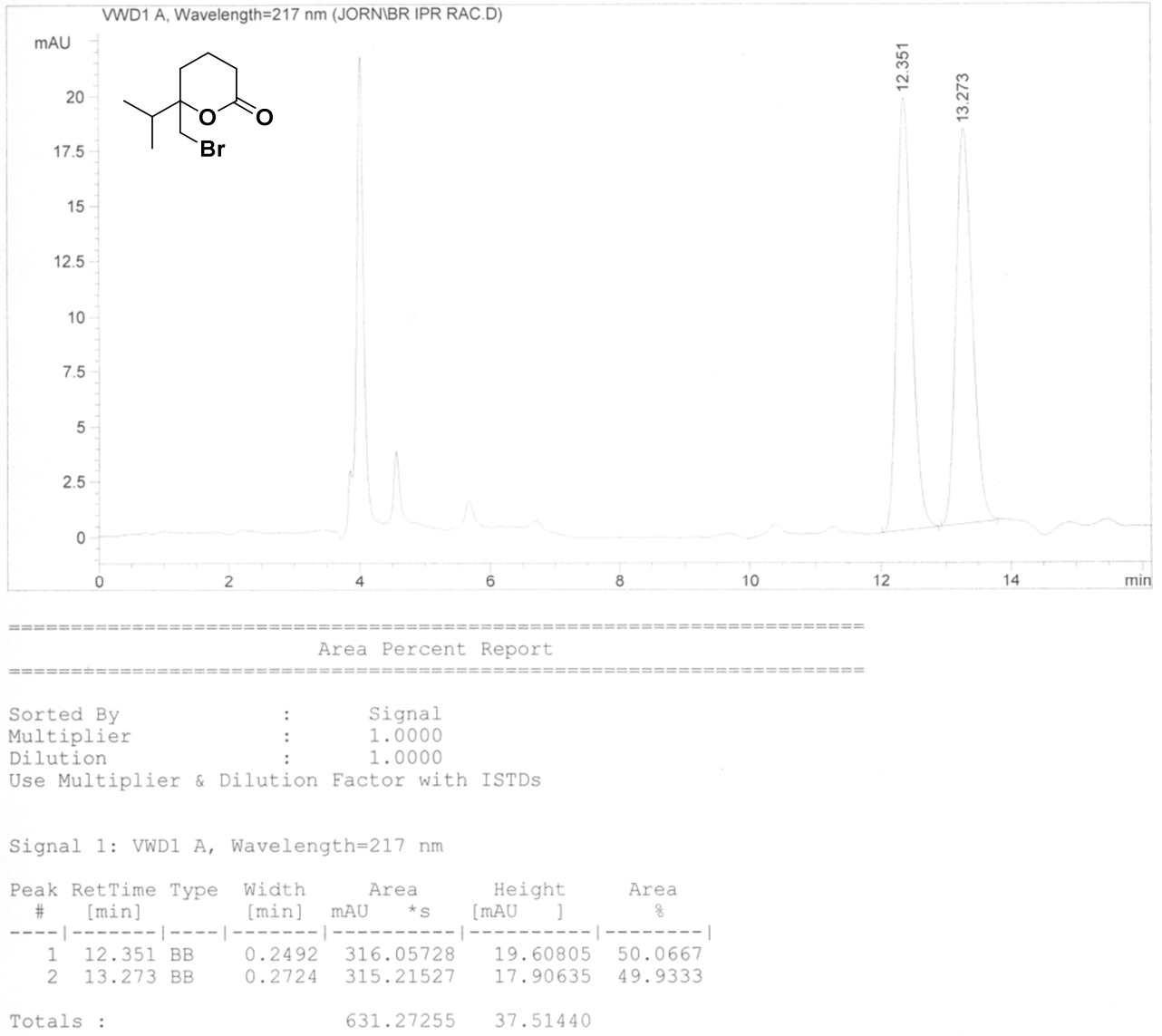

Figure S-86 HPLC chromatogram of racemic compound 61.

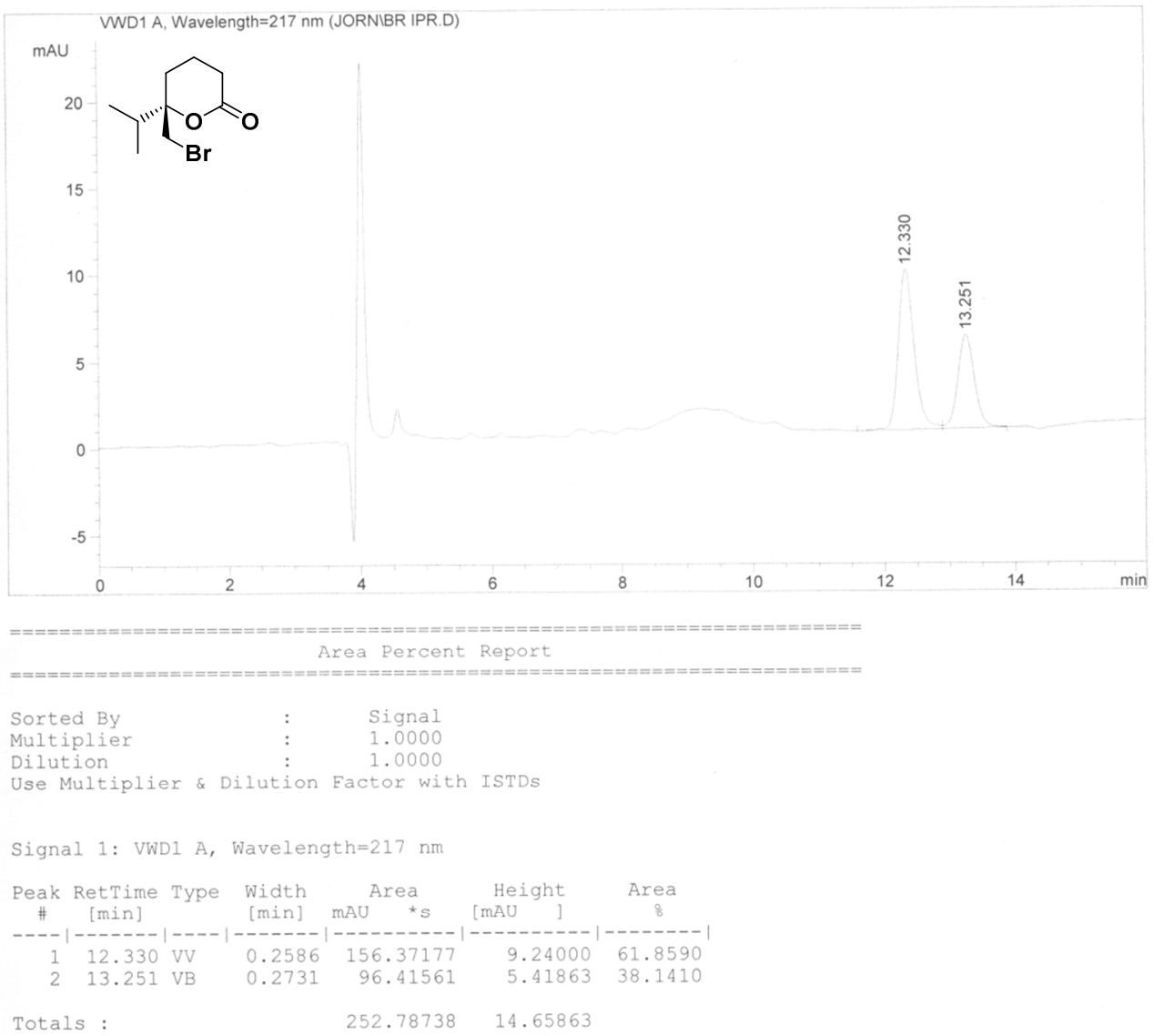

Figure S-87 HPLC chromatogram of compound $6 \mathbf{6}$. 


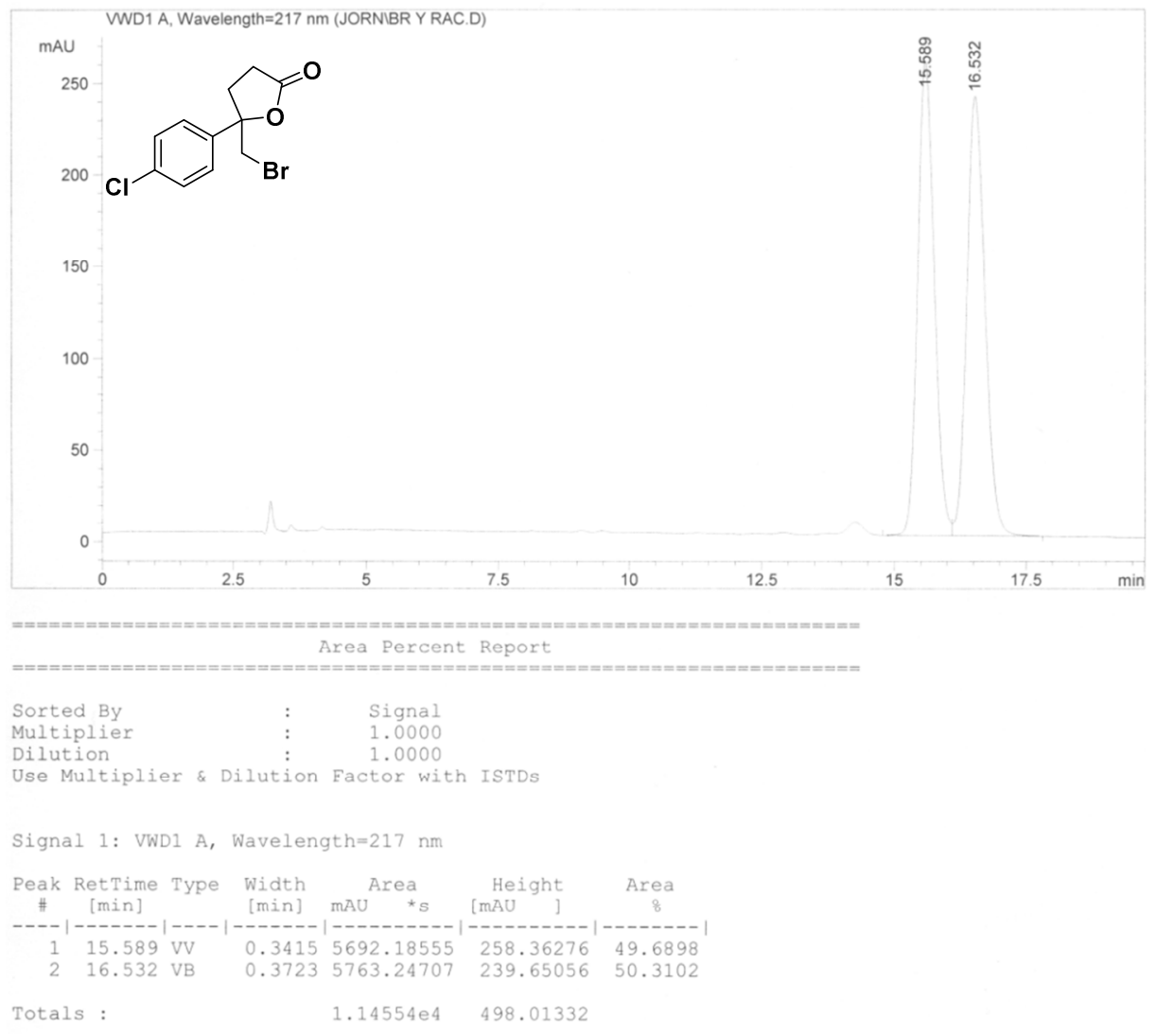

Figure S-88 HPLC chromatogram of compound racemic 5-(bromomethyl)-5-(4-chlorophenyl)dihydrofuran2(3H)-one.

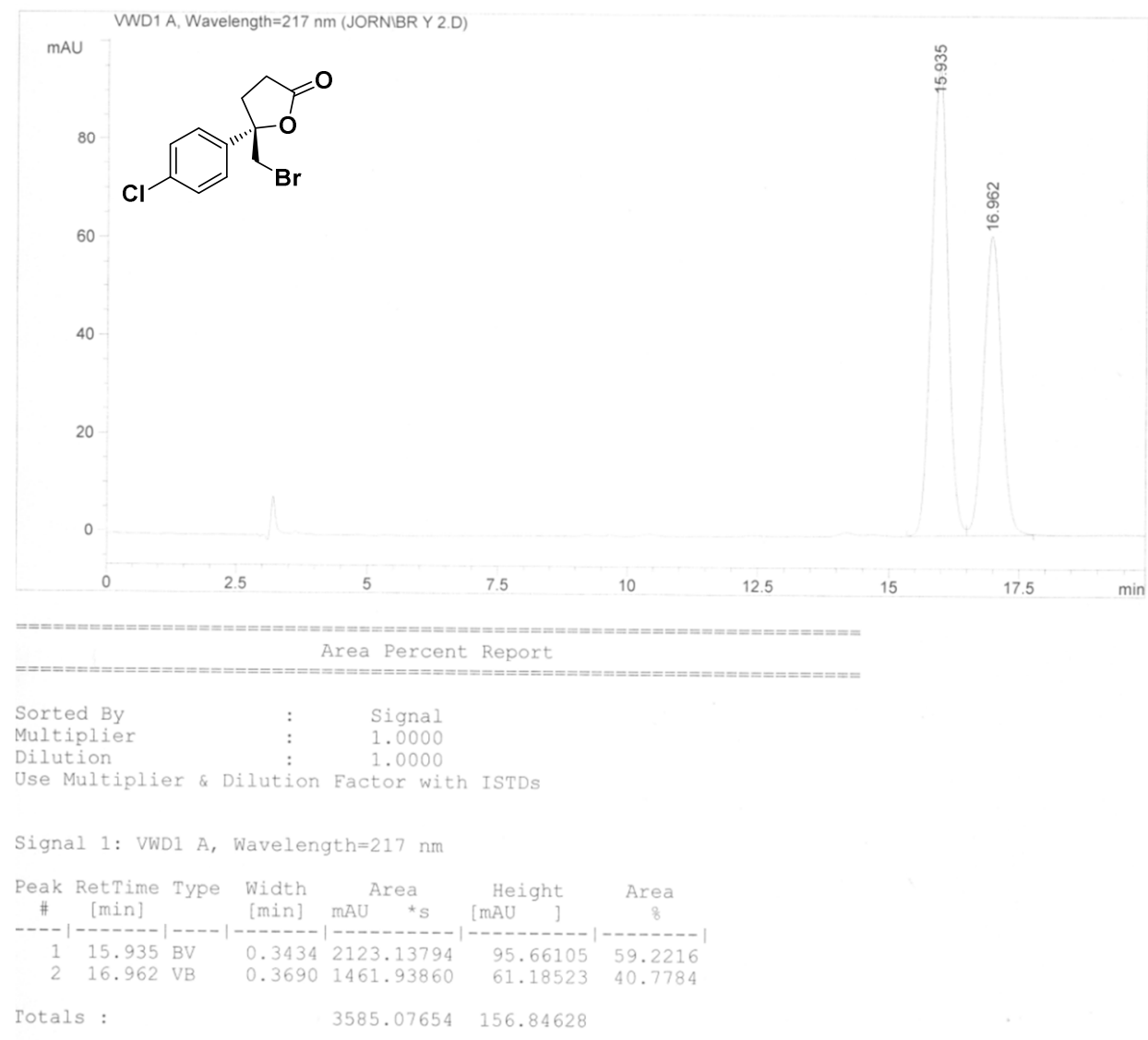

Figure S-89 HPLC chromatogram of compound $(S)$-5-(bromomethyl)-5-(4-chlorophenyl)dihydrofuran-2(3H)one. 


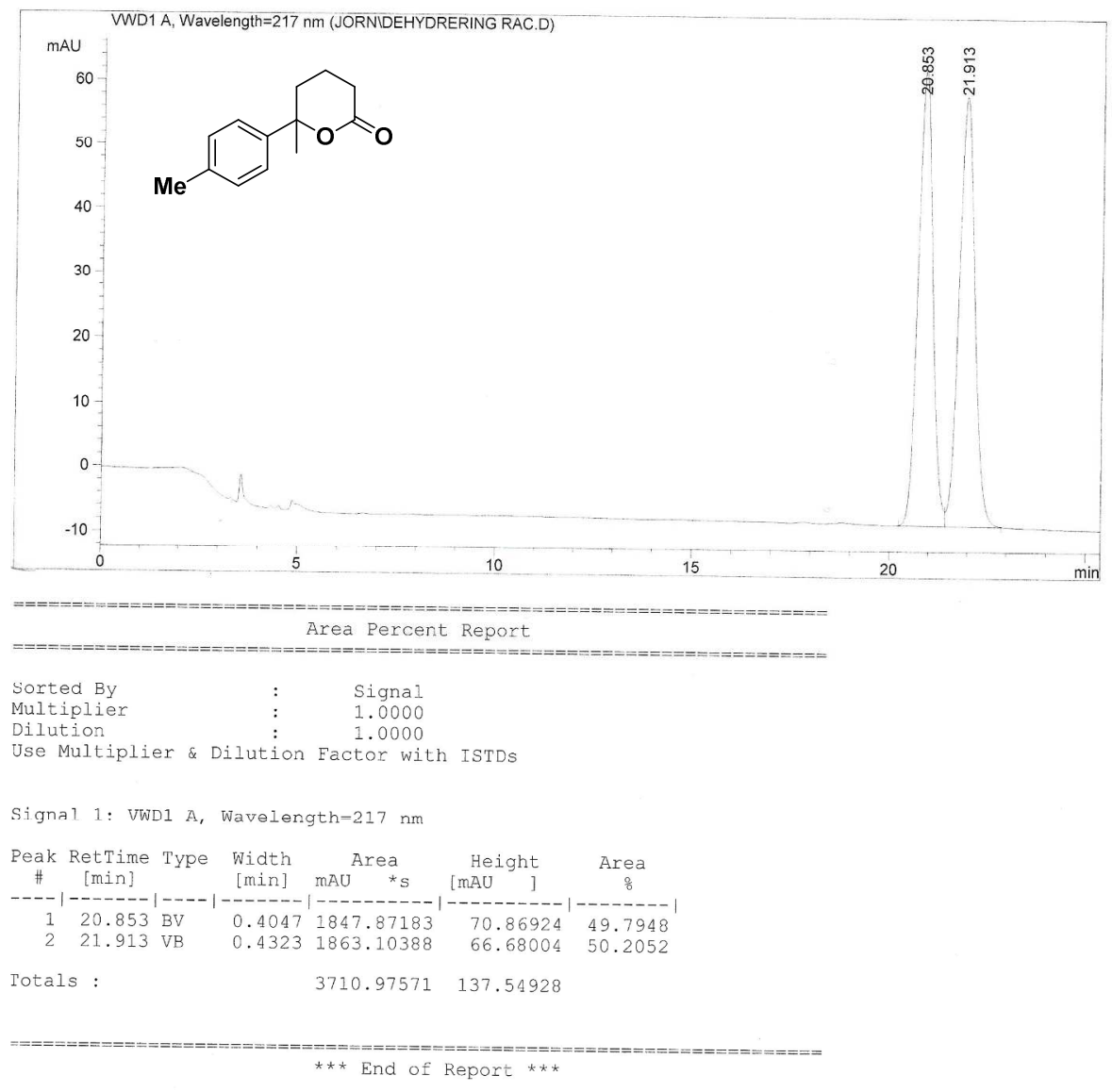

Figure S-90 HPLC chromatogram of racemic 12.

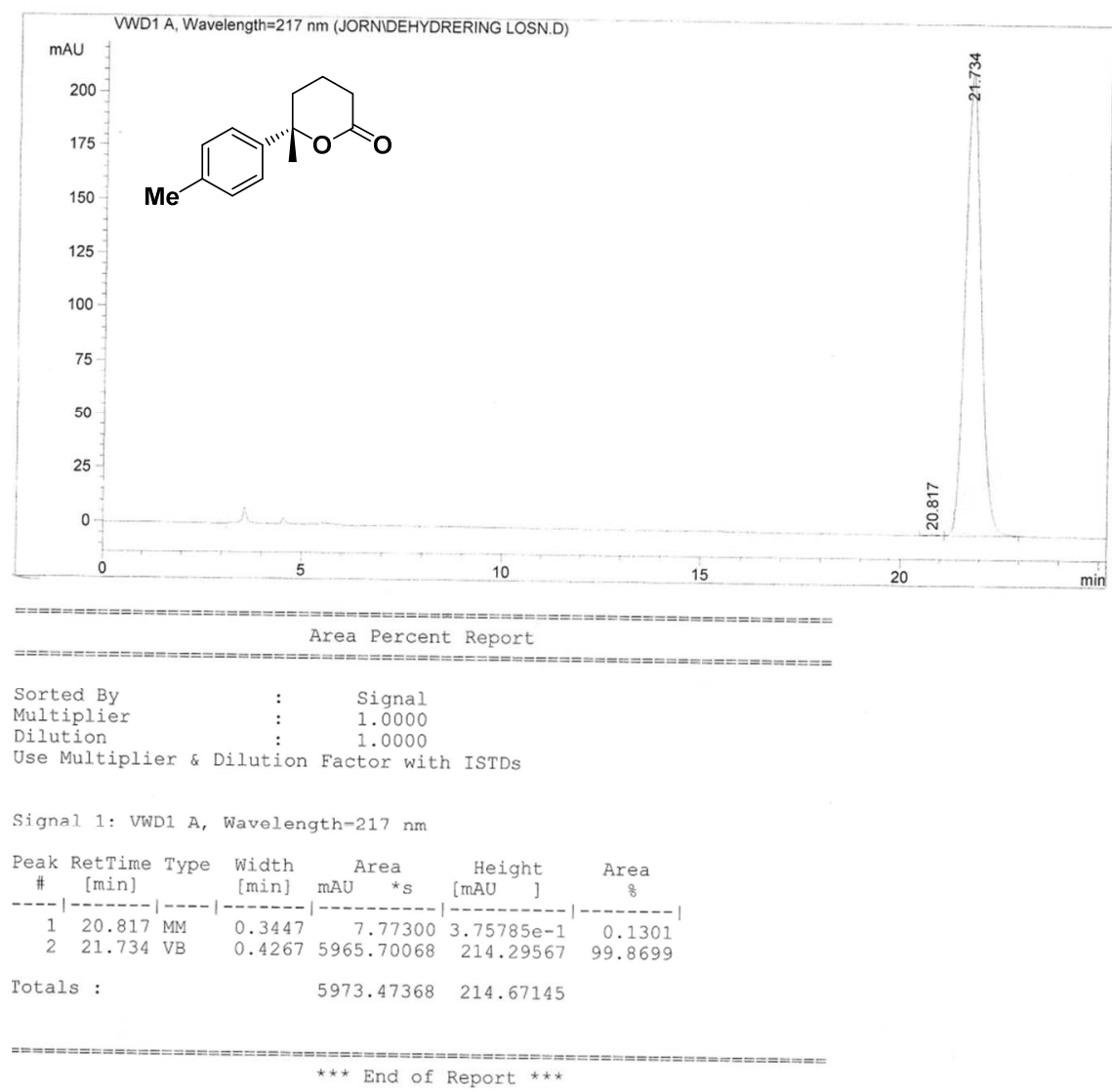

Figure S-91 HPLC chromatogram of recrystallized compound 12. 


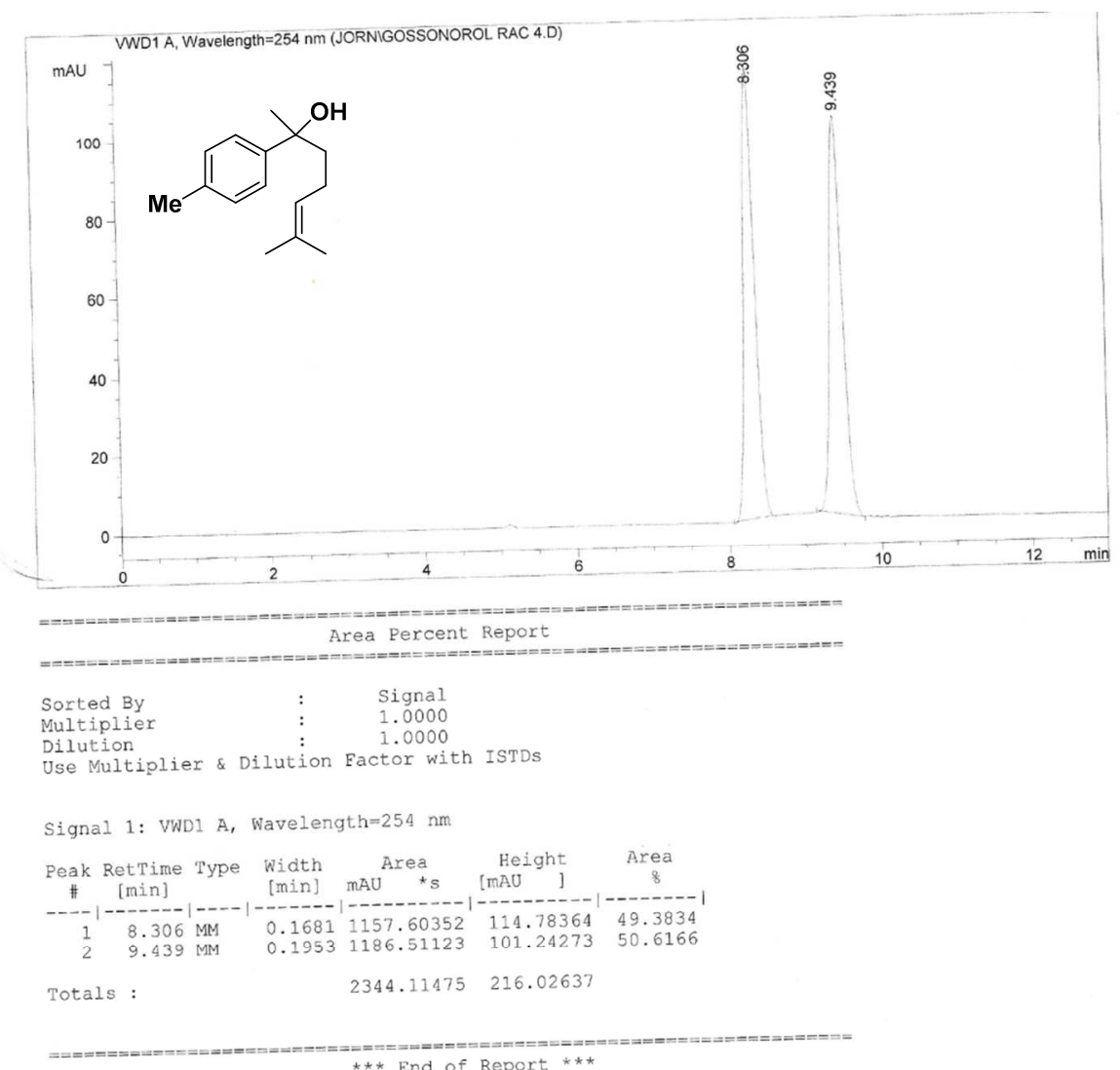

Figure S-92 HPLC chromatogram of ( \pm )-gossonorol (7).

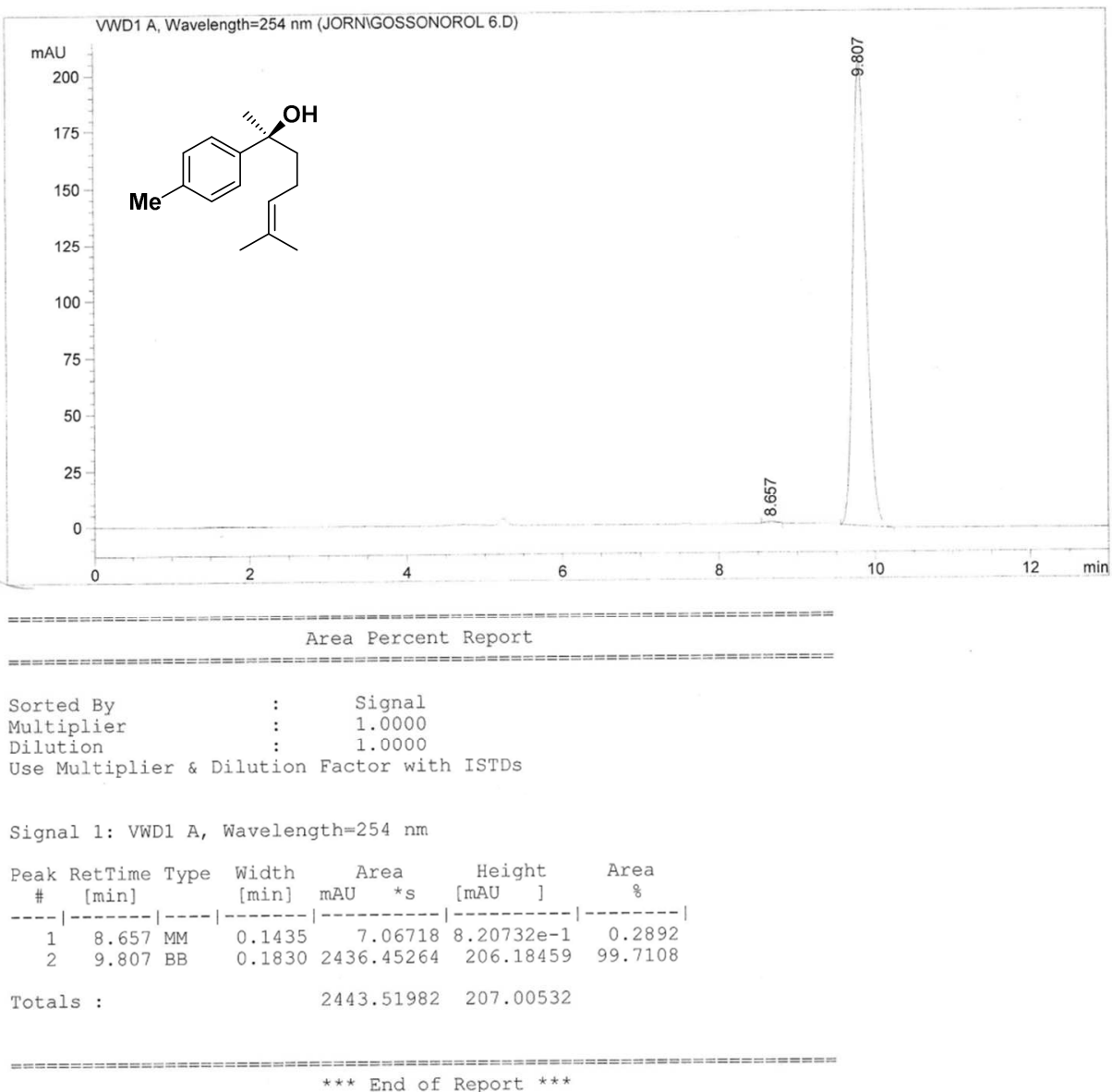

Figure S-93 HPLC chromatogram of (-)-gossonorol (7). 


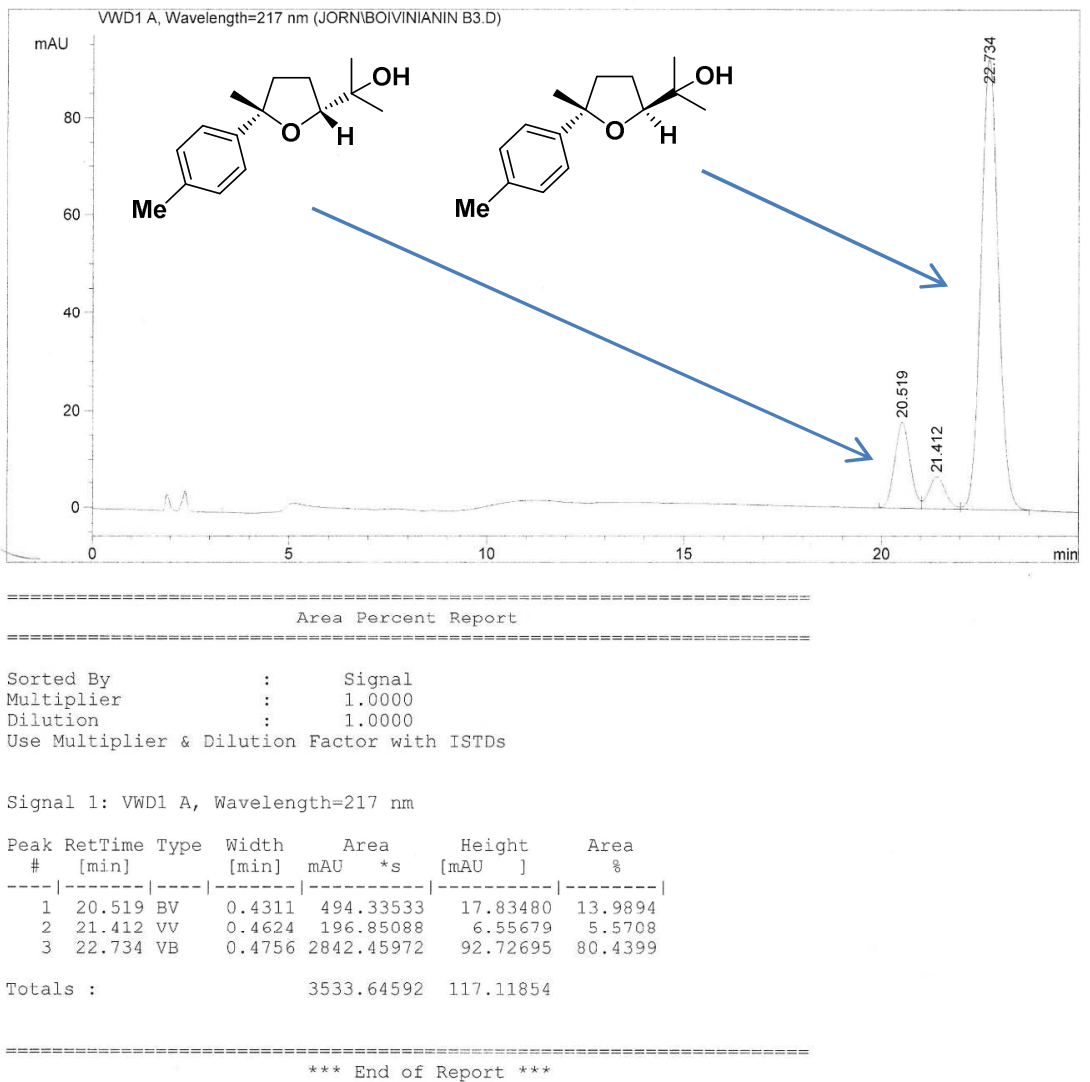

Figure S-94 HPLC chromatogram of crude boivinianin B (9).

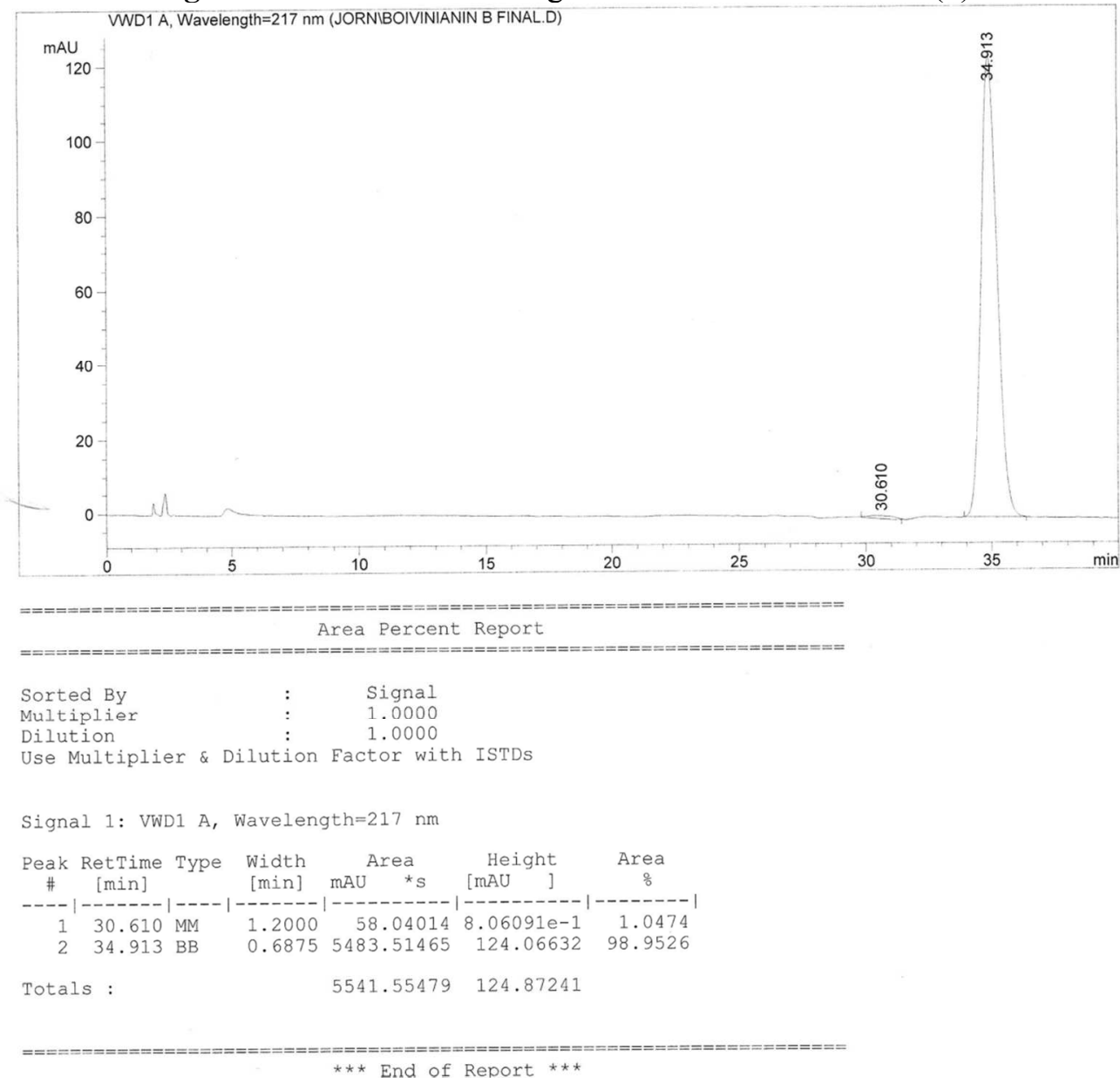

Figure S-95 HPLC chromatogram of (-)-boivinianin B (9). 


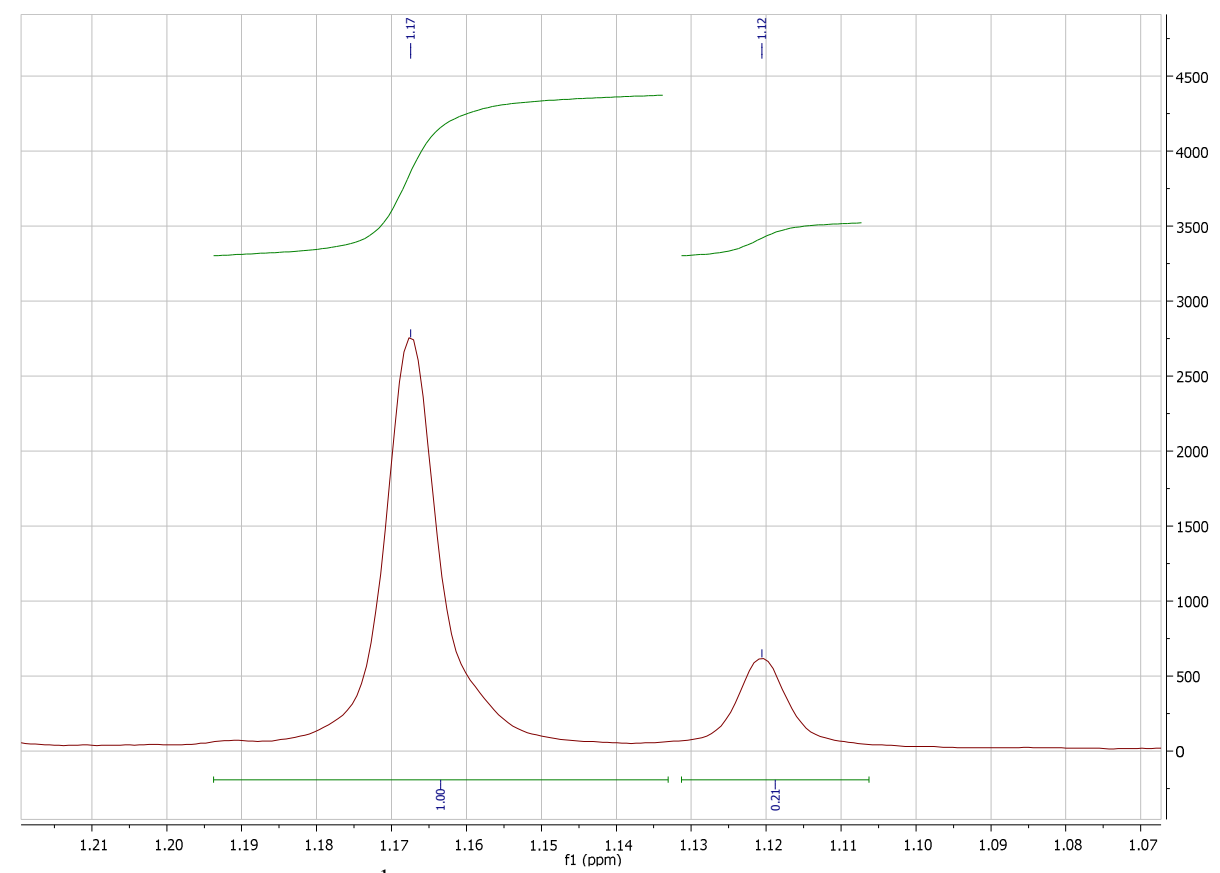

Figure S-96 ${ }^{1}$ H-NMR spectrum of crude boivinianin B (9).

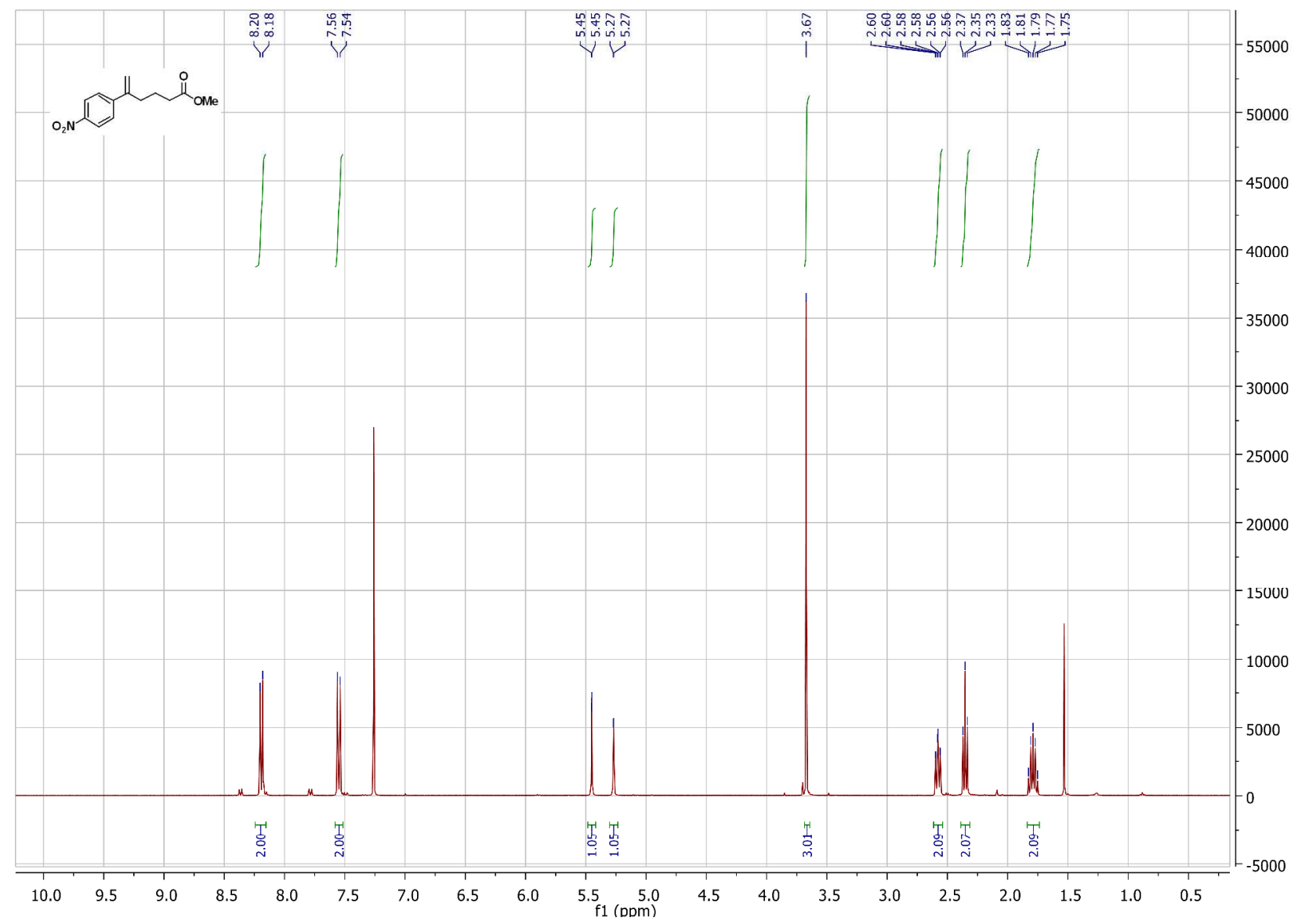

Figure S-97 ${ }^{1} \mathrm{H}-\mathrm{NMR}$ spectrum of Methyl 5-(4-nitrophenyl)hex-5-enoate (11j) 


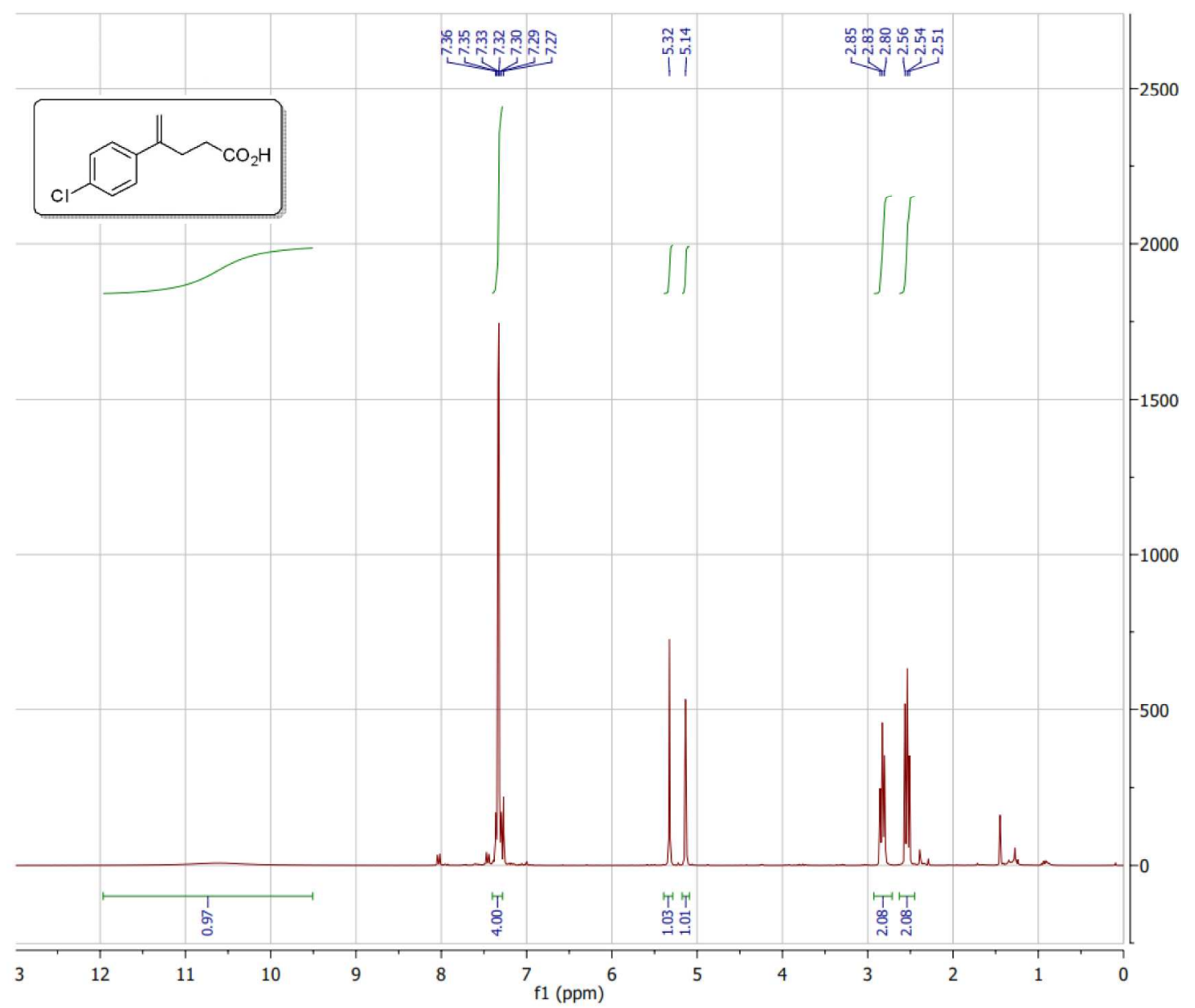

Figure S-98 ${ }^{1} \mathrm{H}-\mathrm{NMR}$ spectrum of 4-(4-Chlorophenyl)pent-4-enoic acid 


\section{References}

1. Tungen, J. E.; Nolsøe, J. M. J.; Hansen, T. V. Org. Lett. 2012,14, 5884.

2. Abecassis, K.; Gibson. S. E. Eur. J. Org. Chem. 2010, 15, 2938 